\title{
Raman Spectroscopy of Human Lens Epithelial Cells Exposed to a Low-dose Range of Ionizing Radiation
}

\author{
by \\ Harry Allen \\ A thesis submitted to the Faculty of Graduate and \\ for the degree of \\ Master of Science \\ in \\ Physics \\ Specialization in Medical Physics \\ Ottawa Carleton Institute for Physics \\ Carleton University \\ Ottawa, Ontario \\ (C) 2018
} Postdoctoral Affairs in partial fulfillment of the requirements

Harry Allen 


\begin{abstract}
Recent studies indicate that ionizing radiation induced opacification in the lens of the eye occurs at lower doses $(<2$ Gy) than past protection guidelines had assumed. Research is currently focused on identifying early signs of the lens degradation that leads to cataract formation, and in developing non-invasive assays capable of detecting low dose exposures to the lens of the eye. Raman spectroscopy (RS) is a non-invasive, vibrational spectroscopic technique based on the inelastic scattering of light by molecular vibrations. It is capable of providing information on the molecular makeup of biological samples that can be used for classification purposes. This work focuses on the application of RS combined with multivariate statistical analysis to detect radiation induced changes in vitro within human lens epithelial (HLE) cells exposed to a broad dose-range (0.01-5 Gy). The development of a new Raman microscope which will increase data acquisition throughput is also discussed.
\end{abstract}




\section{Acknowledgements}

I would like to begin by thanking all members of the Carleton Biophotonics Research Group (CBRG), both past and present. First and foremost, I would like to express my heartfelt gratitude to my supervisor, Dr. Sangeeta Murugkar, for providing invaluable advice and guidance, for keeping me on track throughout my work, and without whom this research would not have happened. I would like to thank my predecessor, Hamid Moradi, who, with the help of Abrar Ahmad and Dean Sheperdson, built the original instrument on which my research was performed, and who showed me every detail about how it works, how to align it, and how to use it. Hamid provided me a solid foundation of both knowledge and skill in this field for which I will always be grateful. Dr. Balazs Nyiri of the Ottawa Hospital is another member of the CBRG who provided invaluable advice and opinions critical to the experiment as a whole.

I would like to thank Abrar Ahmad, Christopher Dedek, and Achint Kumar, whose work was also instrumental in performing this experiment. Abrar wrote the analysis code on which I based my own and which I always used to cross-check results. Chris figured out how to automate the stage in coordination with the spectroscopic software, which simplified things immensely. Achint was my partner in data acquisition and initial analyses. I am grateful for his willingness to take the late shift

on most of our day long acquisitions. I am also grateful for the work done by new team member Ben Hansson, who determined which laser and optical fibers would be best for the new Raman microscope, and Aditya Babu, who assisted me in aligning and testing the new Raman microscope, as well as in building the new brightfield illumination path.

From Health Canada, I would like to sincerely thank Dr. Vinita Chauhan and Dr. Sami Qutob for their invaluable contributions. Dr. Chauhan conceived of the need for this work, and was responsible for growing, irradiating, and transporting 
the cells. Dr. Qutob provided the clonogenic assay data and helped with growing and transporting cells. I would also like to express my thanks to Barbara Kutzner for teaching us how to properly use the biosafety cabinet and other instruments, and to anyone from Health Canada whose contributions I may not be aware of. The institution has been a pleasure to work with. 


\section{Statement of originality}

This thesis was written in whole by the author. Those who contributed experimentally to the results covered herein are acknowledged, and all references to theoretical and methodological materials are properly cited. Copyright permission has been acquired for any images shown which were not the author's own work, and the copyright holder acknowledged in the relevant figure caption.

The HLE cells were grown and irradiated at Health Canada by Dr. Vinita Chauhan. Sami Qutob performed the clonogenic assays. Data acquisition of Raman spectra was carried out by the author and Achint Kumar. Stage automation code was based on code originally written by Christopher Dedek. Data analysis was primarily carried out by the author with classification code based on code originally written by Abrar Ahmad, and Dr. Sangeeta Murugkar writing some of the data fitting code. Dr. Murugkar supervised all work performed at Carleton University.

\section{Peer-review papers}

C. H. Allen, A. Kumar, S. Qutob, B. Nyiri, V. Chauhan, and S. Murugkar, Raman micro-spectroscopy analysis of human lens epithelial cells exposed to a low-dose-range of ionizing radiation, Physics in Medicine \& Biology 63, 025002 (2018).

- The results of this study are reported in chapter 5 


\section{Contents}

$\begin{array}{ll}\text { Abstract } & \text { i }\end{array}$

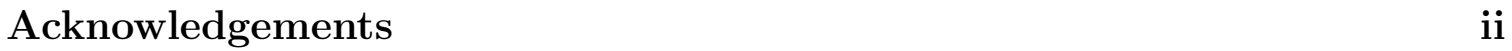

Statement of originality $\quad$ iv

Table of contents $\quad$ v

List of tables $\quad$ xi

List of figures $\quad$ XV

Nomenclature $\quad$ xvi

1 Introduction 1

1.1 Radiosensitivity of the lens of the eye . . . . . . . . . . 1

1.2 Raman spectroscopy as a label-free technique for biomedical research 2

1.2.1 Detecting and characterizing radiation induced changes using RS 4

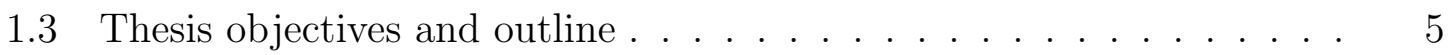




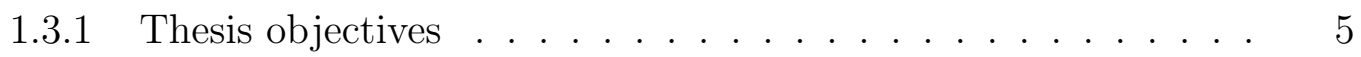

1.3 .2 Thesis outline $\ldots \ldots \ldots \ldots \ldots \ldots \ldots \ldots$

2 Ionizing Radiation and Radiobiology of HLE cells 8

2.1 Ionizing Radiation $\ldots \ldots \ldots \ldots \ldots \ldots$

2.1 .1 Types of ionizing radiation . . . . . . . . . . 8

2.1.2 Absorbed dose . . . . . . . . . . . . . . . . . . . . . 12

2.2 Biological effects of radiation exposure $\ldots \ldots \ldots \ldots \ldots$

2.2 .1 Cell composition and DNA . . . . . . . . . . . . . 14

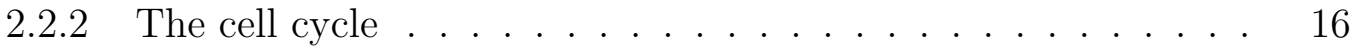

2.2.3 Radiation induced free radical formation in water . . . . . 16

2.2 .4 DNA damage $\ldots \ldots \ldots \ldots \ldots \ldots \ldots$

2.2 .5 Cellular response $\ldots \ldots \ldots \ldots \ldots$

2.2.6 Survival curves and the linear quadratic model of cell survival 20

2.2.7 Low-dose hypersensitivity and induced radioresistance . . . . 21

2.3 Human lens epithelial cells . . . . . . . . . . . . . . . . . . . . 23

3 Raman spectroscopy and multivariate statistical analysis $\quad 26$

3.1 Raman spectroscopy . . . . . . . . . . . . . . . . . . . 26

3.1 .1 Raman scattering . . . . . . . . . . . . . . . . . . . 26

3.1 .2 Molecular vibrations . . . . . . . . . . . . . . . . . 30

3.1.3 Raman shift and Raman spectra . . . . . . . . . . . . . 33

3.1 .4 Spectrometer . . . . . . . . . . . . . . 36 
3.1.5 Diffraction gratings . . . . . . . . . . . . . . . . 36

3.1.6 Deep depletion CCD . . . . . . . . . . . . . . . . 38

3.1.7 Long-pass dichroic mirror _. . . . . . . . . . . . . . . . . 40

3.1.8 Microscope objective . . . . . . . . . . . . . . . . . . . 40

3.2 Multivariate statistical techniques . . . . . . . . . . . . . . . 42

3.2.1 Linear algebra and data representation . . . . . . . . . . . 43

3.2.2 Principal component analysis . . . . . . . . . . . . . . 44

3.2.3 Linear Discriminant Analysis . . . . . . . . . . . . . . . 47

3.2.4 Cross-validation . . . . . . . . . . . . . . . . 50

\section{Materials and methods $\quad 53$}

4.1 Carleton Raman Microscope . . . . . . . . . . . . . . . . . 53

$4.1 .1 \quad$ Laser . . . . . . . . . . . . . . . . . . . . 56

4.1.2 Andor spectrometer . . . . . . . . . . . . . 57

4.2 System performance . . . . . . . . . . . . . . . 58

4.2.1 Silicon and Polystyrene . . . . . . . . . . . . . . 59

4.3 Sample preparation . . . . . . . . . . . . . . . . . . 59

4.3.1 Seeding HLE cells . . . . . . . . . . . . . . . . . . . . . . . . . 60

4.3.2 Irradiating cells . . . . . . . . . . . . . . . . . . . . . 60

4.4 Data acquisition . . . . . . . . . . . . . . . . 61

4.5 Data preprocessing . . . . . . . . . . . . . . . 62

4.5.1 Background subtraction .............. 63 
4.5.2 Spectral smoothing ................. 64

4.5.3 Vector Normalization . . . . . . . . . . . . . . . 65

4.6 Data analysis ....................... 65

4.6.1 Welch's t-test . . . . . . . . . . . . . 66

4.6 .2 PCA-LDA ....................... 67

4.6 .3 Curve fitting. . . . . . . . . . . . . . . 69

5 Results \& Discussion $\quad 71$

5.1 Acquisition of Raman Spectra from Nucleus and Cytoplasm . . . . . 71

5.1.1 Brightfield images of HLE cells . . . . . . . . . . . . 71

5.1 .2 Nucleus spectra . . . . . . . . . . . . . . . . . 72

5.1.3 Cytoplasm Spectra . . . . . . . . . . . . . . . 77

5.2 Differences between mean dose and mean control spectra . . . . . . . 80

5.3 PCA-LDA results . . . . . . . . . . . . . . . 85

5.3.1 Principal Component Analysis . . . . . . . . . . . 85

5.3.2 Contribution of cell cycle to spectra variance . . . . . . . . 86

5.3.3 Binary classification between each dose and control . . . . . . 87

5.3.4 Analysis of Linear Discriminants and Molecular Assignments . 89

5.4 Ultra-low dose response . . . . . . . . . . . . . . . . . . . . . 97

6 Instrumentation upgrade $\quad 101$

6.1 Limitations of previous apparatus . . . . . . . . . . . . . 101

6.1.1 Spot size and grid measurements . . . . . . . . . . . . . 102 
6.1.2 Laser power . . . . . . . . . . . . . . . . . . . . . 103

6.1.3 Confocality losses . . . . . . . . . . . . . . . . 104

6.1.4 Brightfield imaging . . . . . . . . . . . . . . . 104

6.1.5 Modularity ..................... 105

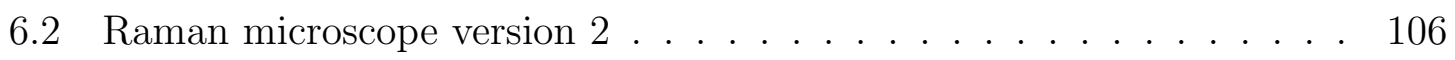

6.2.1 High power laser ... . . . . . . . . . . . . . 109

6.2.2 Fiber coupling. . . . . . . . . . . . . . 109

6.2.3 Improved brightfield imaging . . . . . . . . . . . . . . . 111

6.3 Performance . . . . . . . . . . . . . . . . . . 112

6.3.1 Silicon and polystyrene . . . . . . . . . . . . . 114

$\begin{array}{lll}7 & \text { Conclusions and future work } & 117\end{array}$

7.1 Conclusions ......................... 117

7.2 Future work . . . . . . . . . . . . . . . . 118

$\begin{array}{lr}\text { A Derivations } & 121\end{array}$

A.1 Normal Coordinates . . . . . . . . . . . . . . . . . 121

A.2 Mean and variance of LD scores . . . . . . . . . . . . . . . . . 124

A.3 Variance of LOOCV accuracy . . . . . . . . . . . . . . . . . . . 124

A.4 PCA using SVD . . . . . . . . . . . . . . . . 125

B Protocol for acquiring Raman spectra from HLE samples $\quad 127$

$\begin{array}{ll}\text { C Additional Figures } & 130\end{array}$ 
C.1 Cell cycle analysis results . . . . . . . . . . . . . . . . . . . 130

C.2 Photograph of Carleton Raman Microscope versions 1 and 2 . . . . . 132

C.3 Plots of accuracy vs number of PCs . . . . . . . . . . . . . . . 132

C.3.1 Nucleus ........................ 133

C.3.2 Cytoplasm ......................... 135

$\begin{array}{lr}\text { References } & 146\end{array}$ 


\section{List of Tables}

5.1 Peak assignments for prominent peaks of mean control nucleus spectrum 76

5.2 Peak assignments for prominent peaks of mean control cytoplasm spec-

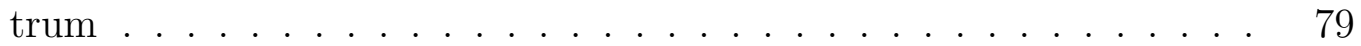

5.3 Binary classification LOOCV sensitivity, specificity, and accuracy results 87

5.4 Tentative Raman band assignments for LD Loadings from nucleus . . 95

5.5 Tentative Raman band assignments for LD Loadings from cytoplasm 96 


\section{List of Figures}

2.1 Mass attenuation coefficients for water as a function of photon energy 11

2.2 Absorbed dose in a small volume . . . . . . . . . . . . . . . 13

2.3 Linear quadratic model of cell survival for different $\alpha / \beta$ values . . . . 21

2.4 Diagram of human eye, showing lens position relative to cornea and

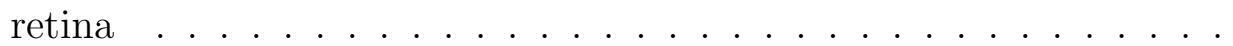

2.5 Diagram of human lens, showing fiber structure and location of epithe-

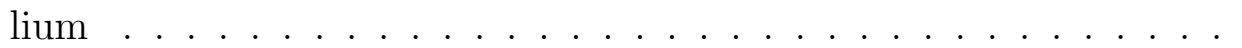

3.1 Changes in energy level of scattering molecule and scattered photon. .

3.2 Carbon dioxide $\mathrm{CO}_{2}$ vibrational modes . . . . . . . . . . . . . . . . 32

3.3 Benzene $\left(\mathrm{C}_{6} \mathrm{H}_{6}\right)$ group vibrational modes . . . . . . . . . . . .

3.4 Examples of Raman spectra from various materials . . . . . . . . .

3.5 layout of main optical components in a Czerny-Turner Raman spec-

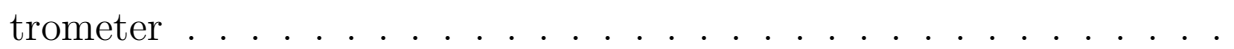

3.6 Illustration of blazed grating showing reflection angles . . . . . . . . . 37

3.7 Microscope objective focusing on a point P directly below the pupil. . 41

3.8 Two-dimensional PCA visualization . . . . . . . . . . . . . . . 46 
3.9 Comparison between high and low PCs . . . . . . . . . . . . 47

3.10 LDA visualization in two dimensions . . . . . . . . . . . . . . . . 48

4.1 Schematic of the Carleton Raman Microscope version 1 . . . . . . . 55

4.2 How a pinhole provides confocality . . . . . . . . . . . . 56

4.3 Background subtraction using SMIRF . . . . . . . . . . . . . 64

4.4 Plot of LOOCV accuracy vs number of PCs . . . . . . . . . . 69

5.1 Brightfield image of HLE cells with 3x3 point grid shown over nucleus 72

5.2 Plot of spectral intensity vs distance above quartz surface . . . . . . . 73

5.3 Individual nucleus point spectra from a 3x3 point grid . . . . . . . . . 74

5.4 Mean of 9 point spectra for an individual nucleus . . . . . . . . . . 75

5.5 Mean Raman spectrum for nucleus using all 68 control spectra . . . . 75

5.6 Individual cytoplasm point spectra . . . . . . . . . . . . . . . 77

5.7 Mean of 9 cytoplasm point spectra . . . . . . . . . . . . 78

5.8 Mean Raman spectrum for cytoplasm using all 64 control spectra . . 78

5.9 Mean nucleus spectra for all doses . . . . . . . . . . . . . . . 81

5.10 Close-up of sections of mean nucleus spectra for all doses . . . . . . . 82

5.11 Mean cytoplasm spectra for all doses . . . . . . . . . . . 83

5.12 Close-up of sections of mean cytoplasm spectra for all doses . . . . . 84

5.13 First 4 PCs for each nucleus dose-control pair . . . . . . . . . . . 85

5.14 First 4 PCs for each cytoplasm dose-control pair . . . . . . . . . . . . 86

5.15 Nucleus LOOCV LD score distributions . . . . . . . . . . . . . 88 
5.16 Cytoplasm LOOCV LD score distributions . . . . . . . . . . . . . 89

5.17 Nucleus LDs and difference spectra (0.25 Gy, 0.5 Gy) . . . . . . . . 90

5.18 Nucleus LDs and difference spectra (2 Gy, 5 Gy) . . . . . . . . . . 91

5.19 Cytoplasm LDs and difference spectra (0.25 Gy, 0.25 Gy) . . . . . . . 92

5.20 Cytoplasm LDs and difference spectra (2 Gy, 5 Gy) . . . . . . . . 93

5.21 Raman band area absolute difference ratios fitted to induced repair

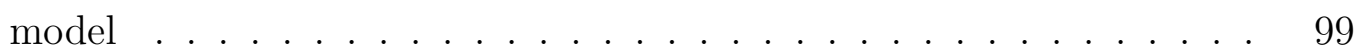

5.22 Clonogenic assay data $(\mathrm{n}=3)$ fitted to induced repair model . . . . . . 100

6.1 Schematic of newly built Carleton Raman Microscope version 2 . . . 107

6.2 fiber core layout for input end of bundled optical fiber. . . . . . . . . 110

6.3 fiber core layout for output end of bundled optical fiber. . . . . . . . 111

6.4 Peak intensity at $1001.4 \mathrm{~cm}^{-1}$ for polystyrene vs depth along z-axis. . 113

6.5 Plot of axial distribution of laser power in focal volume . . . . . . . . 114

6.6 Silicon spectra for CRM1 and CRM2 . . . . . . . . . . . . . . 115

6.7 Polystyrene spectra for CRM1 and CRM2 . . . . . . . . . . . . 116

C.1 Cell cycle percentages for 0 and 5 Gy . . . . . . . . . . . . 130

C.2 Photograph of the the Carleton Raman Microscope . . . . . . . . . . 131

C.3 Photograph of Carleton Raman microscope version 2 . . . . . . . . . 132

C.4 LOOCV accuracy vs number of PCs used for nucleus, 0 vs 0.25 Gy . 133

C.5 LOOCV accuracy vs number of PCs used for nucleus, 0 vs 0.5 Gy . . 133

C.6 LOOCV accuracy vs number of PCs used for nucleus, 0 vs 2 Gy . . . 134 
C.7 LOOCV accuracy vs number of PCs used for nucleus, 0 vs 5 Gy . . . 134

C.8 LOOCV accuracy vs number of PCs used for cytoplasm, 0 vs 0.25 Gy 135

C.9 LOOCV accuracy vs number of PCs used for cytoplasm, 0 vs 0.5 Gy 135

C.10 LOOCV accuracy vs number of PCs used for cytoplasm, 0 vs 2 Gy 136

C.11 LOOCV accuracy vs number of PCs used for cytoplasm, 0 vs 5 Gy 136 


\section{Nomenclature}

$\begin{array}{ll}\text { CRM } & \text { Carleton Raman Microscope } \\ \text { DM } & \text { Dichroic mirror } \\ \text { HLE } & \text { Human lens epithelial } \\ \text { LD } & \text { Linear discriminant } \\ \text { LDA } & \text { Linear discriminant analysis } \\ \text { LE } & \text { Lens epithelial } \\ \text { LET } & \text { Linear energy transfer } \\ \text { LF } & \text { Lens fiber } \\ \text { LOOCV } & \text { Leave-one-out cross-validation } \\ \text { NA } & \text { Numerical aperture } \\ \text { NIR } & \text { Near-infrared } \\ \text { PC } & \text { Principal component } \\ \text { PCA } & \text { Principal component analysis } \\ \text { RS } & \text { Raman scattering } \\ \text { SVD } & \text { Singular value decomposition }\end{array}$




\section{Chapter 1}

\section{Introduction}

\subsection{Radiosensitivity of the lens of the eye}

Ionizing radiation has been a known medical hazard since shortly after its discovery, when Henri Becquerel discovered a burn on his skin beneath his vest pocket, in which he had kept a sample of radium, and which Pierre Curie reproduced in 1901 by producing a similar burn on his forearm. ${ }^{1}$ In modern times, new medical procedures that make use of ionizing radiation for real-time diagnostic and operational guidance imaging, such as fluoroscopy, have led to an increase in reported eye injuries. ${ }^{2-4}$ The lens of the eye has been known to be radiosensitive for some time, as epidemiological studies in the past have demonstrated that acute doses of radiation lead to formation of cataracts, ${ }^{1,5}$ and later studies have shown that the accumulated dose, for low doses $(\leq 0.5 \mathrm{GY})$ accumulated over years, increases the chance of cataract formation as well. ${ }^{3,6-8}$ In fact, the threshold dose estimate for cataractogensis was recently revised downward by the International Commission on Radiation Protection (ICRP) from $2 \mathrm{~Gy}$ to $0.5 \mathrm{~Gy} .{ }^{3}$ Most previous studies on the effects of irradiating the lens have focused on animal models at doses greater than $5 \mathrm{~Gy} .{ }^{5}$ 
The biological processes that lead to formation of opacities in the lens of the eye, and how exactly these processes are induced by exposure to IR, are not fully understood. Furthermore, there are no dosimetric methods that can estimate dose received to the lens specifically and to high precision. While modifications to current dosimetric assays could be imagined, those assays are invasive and time consuming. Thus, there is a desire for techniques that can be used to investigate radiation induced biochemical changes in the lens, as well dosimetric assays that are both non-invasive and quick. Raman spectroscopy (RS), is a label-free, non-invasive vibrational spectrographic technique capable of detecting changes in molecular structure and concentration in a given sample. It has long been used for investigating biological changes in cells, and over the past decade has been shown capable of detecting radiation induced changes in certain cell lines as well. For this reason, it is a leading candidate to solve both investigative and diagnostic desires.

\subsection{Raman spectroscopy as a label-free technique for biomedical research}

Raman spectroscopy $(\mathrm{RS})$ uses the inelastic scattering of light to probe the molecular makeup of a sample. A monochromatic laser, in either visible or infrared spectrum, is used as the source of incident photons. For spontaneous Raman scattering, in which the inelastic scattering occurs spontaneously and with no external stimulation, the scattering event is almost always of the Stokes variety, in which the photon loses some energy. This energy is transferred into a vibrational mode of the molecule. Typically, the chance of such a scatter is approximately 1 in $10^{-7}$, but this is sufficient to get a useful signal when using a laser of sufficient power.

The spectrum generated by the Raman scattered light then provides a molecular 
fingerprint, due to the set of vibrational states tending to be unique between different types of molecules. This allows for the detection of minute changes in the molecular composition of a sample without damaging it in any way. Being label-free, the sample remains unchanged and no chemical processing is required. For this reason, $\mathrm{RS}$ is widely used in areas where non-destructive sampling of molecular composition is desired. It is used in industries ranging from solar panel manufacturing, to the pharmaceutical industry. In research settings, it is widely used in departments of chemistry and biology for non-destructively measuring concentrations and structural forms of various molecules. Raman spectrometers equipped with lasers for use in specific roles are thus commodity items with several manufacturers such as Ocean Optics , Tornado Spectral Systems, Thermo Fisher Scientific, and many others. Raman microscopes for use in research, particularly biomedical research, are less common and far more expensive.

Raman spectroscopy has been used in biomedical research applications for years. It has been used to analyze individual cells, tissue samples, and various biofluids. ${ }^{9-11}$ Raman spectra in the near-infrared (NIR) range of 780-2500 nm are typically used in these applications because the fingerprint region, that is the region in wavelength in which characteristic Raman peaks appear in the spectrum, falls within this range. Combined with multivariate statistical methods for classification, RS has been used to distinguish between cisplatin-sensitive and cisplatin-resistant human ovarian cancer cells in vitro. ${ }^{12}$ It has similarly been used to distinguish between healthy and cancerous tissue in the brain and epidermis in vivo. ${ }^{13,14} \mathrm{RS}$ is thus well demonstrated as a useful tool in a wide range of biomedical applications. 


\subsubsection{Detecting and characterizing radiation induced changes using RS}

RS has been used to detect changes in cells and tissues exposed to ionizing radiation. ${ }^{15-25}$ Past studies on pure samples of concentrated DNA have shown radiation induced changes to DNA, ${ }^{26,27}$ believed to be due to structural changes. Similar studies have shown detectable changes in proteins, and lipids. ${ }^{28}$ Tissue studies from the last decade showed detectable spectral changes in irradiated mouse brain tissue, ${ }^{15}$ as well as in proton irradiated tissue samples. ${ }^{16}$ Characterizing the radio-sensitivity of oral cancer cells has also been investigated using RS. ${ }^{20}$

Studies relating to radiotherapy, such as investigating the effects of doses in the range 15-50 Gy using RS have been carried out on lung, breast, and kidney cancer cell lines, performed in vitro. ${ }^{17,18}$ Similar studies in a lower, clinically relevant dose range from 2-10 Gy have also been performed. ${ }^{21}$ One biomolecular change some of these studies detected was an increase of glycogen within radioresistant human lung cancer cells in vitro, ${ }^{22}$ as well as in vivo using tumour xenographs of the same cells. ${ }^{24}$

While these studies show great potential for using RS to detect biochemical changes in irradiated cells, they do not involve using RS for purposes of radiation dosimetry. Work done by Maguire et $a l^{23}$ showed it is possible to distinguish between spectra of irradiated and non-irradiated isolated and fixed lymphocytes at doses of 0.5 and 0.05 Gy, demonstrating sensitivity of RS to changes occurring at low doses. Another study, by Meade et $a l^{25}$ that involved using RS to detect changes in vitro in human keratinocytes irradiated at the same doses (0.5 and $0.05 \mathrm{~Gy}$ ), was able to discriminate between each dose and control to accuracies of $78 \%$ and $100 \%$ respectively. This study also involved irradiating cells over a broader dose range: 5, 20, 50, 200, 500, and $750 \mathrm{mGy}$, as well as 1, 2.5, and 5 Gy. The results from this broad dose range were used to show that certain Raman peaks varied over this dose range 
in a way that could be fit with an induced-repair model of cell survival. There are a limited number of studies that use RS in this way, and it is therefore an avenue of research that needs further exploration.

\subsection{Thesis objectives and outline}

\subsubsection{Thesis objectives}

The work covered in this thesis centers around an exploratory study on the use of Raman micro-spectroscopy for investigating the effects of low doses of ionizing radiation on human lens epithelial (HLE) cells. The primary objectives were to determine whether biochemical differences between the mean spectrum of a given dose and the mean spectrum of the control set are statistically significant; whether individual spectra can be classified correctly between a given dose and the control set; using principal component analysis (PCA) combined with linear discriminant analysis (LDA), and leave-one-out cross-validaton (LOOCV) to estimate the classification performance; and using the spectral information these techniques provide to identify which biochemical changes contribute most to differentiating irradiated HLE cells from nonirriadiated. This latter point is important for two reasons: it provides insights into possible biological mechanisms at play that lead to cataract formation, and it provides a list of possible biomarkers that may prove useful for future investigations. The doses used were $0,0.25,0.5,2$, and 5 Gy.

Secondary objectives in this research were to see if an induced repair model of cell survival could be used to fit low-dose peak intensities, and in building a new, higher performing Raman microscope for future studies. The former objective required including data from HLE cells that received ultra-low doses of $0.01 \mathrm{~Gy}$ and $0.05 \mathrm{~Gy}$. These data were not included in the primary investigations because their 
smaller sample size limits their suitability for classification. The upgraded Raman microscope was designed specifically to address issues found with the previous apparatus during the experiment: long acquisition times and the necessity for manual mirror removal/placement when switching between brightfield imaging and Raman spectra acquisition.

\subsubsection{Thesis outline}

In chapter 1, an introduction to the issues surrounding dose limits for cataractogenesis of the human lens and the current use of Raman spectroscopy for detecting dose related changes in biological samples is given, followed by the objectives and outline of this thesis. Chapter 2 covers some basic concepts relating to ionizing radiation and radiobiology, with the bulk of the focus on the latter, including a brief summary of the current understanding of radiobiology with respect to HLE cells. In chapter 3, a classical explanation of Raman scattering and a description of Raman spectroscopy, including the technologies and techniques needed to produce a Raman spectrum, are given, followed by an overview of the multivariate statistical methods used to classify between different sets of Raman spectra. The materials and methods used to conduct the experiment are given in chapter 4, including the experimental procedure, an overview of the Carleton Raman Microscope, and a description of data preprocessing techniques used. The results of the experiment are given in chapter 5. It provides plots of the mean spectra, with $2 \sigma$ confidence intervals, for each dose compared to control; a table of the cross-validation classification accuracies found and tables of biochemicals associated with Raman shift peaks that were found to contribute to the discrimination between dose and control; and curve fits of peak intensity ratios using an induced repair model of cell survival. Chapter 6 deals with the upgraded Carleton Raman Microscope, including the reasons for the upgrade, what changes were made, 
and the performance of the new system on common test samples. Conclusions are given in chapter 7 followed by appendices that provide some derivations and a few extra figures. 


\section{Chapter 2}

\section{Ionizing Radiation and Radiobiology of HLE cells}

\subsection{Ionizing Radiation}

Ionizing radiation is any form of radiation that can lead to ionizations in atomic matter, that is, to excite an electron enough to eject it from the atom. Such an electron is no longer in any orbital of the atom from which it was ejected, and is considered a free electron.

\subsubsection{Types of ionizing radiation}

There are two types of ionizing radiation: directly ionizing radiation in the form of charged particles, and indirectly ionizing radiation in the form of electromagnetic (EM) radiation and neutron radiation. Energy levels of greater than $124 \mathrm{eV}$ are typically required for electromagnetic radiation to be considered ionizing radiation, even though the ionization energy of valence electron shells on many atoms is much 
less than this $(4-25 \mathrm{eV})^{29,30}$ and can be ionized by UV $(3.1 \mathrm{eV}-124 \mathrm{eV})$ light. $^{29,31}$ This arbitrary cutoff is primarily due to history and the non-penetrating nature of UV light, and does not apply to charged particles, for which any freely propagating charged particle capable of ionizing an atom is considered ionizing radiation.

Radiation in the form of charged particles typically comes in the form of alpha and beta radiation, which are helium nuclei and free electrons/positrons respectively. While nuclear fragments from fission, and more exotic particles (e.g. pions and other short lived composite particles) formed from cosmic rays colliding with atoms in the upper atmosphere exist, they tend to be in quantities insufficient to worry medical physicists, except in rare circumstances. Astronauts exposed to cosmic rays would be one such instance. Alpha and beta particles are emitted from unstable nuclear isotopes, with the former typically emitted at around $4 \mathrm{MeV}$, and the latter being emitted over a broad range of energies. These particles tend to only travel a short distance in any solid or liquid material, since they slow down as they lose energy, resulting in a limited penetration for a given energy level. Furthermore, their interaction cross section increases as they lose energy, increasing their linear energy transfer (LET - the amount of energy transferred from particle to material per unit distance) as they slow down.

Electromagnetic radiation, in the form of gamma rays and $\mathrm{x}$ rays, deposits energy differently. $\mathrm{X}$ rays and $\gamma$ rays have four primary modes of interaction, depending on their energy: absorption via the photoelectric effect, Rayleigh scattering, Compton scattering, and pair production. The photoelectric effect occurs when an incident photon is wholly absorbed by an electron, ejecting it from an atom. The energy of the ejected electron is then the energy of the absorbed photon minus the binding energy of the electron to the nucleus. Rayleigh scattering occurs when a photon interacts with the whole atom, that is all the orbital electrons of the atom interact constructively with the photon, and is thus referred to as coherent scattering. Since the orbital 
electrons remain bound in this case, this is the only interaction in which ionization does not occur. The photon energy remains essentially unchanged as the amount lost transferring momentum to the atom is minute (photon momentum is $h \nu / c$ ). Compton scattering, also called incoherent scattering, occurs when a photon scatters off of an electron, depositing the necessary amount of energy to satisfy conservation of momentum, and thus freeing the electron. For lower energy photons, this can only occur with valence electrons. The fourth process, pair production, requires photon energies in excess of $1.022 \mathrm{MeV}$ (mass-energy of an electron-positron pair), and occurs when a photon interacts with charged particles, typically by passing close to an atomic nucleus, and is annihilated, with an electron-positron pair being created.

The attenuation coefficient of a material with respect to photons of a given energy is the coefficient, $\mu$, required to explain the drop in beam intensity from $I_{0}$ to $I$ for a narrowly collimated beam of monoenergenic photons passing through a sheet of thickness $z$. This is called the narrow beam geometry technique, ${ }^{30}$ in which the change in intensity per unit length at a given depth in the material is given by

$$
\frac{d I}{d z}=-\mu I
$$

Thus, for a homogenous material (i.e. constant $\mu$ ), $\mu$ relates to $I_{0}$ and $I$ via

$$
I=I_{0} e^{-\mu z}
$$

The coefficient $\mu$ is often called the total attenuation coefficient since it can be written as the sum of the individual attenuation coefficients for each interaction type, i.e. $\mu=$ $\mu_{a}+\mu_{r}+\mu_{c}+\mu_{p}$, where the summed attenuation coefficients are those for absorption, Rayleigh scattering, Compton scattering, and pair production respectively. Note that, for a given material, these values change as a function of photon energy, as seen in Figure 2.1. 


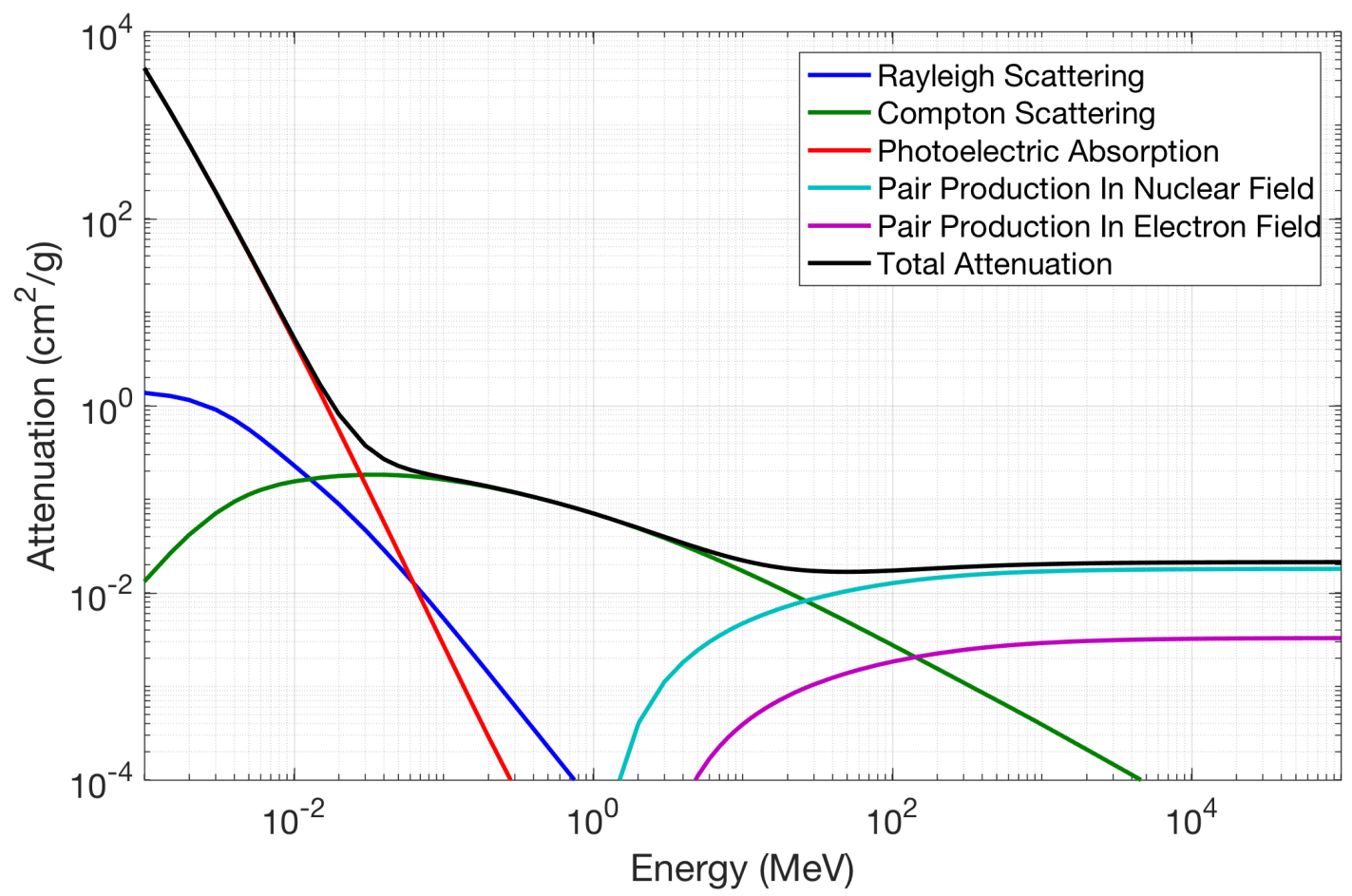

Figure 2.1: Mass attenuation coefficients for water as a function of photon energy. Data for generating plot was taken from the National Institute of Standards and Technology XCOM database of photon cross sections. ${ }^{32}$

Rather than plotting attenuation coefficients for each interaction as a function of photon energy, Figure 2.1 uses the mass attenuation coefficients instead. This relates to the individual attenuation coefficients via ${ }_{m} \mu_{i}=\mu_{i} / \rho,{ }^{30}$ where $\rho$ is the density of the material being irradiated, and $i$ is the interaction being considered. A higher mass attenuation coefficient for a given interaction type means a higher number of photons interacting via that type. Looking at Figure 2.1, we see that for $120 \mathrm{keV}$ photons in water, Compton scattering dominates. At lower energies, such as $20 \mathrm{keV}$, photoelectric absorption dominates, and pair production at high energies greater than $25 \mathrm{MeV}$.

Many researchers prefer the concept of interaction cross section when considering photon interactions with matter. The interaction cross section, ${ }_{j} \sigma_{i}$ for interaction type $i$ and target type $j$ (molecule, atom, or electron), is the cross sectional area 
each target would require to satisfy $\mu_{i}=n_{j j} \sigma_{i}$, where $n_{j}$ is the number density of the target type, that is the number of targets per unit volume. Thus, in the case of atomic and molecular cross sections, it relates to the mass attenuation coefficient by

$$
{ }_{m} \mu_{i}=\frac{{ }_{j} \sigma_{i}}{A_{j}}
$$

where $A_{j}$ is the average mass of an individual target type in the sample. For example, with an atomic cross section, ${ }_{a} \sigma_{i}$, the value of $A_{a}$ would correspond to the average atomic mass for that element in the given absorber. In the case of electronic cross sections, $e_{e} \sigma_{i}$, Equation 2.3 does not hold since most of the absorber's mass comes from the nuclei, not the electrons. In this case, we can just relate electronic cross section to atomic cross section via ${ }_{a} \sigma_{i}=Z_{e} \sigma_{i}$, where $Z$ is the atomic number.

\subsubsection{Absorbed dose}

Dose is defined as energy absorbed per unit mass. In SI units, it is given in Gray (Gy), where $1 \mathrm{~Gy}=1 \mathrm{~J} \mathrm{~kg}^{-1}$. Energy is considered "deposited" by a particle into a volume when none of it escapes the volume in the form of ionizing (primary or secondary) particles. Thus, if a particle enters a volume, and produces a tree of secondary particles, perhaps including itself if it is scattered, then the total dose deposited in that volume is the energy of all particles that escape the volume subtracted from the incident and divided by the mass in the volume. This is shown in Figure 2.2. The final form of the deposited energy is then in the form of heat, as well as in some possibly higher potential molecular formations. Any energy escaping in form of non-ionizing radiation, like light, is ignored since the energy in such cases is minute compared to the incident particle (eV vs keV and higher).

In this simplified illustration, an incident $\gamma$-ray is Compton scattered off of an 


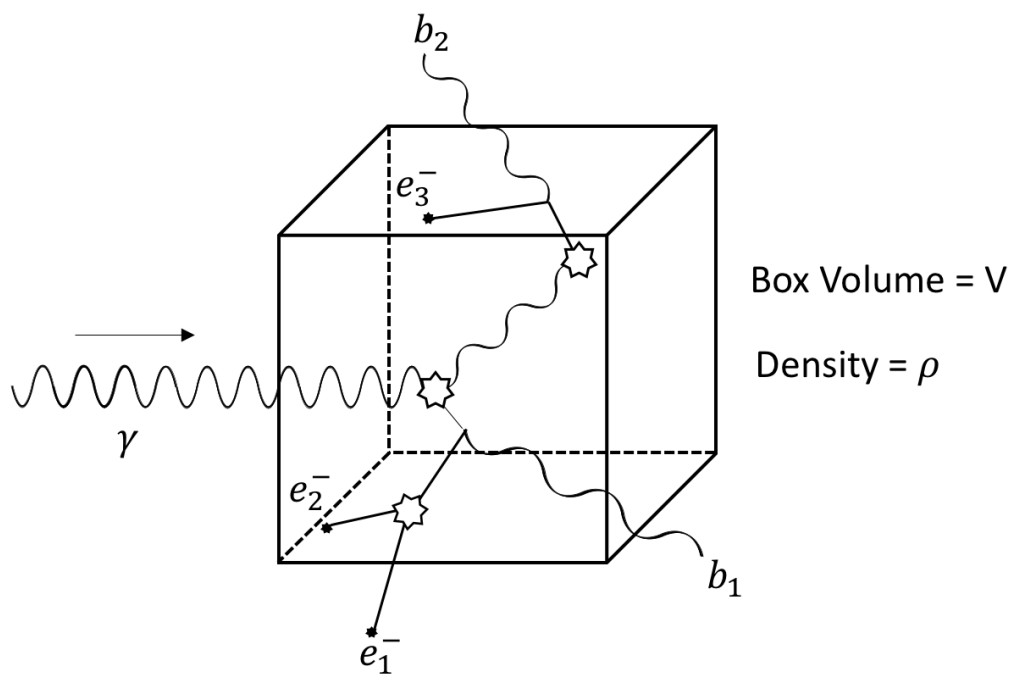

Figure 2.2: Absorbed dose in a small volume. This illustration is simplified to convey the concept of absorbed dose; free-electron paths will typically be very tortuous, with many interactions before absorption.

electron, freeing it. The path of the freed electron deflects twice and escapes the volume $\left(e^{-}\right)$, releasing some bremsstrahlung $\left(b_{1}\right)$ which also escapes the volume. It also ionizes an atom along its path. The freed electron travels a short path before being reabsorbed. The scattered photon from the initial Compton scatter travels a short distance before being absorbed and releasing a free electron which travels some distance, emitting bremsstrahlung $\left(b_{2}\right)$ along with way, before being absorbed in the volume. The absorbed dose in this volume is thus

$$
D=\frac{E_{i n c}-E_{e s c}}{m}=\frac{E_{\gamma}-\left(E_{e^{-}}+E_{b_{1}}+E_{b_{2}}\right)}{V \rho} .
$$

Note that a freed electron would typically follow a much more tortuous path than those shown here, with many more interactions before absorption, but this illustration is primarily for conceptualizing the concept of absorbed dose, and not realistic radiation transport paths based on linear energy transfer. 


\subsection{Biological effects of radiation exposure}

\subsubsection{Cell composition and DNA}

The living components of an organism are referred to as cells, and typically form themselves structurally as tissue, or suspended in liquid, as is the case with blood. Cells are the fundamental biological unit of all life forms. Multicellular life, such as plants and animals, consist of trillions of cells. Animal cells are eukaryotic cells, meaning they have membranes and all the cellular organelles are compartmentalized. Cells that are capable of dividing and thus proliferating, of producing proteins and other chemicals necessary for certain biological functions, and of differentiating themselves into other cell types, must contain a nucleus as one of their organelles.

A typical cell with a nucleus consists of an outer membrane, made of proteins and lipids, and an internal volume of organelles suspended in cytosol, the intracellular fluid of the cell consisting mainly of water. The organelles along with the cytosol, excluding the nucleus, make up what is referred to as cytoplasm. The nucleus sits at the centre of the cell and has a membrane of its own, and is typically spherical in shape. Inside the nucleus is housed Deoxyribonucleic acid (DNA), and nucleoli, a spherical substructure that forms in certain phases of the cell cycle within which ribosomal proteins are synthesized. Ribosomes are complexes of proteins and ribonucleic acid (RNA), that synthesize proteins for the cell.

The molecular composition of cells consists of water, organic (carbon containing) molecules, and inorganic molecules. Water typically makes up 70-80\% of a cell by mass, while the inorganic molecules, primarily ions of sodium $\left(\mathrm{Na}^{+}\right)$, potassium $\left(\mathrm{K}^{+}\right)$, calcium $\left(\mathrm{Ca}^{+}\right)$, chloride $\mathrm{Cl}^{-}$, bicarbonate $\left(\mathrm{HCO}_{3}^{-}\right)$, and phosphate $\left(\mathrm{PO}_{4}^{3-}\right)$, account for $<1 \% .{ }^{33}$ The organic molecules come in the form of proteins, lipids, carbohydrates, and nucleic acids (DNA and RNA), and make up the remainder of a cell's mass. Lipids 
can act as a fuel for some cells (e.g. muscle cells) and with certain types of proteins are used as structural materials for membranes and organelles, and are also produced for uses outside the cell. Other types of proteins have important internal functions of the cell, including molecular transport, metabolic functions, DNA replication and repair, and signalling within and between cells. They are composed of amino acids chained together by peptide bonds. Carbohydrates act as fuel, and for humans come in the form of glucose and glycogen, a polysaccharide of glucose molecules. In humans, only the liver and muscles store glycogen, so for most cells, glucose will be the only carbohydrate present.

DNA is the molecule that encodes the information that defines an organism genetically. It consists of two twisting, antiparallel strands connected by hydrogen bonds and forming a double helix. Each strand consists of a backbone of alternating phosphate-sugar molecules with equally spaced DNA bases connected along it. DNA bases are nitrogen containing single ring molecules (pyrimidines) and double ring molecules (purines). Each base on one strand is connected to a base on the other with hydrogen bonds, forming a base pair. There are four nucleobases, adenine (A), cytosine $(\mathrm{C})$, thymine $(\mathrm{T})$, and guanine $(\mathrm{G})$. Adenine and guanine are the purines, while cytosine and thymine are the pyrimidines. Adenine can only pair with thymine, and cytosine can only pair with guanine, meaning that there are only two types of base pairs, AT and CG. A gene is a sequence of DNA bases along a given strand, and thus all genetic information is encoded in these nucleobases.

Except for certain phases of the cell cycle, DNA is typically coiled around pill shaped proteins called histones. This packs the extremely long DNA strands into shorter structures called chromatids. In humans, two identical chromatids joined near the centre by a centromere form what is called a chromosome. A single human chromosome - there are 46 in total - thus has an X-like appearance, except for the single male Y chromosome. During genetic replication, required for a cell to split 
into two new cells under mitosis, the chromosomes break apart into their separate chromatids, within which the DNA then unravels from the histones allowing for the transcription necessary for replication.

\subsubsection{The cell cycle}

Mitotic cells are cells that undergo mitosis $(\mathrm{M})$, which is the splitting of a cell into two new cells, and is how cells proliferate. Mitosis is just one stage in the cell cycle that all mitotic cells undergo. The other three stages are Gap $1\left(\mathrm{G}_{1}\right)$, Synthesis (S), and Gap $2\left(\mathrm{G}_{2}\right)$. If a cell leaves the cell cycle, i.e. becomes temporarily or permanently non-mitotic, it enters a stage called cell cycle arrest $\left(\mathrm{G}_{0}\right)$. The full cell cycle sequence is $\mathrm{G}_{1}-\mathrm{S}-\mathrm{G}_{2}-\mathrm{M}$. In the $\mathrm{G}_{1}$ phase, all cellular contents except for chromosomes are duplicated. In the S phase, all 46 chromosomes are duplicated. Stage $\mathrm{G}_{2}$ acts as a double check of the replication done in the $\mathrm{S}$ stage, checking the duplicated chromosomes for errors and repairing as necessary.

\subsubsection{Radiation induced free radical formation in water}

Since water makes up the bulk of a cell and its nucleus, it is worth considering what happens when water interacts with ionizing radiation. When an $\mathrm{x}$ ray photon is incident on water $\left(\mathrm{H}_{2} \mathrm{O}\right)$, ionization of one of the component atoms, say through absorption, leads to dissociation of the water molecule into free radicals in the form of monatomic hydrogen $\left(\mathrm{H}^{\bullet}\right)$ and hydroxyls $\left(\mathrm{OH}^{\bullet}\right)$. An example of one such process occurs as follows:

$$
\begin{array}{r}
\mathrm{H}_{2} \mathrm{O}+\gamma \rightarrow \mathrm{H}_{2} \mathrm{O}^{+}+\mathrm{e}^{-} \\
\mathrm{H}_{2} \mathrm{O}^{+} \rightarrow \mathrm{H}^{+}+\mathrm{OH}^{\bullet}
\end{array}
$$


and

$$
\mathrm{H}^{+}+\mathrm{e}^{-} \rightarrow \mathrm{H}^{\bullet}
$$

The ions generated in this process, $\mathrm{H}^{+}$and $\mathrm{OH}^{-}$, are short lived $\left(10^{-10} \mathrm{~s}\right)$, quickly finding each other and forming a new water molecule with some energy being released. The hydroxyl $\left(\mathrm{OH}^{\bullet}\right)$ radicals, however, typically exist for a longer amount of time $\left(10^{-9} \mathrm{~s}\right)$, and therefore have more time to migrate to and damage nearby biological materials. Free radical migration distances in yeast cells have been estimated to be between tens and hundreds of $\AA .{ }^{34}$ DNA radicals, formed similarly or by interacting with $\mathrm{OH}^{\bullet}$ radicals have an even longer lifetime of $10^{-5} \mathrm{s.}^{1}$

The $\gamma$-ray in Equation 2.5 could be replaced by a free electron. Also, the ejected electron could go on to dissociate more $\mathrm{H}_{2} \mathrm{O}$ molecules if of sufficient energy, meaning a chain reaction can follow until all of the incident energy is dissipated. While these electrons ejected can also damage materials directly, for every free electron with keVs of energy or greater, many free radicals will be formed along its path. Thus, there will be an abundance of free radicals per free electron, and correspondingly the majority of physical damage to surrounding biological material will be due to these free radicals.

\subsubsection{DNA damage}

While ionizing radiation and the free radicals it produces can damage many cellular constituents, such as proteins and lipids in the membrane, or part of organelles, such damage is generally considered to be less significant than damage to DNA due to both the fact that such materials are abundant and their role less critical on a single molecular level. ${ }^{35}$ As a store of information necessary for proper cell functioning, damage to DNA can be catastrophic to a cell. Ionizing radiation can damage DNA directly by ionizing portions of a DNA molecule, for example an electron cutting a 
path through a double helix, or via free radicals that chemically change a portion of a strand, altering or breaking its structure. The free radicals can bind to DNA molecules leading to lesions and single or double-strand breaks (SSBs and DSBs) in the double helix. A double strand break is a break in the DNA backbone on each strand that is only a few base pairs away from each other, allowing for total separation of the DNA molecule at that region. It can occur instantly as when a charged particle of sufficient energy plows through both strands, or by two SSBs occurring near enough to be considered a DSB.

DNA damage in the form of DSBs is largely considered to be the primary form of cell damage that leads to cell death from radiation exposure. ${ }^{1}$ One study by Richardson et $a l^{36}$ suggested that low-LET radiation-induced effects in mitochondria lead to the emission of reactive oxygen species from the mitochondrial electron transport chain that go on damage nuclear DNA, and that this is the primary mechanism by which cells die from low-dose radiation exposure. The point remains that DNA damage is still required for lethality, but this suggests that mitochondria are the key target inducing cell death using low-dose x rays.

Damage to nuclear DNA can come in two forms, stable and unstable. Stable damage to DNA causes mutations which can lead to cells becoming cancerous, or, if the mutation occurs in germ cells, cause debilitating illnesses in offspring. ${ }^{37,38}$ Unstable damage is any damage to DNA that leads to either cell death through apoptosis, necrosis, autophagy, or cellular senescence. ${ }^{39}$ DNA damage is a common occurrence, however, with an estimated 10 DSBs occurring per day per mitotic cell on average. ${ }^{40}$ To deal with this, mammals have advanced DNA repair mechanisms that allow for reconnection of broken strands. 


\subsubsection{Cellular response}

DNA damage can be detected by sensor proteins that, upon detecting damage to the genome, signal three main effector pathways: apoptosis, which is programmed cell death; DNA repair pathways, and checkpoints leading to temporary or permanent blocks in the cell cycle (cellular senescence). A well-known initiator of DSB repair pathways is the histone H2AX. The sensing process consists of H2AX being quickly phosphorylated into $\gamma \mathrm{H} 2 \mathrm{AX}$ at the site of a DNA DSB, acting as a marker. It attracts DNA repair proteins which then go about repairing the damaged strands. ${ }^{35,41}$

For simple damage to DNA bases and SSBs, base excision repair (BER), SSB repair (SSBR), are used. ${ }^{1}$ Nucleotide excision repair (NER) for damage nucleotides and mismatch repair (MMR) for mismatched bases are less relevant repair processes with respect to radiation damage. BER and SSBR are relatively simple processes in which the non-damaged strand can be used as a template. ${ }^{1}$ For this reason, DNA base damage and SSBs are usually not an issue. At high dose rates, SSBs may accumulate and become DSBs, however.

Double strand breaks tend to be more difficult to repair. They are usually repaired by either homologous resection (HR), non-homologous end joining (NHEJ), or single strand annealing (SSA). ${ }^{35}$ NHEJ is used more often in mammals, partially because it can be done throughout the cell cycle, but it less accurate than HR, and can lead to genetic and chromosomal aberrations. HR is only available in the S and $\mathrm{G}_{2}$ phases of the cell cycle. If the mistakes in the DNA repair are large enough, as is the case in chromosomal aberrations, they lead to eventual cell death at mitosis, while smaller genetic defects exist as mutations which propagate through the life cycle of the cell. Single strand annealing has not been researched as extensively as the other repair mechanisms.

Another way in which cells respond to radiation damage is by releasing signalling 
factors that induce what is called the bystander effect, which is defined as the induction of biologic effects in cells that are not directly traversed by a charged particle, but are in proximity to cells that are. ${ }^{1}$ This is seen in experiments done on cell cultures in which a portion of cells will be irradiated but an increase in cell death is seen not just in the irradiated fraction of cells, but in the non-irradiated fraction as well. The belief that signalling factors are secreted by the irradiated cells and that induce apoptosis in the non-irradiated is due to studies in which solution from a sample of irradiated cells was extracted after irradiation and added to a non-irradiated sample, inducing the same increase in cell mortality. ${ }^{42}$ Irradiating samples with no cells, and transferring the solution was used as a control, and showed no such effect.

\subsubsection{Survival curves and the linear quadratic model of cell survival}

Cell survival curves are plots of cell count vs dose. They show the relationship between cell survival (or death) as a function of dose received for a given type of IR. They are created by plotting cell count vs dose on a plot, and fitting the data points with a function that describes the relation theoretically. The linear quadratic model of cell survival is usually what is used to fit such data. This model is defined by the equation

$$
S=e^{-\alpha D-\beta D^{2}}
$$

where $S$ is the surviving fraction. This equation can be derived by applying target theory of radiation action to DNA DSBs. ${ }^{43}$ In this model, there are two types of events assumed to lead to cell death: single lethal events, proportional to dose, $D$, with the constant of proportionality $\alpha$, and accumulated sublethal events, proportional to $D^{2}$, with the constant of proportionality $\beta$. These two modes of cell death are assumed to be independent of one another. ${ }^{1}$ 


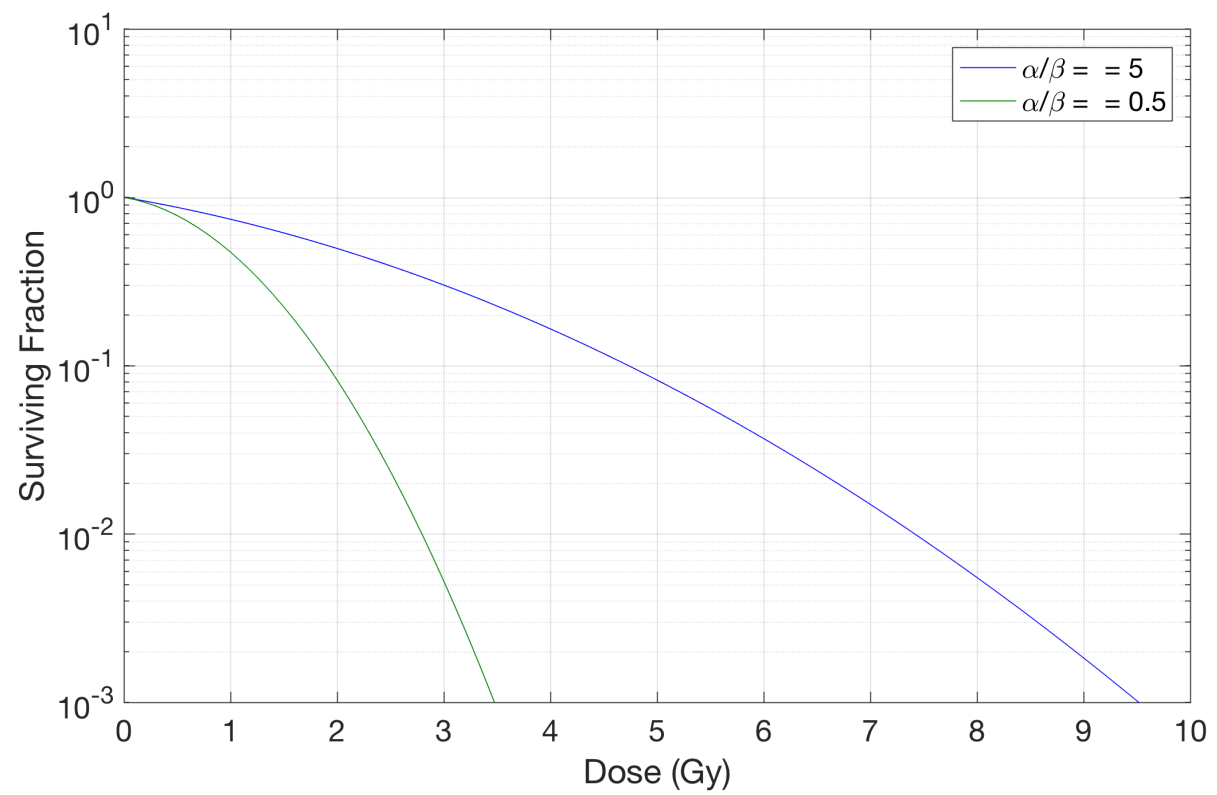

Figure 2.3: Linear quadratic model of cell survival for different $\alpha / \beta$ values. Surviving Fraction axis is log scale.

An example of a cell survival curve with different $\alpha / \beta$ values is given in Figure 2.3. These curves would typically be fit to clonogenic assay data. Such an assay requires multiple cell cultures to be prepared, irradiated at different doses, and then the number of surviving cells as a fraction of the original cell count plotted as a function of dose. While it is clear that such curves allow one to model cell death as a function of dose, they are not used for in vivo dosimetric analysis.

\subsubsection{Low-dose hypersensitivity and induced radioresistance}

Low-dose hypersensitivity, also referred to as hyper-radiosensitivity (HDR), is a phenomenon in which cells show increased radiosensitivity at doses below 0.5 Gy, followed with a relative increase in radioresistance for doses between 0.5 and 1 Gy. ${ }^{44}$

While the mechanisms underlying low-dose hypersensitivity and increased radioresistance are not fully understood, Joiner and Johns (1988) suggested a model 
based on induced repair to explain renal damage in irradiated mouse kidneys. ${ }^{45}$ The equation for the survival curve based on this induced repair model is given by

$$
S=\exp \left\{-\left[\alpha_{H}\left(1+\left[\frac{\alpha_{L}}{\alpha_{H}}-1\right] \exp \left(-\frac{D}{D_{C}}\right)\right)\right] D-\beta D^{2}\right\},
$$

where $\mathrm{S}$ is the surviving fraction of cells as before, but there are now two $\alpha$ terms, $\alpha_{L}$ and $\alpha_{H}$. The parameter $\alpha_{H}$ corresponds to the $\alpha$ parameter in the standard LQ model of cell survival that fits well to high doses, while $\alpha_{L}$ corresponds to a different, higher rate of unrepairable damage leading to cell death. What this model actually assumes, however, is that it is the repairability of the same type of damage that changes. It assumes a dose threshold, given by the constant $D_{C}$, must be reached before certain repair processes kick in. ${ }^{45}$

To understand how Equation 2.8 will differ from the LQ model given by Equation 2.7, we need look at the inner part of the equation contained within the brackets, which replaces the previous $\alpha$ parameter. If we multiply $\alpha_{H}$ through, this becomes

$$
\alpha_{H}+\left[\alpha_{L}-\alpha_{H}\right] \exp \left(\frac{D}{D_{C}}\right)
$$

We see then that for $D<<D_{C}$, this expression becomes approximately equal to $\alpha_{L}$, and so Equation 2.8 becomes equivalent to Equation 2.7 with $\alpha=\alpha_{L}$ at low doses. At higher doses, with $D>>D_{C}$, the expression becomes approximately equal to $\alpha_{H}$, and so the $\alpha_{L} / \alpha_{H}$ ratio, determines the amount of increased sensitivity. It is clear that Equation 2.8 reduces to Equation 2.7 for all doses when $\alpha_{L}=\alpha_{H}$. 


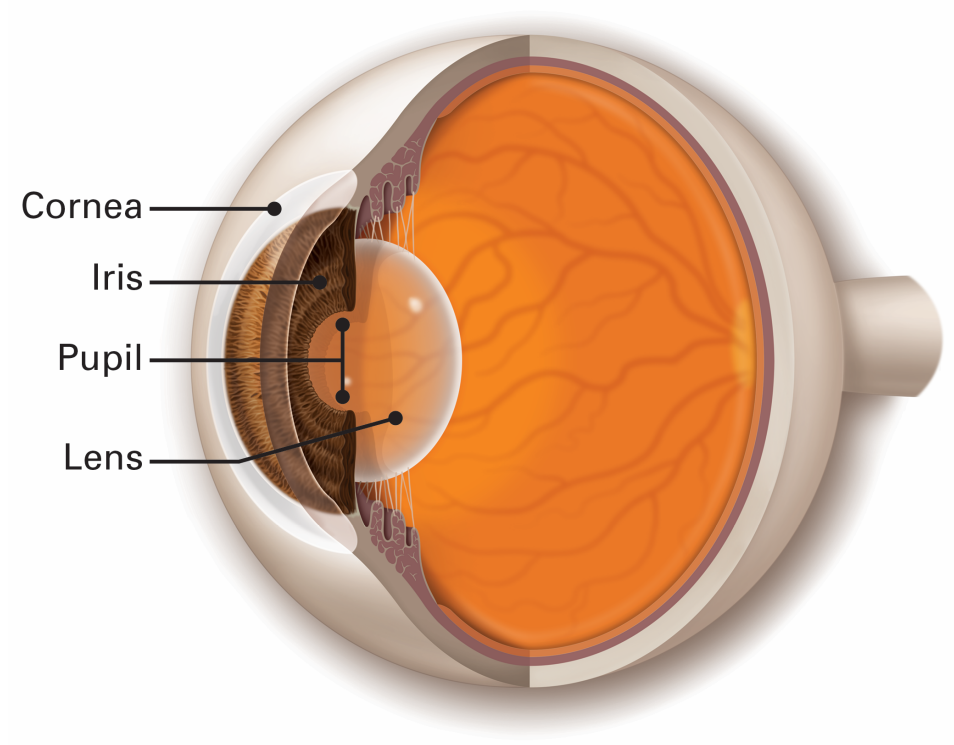

Figure 2.4: Diagram of human eye, showing lens position relative to cornea and retina (with permission from American Academy of Ophthalmology).

\subsection{Human lens epithelial cells}

The lens of the eye consists of two types of cells, lens fiber cells, and lens epithelial cells. It sits behind the cornea and is responsible for focusing light onto the retina, which absorbs photons and transmits the image information to the back of the brain via the optic nerve. A diagram of a human lens sitting in an eye is given in Figure 2.4. The lens is shaped as a biconvex lens, with slightly more curvature towards the posterior. It is flexible in youth, using muscles with string-like zonules connected along its edges to pull on it, stretching it flat and thin, stretching it out, or to let it relax, allowing it to bulge. This ability to change thickness and shape is how the lens changes its focal length, allowing it to focus on objects at various distances. Over time, the lens fibers become less stretchable, and thus the focal range of individuals decreases with age, with many older people often requiring reading glasses, a condition known as presbyopia. Another age related change is the formation of cataracts, in which lens fibers develop opacities. Cataract formation can also be induced by radiation. 


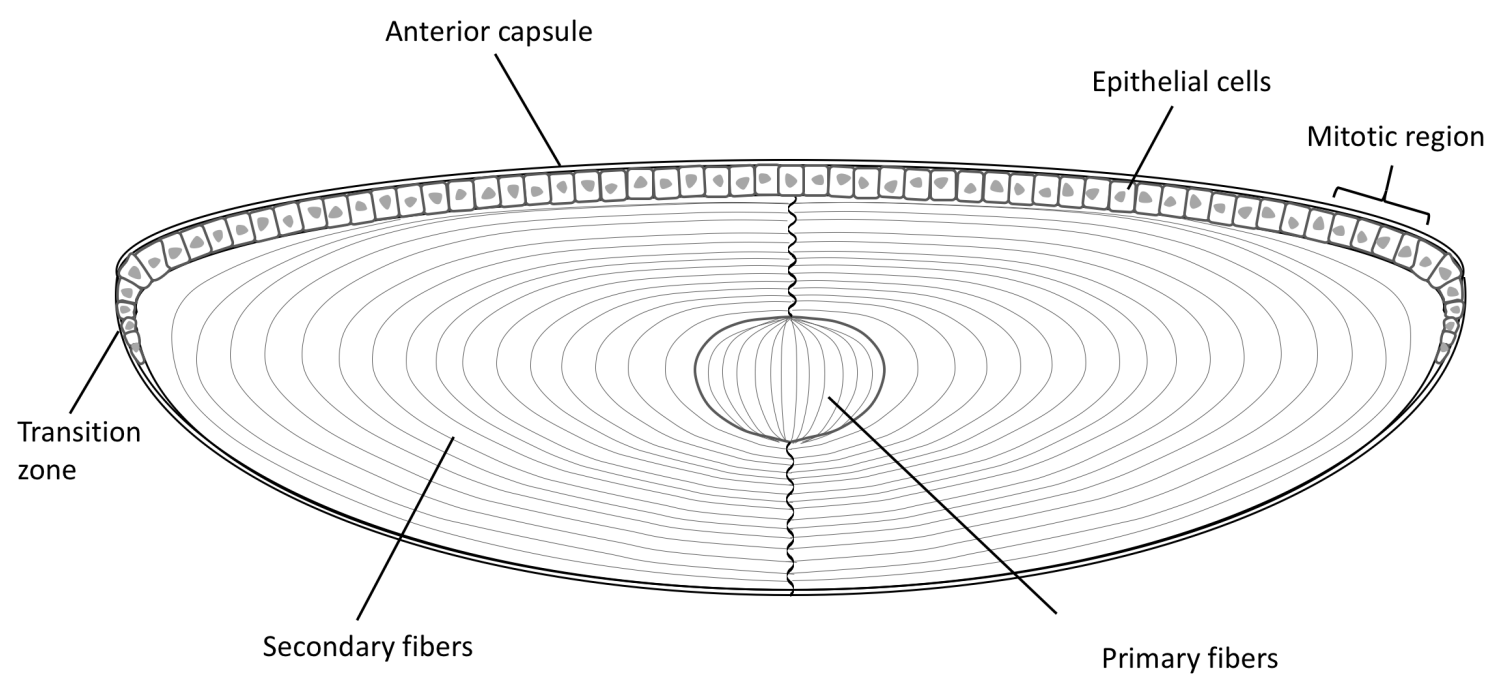

Figure 2.5: Diagram of human lens, showing fiber structure and location of epithelium. Epithelial cells in the transition zone differentiate into lens fiber cells.

The lens fiber cells are not metabolically active, and contain no nuclei. They make up the bulk of the lens, and act as its refractive material. The lens epithelial cells sit as a single layer on the front and sides of the lens, as shown in Figure 2.5. These cells are metabolically active, maintaining homeostasis of the lens as whole. They are also responsible for the production of lens fiber cells, which they differentiate into. As part of maintaining homeostasis, they produce antioxidants such as glutathione. New LE cells are formed at the centre of the front layers, and drift outward over time, differentiating into LF cells at the posterior.

Unlike most tissues, the lens has no mechanism for cell removal. Damaged HLE cells will therefore remain and may differentiate into abnormal and possibly opaque LF cells. When this occurs enough to become visible and impair vision, a cataract is said to have formed.

The human lens is one of the most radiosensitive ocular tissues, ${ }^{5,46}$ with cataracts readily forming in the eyes of people who have received high exposures in radiological incidents. ${ }^{1}$ While the mechanisms leading to cataract formation after irradition are not yet fully understood, studies have shown that it may be related to activation of 
oxidative stress pathways, leading to DNA damage to HLE cells, causing an increase in cell proliferation. ${ }^{5}$ Studies have shown these increases in cell proliferation and differentiation due to radiation exposure may be in part due to slow DNA repair. ${ }^{47}$ Since the bulk of the epithelium sits at the front of the lens, it also receives a higher dose than the rest of the lens when exposed to EM ionizing radiation. At lower doses, cataractogenesis may take years to develop. How long term accumulation of dose increases the chance of cataract formation is poorly understood. A better understanding of the basic biochemical changes that occur in cells that receive low doses of radiation might illuminate biological pathways that lead to formation of opacities in the lens. 


\section{Chapter 3}

\section{Raman spectroscopy and}

\section{multivariate statistical analysis}

\subsection{Raman spectroscopy}

\subsubsection{Raman scattering}

Raman scattering is the inelastic scattering of light, as opposed to the far more common elastic scattering of light, known as Rayleigh scattering. Being inelastic, the scattered photon loses or gains some energy, which is transferred to or from molecular vibrations of the scattering molecule. In the case where the Raman scattered photon loses energy, it is called Stokes scattering, while the case in which it gains energy is called anti-Stokes scattering. Since the vibrational modes of the molecule are discrete states and are unique to a given molecule, recording the spectrum of the Raman scattered light from a molecule provides a fingerprint of it, from which it can be identified. The changes in vibrational energy of the scattering molecule and of the scattered photon are shown in Figure 3.1. 


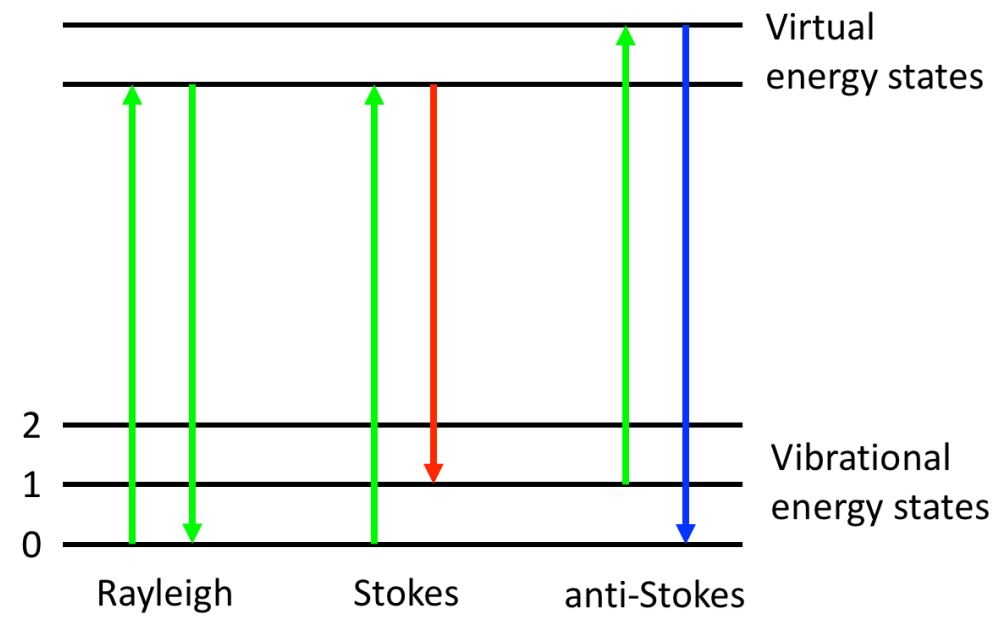

Figure 3.1: Changes in energy level of scattering molecule and scattered photon.

While a quantum mechanical description of Raman scattering is necessary to describe all aspects of it, a classical description will suffice for a simple (non-rotating, non-translating) vibrational case. Note that angular frequency, $\omega=2 \pi \nu$, will be used instead of frequency $\nu$ to simplify equations since the trigonometric terms all require factors of $2 \pi$.

In the classical description of Raman scattering, the oscillating electric field of an EM wave induces an electric dipole in a molecule. We consider the electric dipole moment vector $\mathbf{p}=\left(p_{x}, p_{y}, p_{z}\right)^{T}$, and electric field vector corresponding to the incident light, $\mathbf{E}=\left(E_{x}, E_{y}, E_{z}\right)^{T}$, which we will assume to be a monochromatic plane wave of frequency $\omega_{I}$, and thus can be written as

$$
\mathbf{E}=\mathbf{E}_{0} \cos \omega_{I} t
$$

The oscillating E-field induces a time-dependent dipole moment in a molecule, which 
can be written as a sum of dipole moment vectors of increasing order, ${ }^{48}$

$$
\begin{aligned}
\mathbf{p} & =\mathbf{p}^{(1)}+\mathbf{p}^{(2)}+\mathbf{p}^{(3)}+\ldots \\
& =\boldsymbol{\alpha} \mathbf{E}+\frac{1}{2} \boldsymbol{\beta} \mathbf{E} \mathbf{E}+\frac{1}{6} \gamma \mathbf{E E E}+\ldots,
\end{aligned}
$$

where $\boldsymbol{\alpha}$ is a 2 nd-order tensor (i.e. a matrix), called the polarizability tensor, $\boldsymbol{\beta}$ is 3rd-order tensor, called the hyperpolarizability tensor and EE is a second order tensor, and so on. Note that EE and EEE are simply tensor products of $\mathbf{E}$ with itself. The first term, $\mathbf{p}^{(1)}$, is thus a linear function of $\mathbf{E}$, while terms $\mathbf{p}^{(n)}$ with $n>1$ are non-linear functions of $\mathbf{E}$. At typical laser intensities (i.e. not those produced by femto-second pulsed lasers), $\mathbf{p}^{(1)} \gg \mathbf{p}^{(2)} \gg \mathbf{p}^{(3)} \gg \ldots$, and so only the first dipole need be considered, that is to say

$$
\mathbf{p} \approx \mathbf{p}^{(1)}=\alpha \mathbf{E}
$$

It is this first order induced electric dipole that is used to describe incoherent Raman scattering. If we allow our model of the scattering molecule to vibrate, but not rotate or translate, meaning we only allow the nuclei to vibrate around a fixed equilibrium position, then we can express the polarizability by expanding the components of the polarizability tensor as follows:

$$
\alpha_{i, j}=\left(\alpha_{i, j}\right)_{0}+\sum_{k}\left(\frac{\partial \alpha_{i, j}}{\partial Q_{k}}\right)_{0} Q_{k}+\frac{1}{2} \sum_{k, l}\left(\frac{\partial \alpha_{i, j}^{2}}{\partial Q_{k} \partial Q_{l}}\right)_{0} Q_{k} Q_{l}+\ldots
$$

where $Q_{k}, Q_{l}, \ldots$ are normal coordinates, linear combinations of mass-weighted displacement coordinates that are derived in Appendix A.1, ${ }^{49}$ relative to the atom's equilibrium position, and corresponding to normal vibrational modes with frequencies $\omega_{k}, \omega_{l}, \ldots$, at some point in time. The sums are over all normal coordinates, and the value of the derivative is taken at $Q_{k}=0$, the equilibrium position. If we con- 
sider the case of a single normal mode (i.e. no summation over all modes), and ignore powers greater than one, ie. only considering the first two terms, then Equation 3.4 reduces to

$$
\left(\alpha_{i, j}\right)_{k}=\left(\alpha_{i, j}\right)_{0}+\left(\frac{\partial \alpha_{i, j}}{\partial Q_{k}}\right)_{0} Q_{k} .
$$

This is called the electric harmonic approximation, ${ }^{48}$ and allows us to write each element in $\boldsymbol{\alpha}_{k}$ as the sum of an equilibrium and vibrational term, thus splitting $\boldsymbol{\alpha}_{k}$ into a sum of two tensors,

$$
\boldsymbol{\alpha}_{k}=\boldsymbol{\alpha}_{0}+\left(\frac{\partial \boldsymbol{\alpha}}{\partial Q_{k}}\right)_{0} Q_{k}=\boldsymbol{\alpha}_{0}+\boldsymbol{\alpha}_{k}^{\prime} Q_{k}
$$

where $\boldsymbol{\alpha}_{k}^{\prime}$ is called the derived polarizability tensor. ${ }^{48}$ If we assume simple harmonic motion for the atom, then the time dependence of the normal coordinate can be written as

$$
Q_{k}(t)=Q_{k_{0}} \cos \left(\omega_{k} t+\delta_{k}\right)
$$

where $\delta_{k}$ is a phase offset, $Q_{k_{0}}$ is the amplitude of the normal coordinate, and $\omega_{k}$ is the angular frequency of the $k^{t h}$ normal mode. With this, Equation 3.6 becomes

$$
\boldsymbol{\alpha}_{k}=\boldsymbol{\alpha}_{0}+\boldsymbol{\alpha}_{k}^{\prime} Q_{k_{0}} \cos \left(\omega_{k} t+\delta_{k}\right)
$$

Plugging this into Equation 3.3, and with the time-dependent form of $\mathbf{E}$ (Equation $3.1)$, gives

$$
\mathbf{p}^{(1)}=\boldsymbol{\alpha}_{0} \mathbf{E}_{0} \cos \omega_{I} t+\boldsymbol{\alpha}_{k}^{\prime} \cos \left(\omega_{k} t+\delta_{k}\right) \mathbf{E}_{0} \cos \omega_{I} t
$$

The last term can be simplified using the trigonometric identity

$$
\cos A \cos B=\frac{1}{2}[\cos (A+B)+\cos (A-B)]
$$


from which Equation 3.9 becomes

$$
\mathbf{p}^{(1)}=\boldsymbol{\alpha}_{0} \mathbf{E}_{0} \cos \omega_{I} t+\frac{1}{2} \boldsymbol{\alpha}_{k}^{\prime} \mathbf{E}_{0}\left[\cos \left(\left(\omega_{k}+\omega_{I}\right) t+\delta_{k}\right)+\cos \left(\left(\omega_{k}-\omega_{I}\right) t+\delta_{k}\right)\right],
$$

which can be written as

$$
\begin{aligned}
\mathbf{p}^{(1)} & =\mathbf{p}_{0}^{(1)} \cos \omega_{I} t+\mathbf{p}_{k_{0}}^{(1)} \cos \left(\left(\omega_{k}+\omega_{I}\right) t\right)+\mathbf{p}_{k_{0}}^{(1)} \cos \left(\left(\omega_{k}-\omega_{I}\right) t\right) \\
& =\mathbf{p}^{(1)}\left(\omega_{I}\right)+\mathbf{p}^{(1)}\left(\omega_{k}+\omega_{I}\right)+\mathbf{p}^{(1)}\left(\omega_{k}-\omega_{I}\right)
\end{aligned}
$$

Thus, the linearly induced electric dipole can be written as a sum of three terms with

different frequency dependencies. The first term, $\mathbf{p}_{0}^{(1)}\left(\omega_{I}\right)$, corresponds to Rayleigh scattered light in which there is no change in frequency of the scattering photons, while the other two terms correspond to Raman scattered light. Specifically, the term $\mathbf{p}^{(1)}\left(\omega_{k}-\omega_{I}\right)$ corresponds to Stokes scattered light, in which the scattered light has a lower frequency from the incident light of $\omega_{k}-\omega_{I}$, and $\mathbf{p}^{(1)}\left(\omega_{k}+\omega_{I}\right)$ corresponds to anti-Stokes scattered light, in which the scattered light has an increased frequency of $\omega_{k}+\omega_{I}$.

For spontaneous Raman scattering at room temperature, the vast majority of scatters will be Stokes, rather than anti-Stokes. This is due to the fact that very few molecules are in excited vibrational states under normal conditions. Adding to this that the probability of any Raman scatter is typically 1 in $10^{6}-10^{8},{ }^{50}$ depending on the material, means the chance of a spontaneous anti-Stokes scatter is very small. For this reason, Raman spectroscopy is typically done for stokes scattered light.

\subsubsection{Molecular vibrations}

The number of (normal) vibrational modes a molecule has depends on the number of atoms that make up the molecule and its molecular structure. If there are $N$ 
$(N>1)$ atoms in a molecule, and we assume none are in excited state, then the total number of degrees of freedom (DoF) the molecule has is $3 N$. This comes from the fact that each atom can move in 3 orthogonal directions in a 3-dimensional space, and so each atom adds 3 DoF to the system. Vibrational DoF are independent ways the atoms in a molecule can move relative to each other, with each DoF corresponding to a single vibrational mode. It therefore does not include rotation and translation of the molecule as a whole, as such motion does not change the relative distances between atoms in the molecule and thus do not count as vibrations. The number of vibrational DoF is thus found by taking the total number of DoF and subtracting those not corresponding to normal vibrational modes, namely, those corresponding to translation and rotation.

From this, we find the number of vibrational DoF is $3 N-6$ for non-linear molecules, and $3 N-5$ for linear ones. ${ }^{50,51} \mathrm{~A}$ linear molecule is one in which all the atoms are arranged in a sequential line, essentially a straight chain of atoms. It is minus 6 DoF for non-linear molecules because of the 3 independent axes a molecule can translate along in 3-dimensional space and the 3 axes it can rotate around. For linear molecules, rotation about the central axis does not change any atomic coordinates in the system and therefore is indistinguishable from a non-rotating state. ${ }^{50}$ For this reason, linear molecules only have 2 rotational DoF for a total of 5 non-vibrational DoF.

Examples of the vibrational modes of $\mathrm{CO}_{2}$ molecule are given in Figure 3.2. It has $3(3)-5=4$ normal vibrational modes.

For larger molecules, a structure made of repeating bond types will vibrate as a group. An example of this are the vibrational modes of benzene, where the group vibrations of the $\mathrm{C}_{6} \mathrm{H}_{6}$ molecule are considered as the source of a Raman scatter, and not just individual $\mathrm{C}-\mathrm{H}$ and $\mathrm{C}-\mathrm{C}$ bonds that make up the structure. This is illustrated 


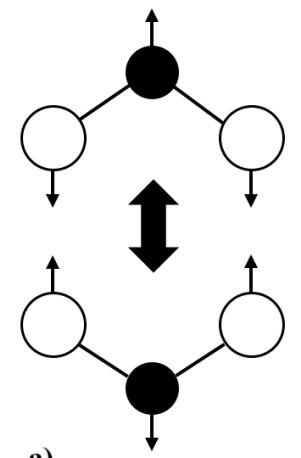

a)

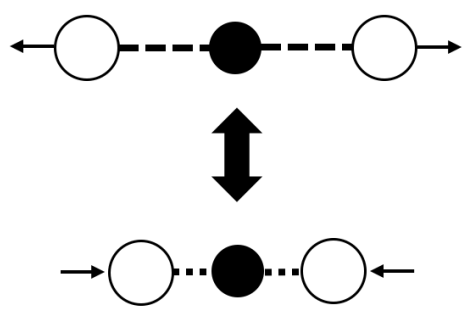

b)

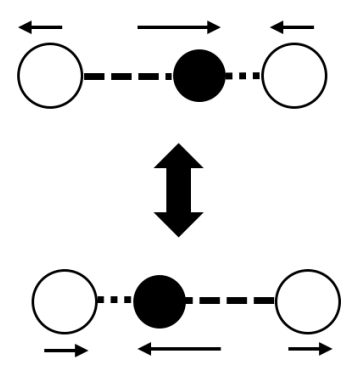

c)

Figure 3.2: Carbon dioxide $\mathrm{CO}_{2}$ vibrational modes: a) bending (deformation), which can occur along two orthogonal axes and so counts as two normal modes, b) symmetric stretch, and c) asymmetric stretch.

in Figure 3.3. These group vibrations are accounted for in normal vibrational modes, which are linearly independent modes of oscillation that can be dependent on any number of atoms in the system (see Appendix A.1). To induce a Raman scatter requires more than a scattering molecule having a set of vibrational states. The nature of vibration must allow for a change in the polarizability of the vibrating structure, and this depends on the derived polarizability tensor $\boldsymbol{\alpha}_{k}^{\prime}$ for a given normal mode. Recall from Equation 3.6, that

$$
\boldsymbol{\alpha}_{k}^{\prime}=\left(\frac{\partial \boldsymbol{\alpha}}{\partial Q_{k}}\right)_{0}
$$

and so the derived polarizability represents the amount of change induced in the polarizability of the molecule by the $k^{\text {th }}$ vibrational mode. Every normal mode will thus have its own $\boldsymbol{\alpha}_{k}^{\prime}$, and if it is non-zero, the corresponding vibration is said to be Raman active. A quantum mechanical treatment of molecular vibrations and Raman scattering is required for more rigorous selection rules of Raman-activity, but this is beyond the scope of this thesis. 

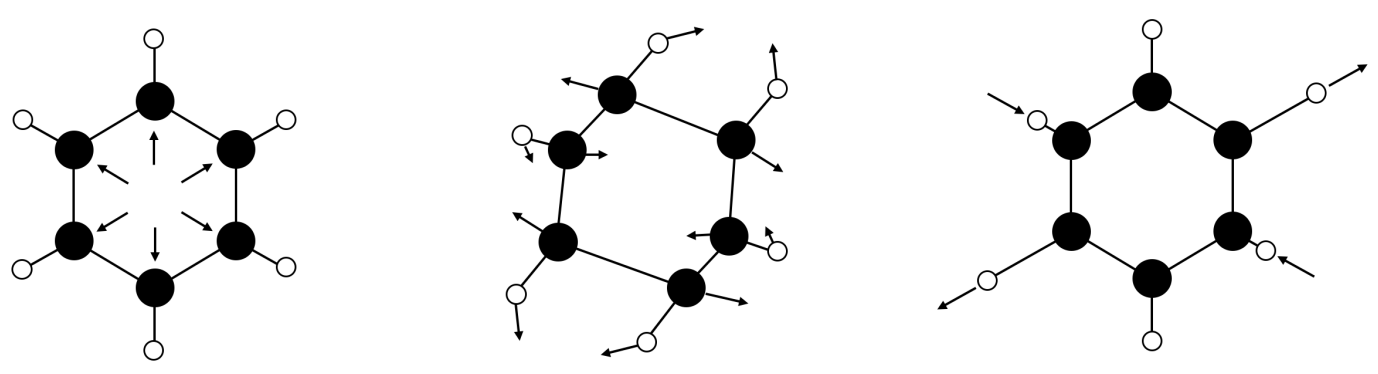

a)

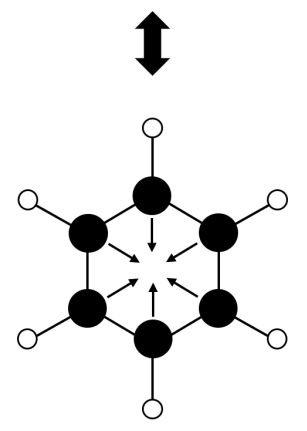

b)

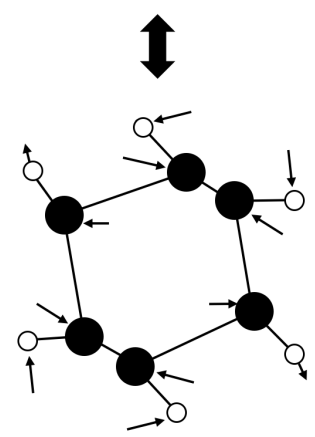

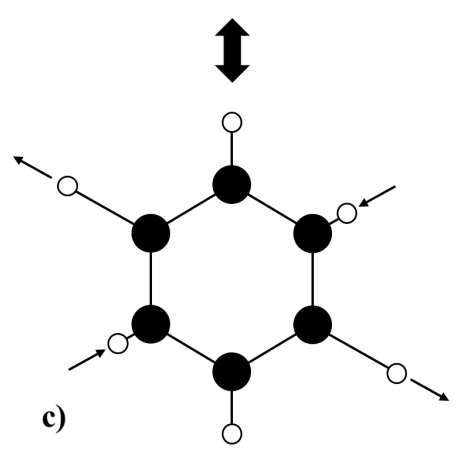

Figure 3.3: Benzene $\left(\mathrm{C}_{6} \mathrm{H}_{6}\right)$ group vibrational modes: a) symmetric breathing mode, b) a quadrant stretch, c) $\mathrm{C}-\mathrm{H}$ vibrations.

\subsubsection{Raman shift and Raman spectra}

In order to generate Raman spectra in a sample, one must have a source of monochromatic light, usually a laser, and a spectrometer. The spectrometer measures the relative intensities of the Raman scattered light over range of wavelengths, forming an initial spectrum. Because the wavelength of the laser, $\lambda_{i}$, is known, a Raman shift, which is a difference in wavenumbers between incident and scattered light, can be found:

$$
\Delta w=\frac{1}{\lambda_{i}}-\frac{1}{\lambda_{f}}=\frac{E_{i}-E_{f}}{h c}
$$

where $h$ and $c$ are Planck's constant and the speed of light, $\lambda_{f}$ is the wavelength of the scattered light, and $E_{i}$ and $E_{f}$ are the energies of the incident and scattered light respectively. Since this is a difference in wavenumbers, the final units for Raman shift are given in $\mathrm{cm}^{-1}$, as is custom in most spectroscopic techniques involving wavenumbers. The relative intensities measured over a range of Raman shift wavenumbers 
is what constitutes a Raman spectrum. Peaks in this spectrum then correspond to $E_{w} / h c$, where $E_{w}$ is the energy of a specific vibrational state in the sample.

A monochromatic light source is necessary because having incident photons of various energies produce Raman scattered light of various energies from the same vibrational state, since, for Stokes scattered light, the energy of the scattered photon is given by

$$
E^{\prime}=E_{i}-E_{w}
$$

where $E_{i}$ is the energy of the incident photon and $E_{w}$ the energy of the specific vibrational state that the molecule is set into by the scatter. Thus, for a large number of photons, varying $E_{i}$ would lead to varying $E^{\prime}$. Since one would not know what the initial energy was of each incident photon, one would therefore be unable to uniquely determine which vibrational state is responsible for the scatter. The effect would be a blurring of each Raman peak over a portion of the spectrum, the degree of which would depend on how wide the incident spectrum is.

Relatively pure materials tend to have simple Raman spectra due to every molecule in the sample having the same set of vibrational states that can be induced by the incident laser light. This is demonstrated in Figure 3.4.

Silicon, for example, has a prominent peak at $520 \mathrm{~cm}^{-1}$ which dwarfs all other nearby peaks. Polystyrene, a more complex molecule compared to silicon's single element in a crystal lattice, provides a somewhat more complex spectrum, but is still primarily dominated by a couple of prominent Raman peaks. Biological samples on the other hand, such as blood and HLE cells, produce much more complex spectra. This is due to the various constituent molecules having many vibrational modes. Biological samples tend to have large complex molecules such as proteins, lipids, and nucleic acids, and these can vibrate in many more ways than simpler materials due to their having many more atoms bonded together in various shapes. Adding to this, 


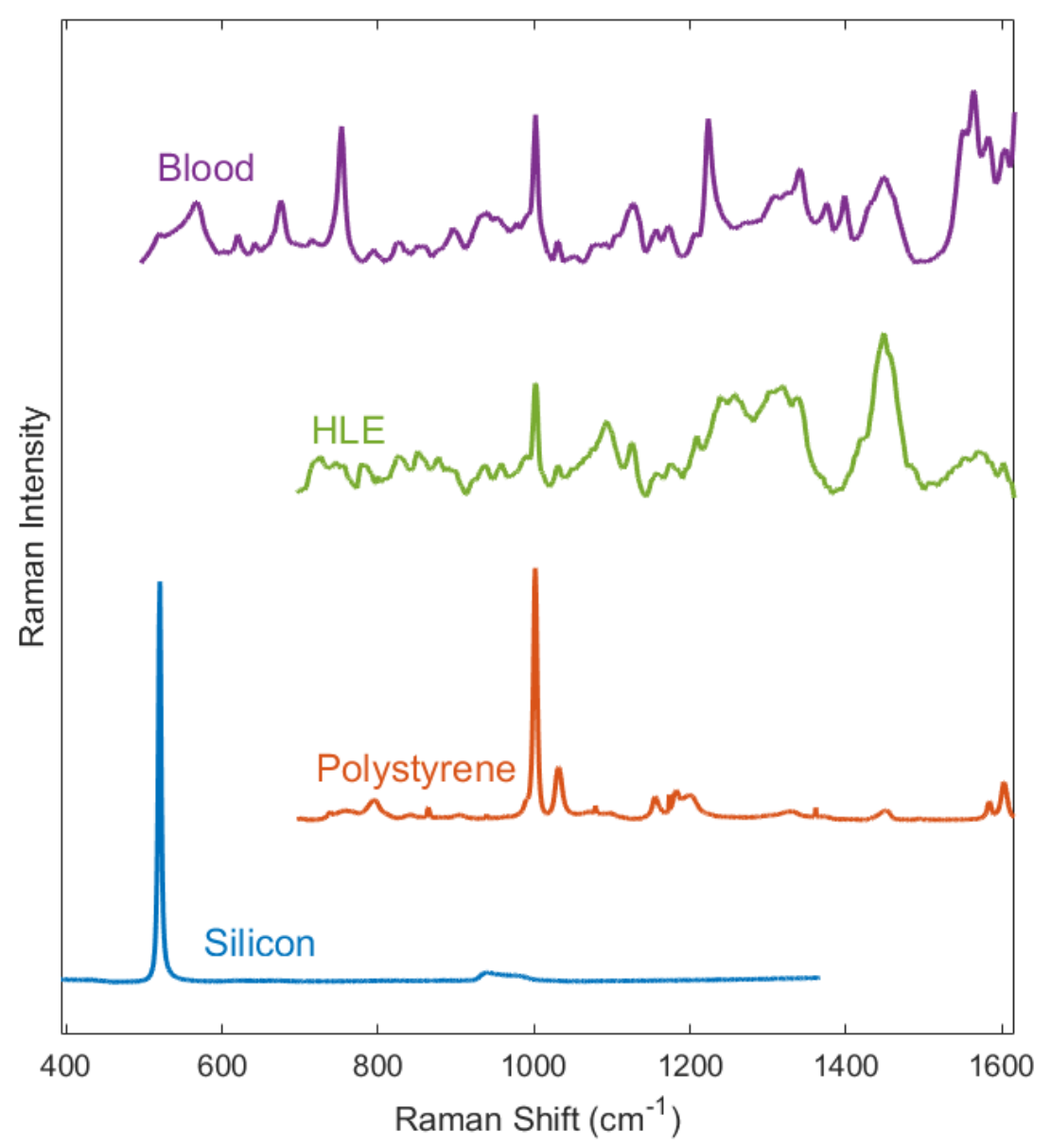

Figure 3.4: Examples of Raman spectra from various materials, demonstrating how molecular variety and complexity increases spectral complexity. Intensities are not to scale - biological sample spectra are orders of magnitude smaller for a given acquisition time.

many of these molecules have vibrational states that are close in energy or the same, which leads to spectral overlap of various peaks. This can create an effect of broad lineshapes that look almost like fluorescence. The multiple humps on such line shapes gives away their true nature as overlapping Raman peaks. 


\subsubsection{Spectrometer}

The spectrometer used in this study is of the Czerny-Turner variety, which works as follows: light enters through a slit and is reflected off of a parabolic mirror, then off of an reflective diffraction grating, which splits the Raman scattered light into its spectral components, and then off of another mirror and onto a CCD. This is shown in Figure 3.5.

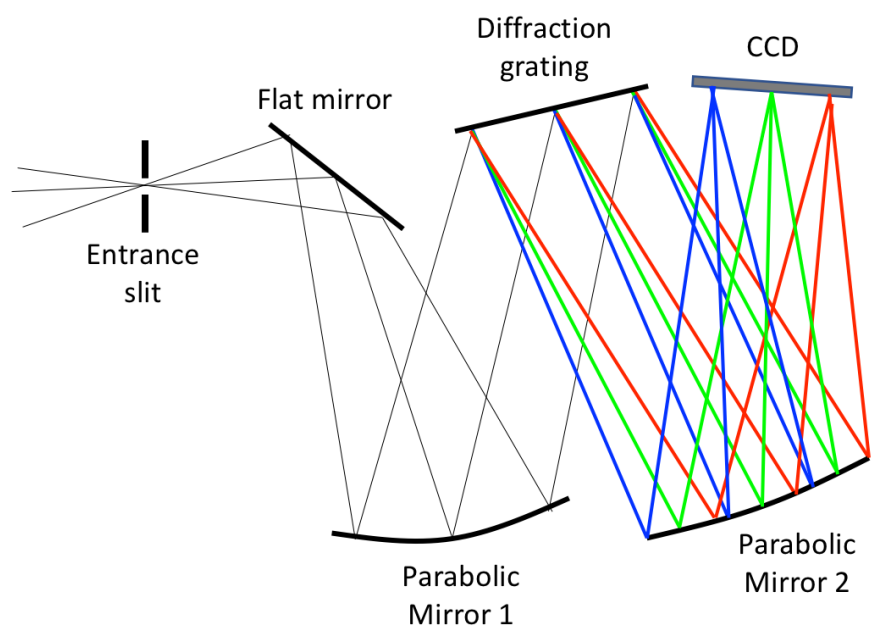

Figure 3.5: General layout of main optical components in a Czerny-Turner style Raman spectrometer.

The slit acts to restrict the angles of incoming light. This is important for peak resolution, as the variance in angle of incoming light will add a slight blur to the pure Raman peak. Thus, the narrower the entrance slit, the narrower the Raman peaks to the limit of the pure spectrum.

\subsubsection{Diffraction gratings}

A reflective diffraction grating works on the principle of inducing interference in light due to it interacting, either through reflection or transmission, with a periodic pattern 


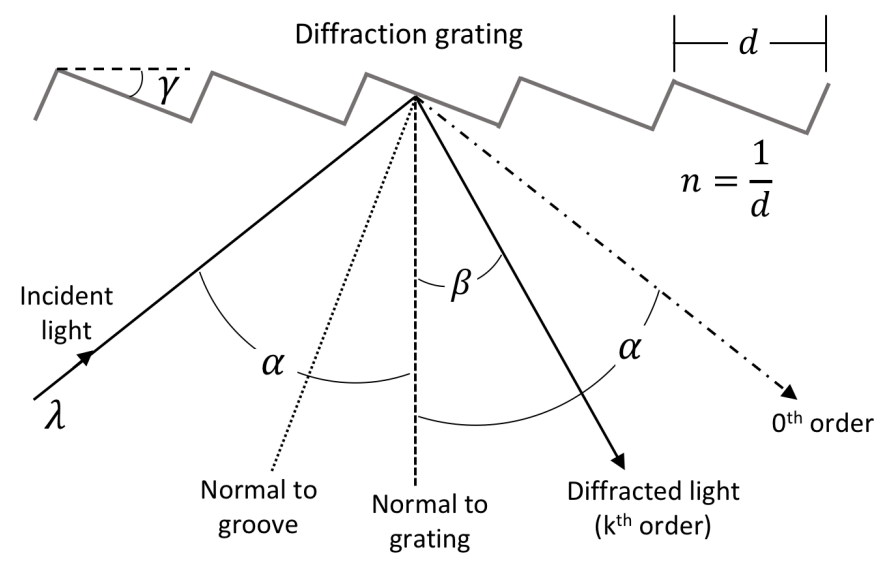

Figure 3.6: Illustration of blazed grating showing its grooves and the augmented light path that is induced.

on the grating surface. For a blazed grating, it is due to reflectance from grooves etched onto the grating surface. This leads to different wavelengths of light having different angles of reflection, governed by the equation

$$
\sin \alpha+\sin \beta=10^{-6} k n \lambda,
$$

where $\alpha$ is the angle of the incident light with respect to the grating norm, $\beta$ is the angle of the reflected light, $\lambda$ is the wavelength of the light, and $k$ and $n$ are the diffraction order and groove density of the grating, respectively. Figure 3.6 illustrates these grating and light path variables.

In Figure 3.6, the $0^{\text {th }}$ order reflected light path will be followed by light of any wavelength since it is the center of the interference pattern. When this path is centered on the CCD, the grating is said to be in zero-order mode, and simply acts as a mirror. The light from a higher order is what is used to produce a spectrum because the angle between the $0^{\text {th }}$ and higher orders, given by $\alpha-\beta$, differs for different $\lambda$. The blaze angle of the grating, given by $\gamma$, relates to the the other two angles via

$$
\alpha-\beta=2 \gamma
$$


The light reflected off of the diffraction grating is focused by a second parabolic mirror onto the CCD, over which the spectra will be dispersed. Linear dispersion is a measure defining the degree to which a spectral interval, in nm, is spread over the CCD in $\mathrm{mm} .{ }^{52}$ It is given by

$$
\frac{d \lambda}{d x}=\frac{10^{6} \cos \beta}{k n f}
$$

where $f$ is the focal length of the mirror focusing the light reflected off the grating onto the CCD, $x$ is length dispersed over in $\mathrm{mm}$, and $\beta, k$, and $n$ are the same as in Equation 3.16. Linear dispersion is specific to wavelength, since $\beta$ will not be the same over all wavelengths. A lower linear dispersion will thus provide a higher spectral resolution, and lower spectral range for a given CCD.

\subsubsection{Deep depletion CCD}

A charged-coupled device, or CCD, is an imaging device consisting of a 2-dimensional array of photosensitive pixels. A CCD consists of a semiconducting layer, typically made of doped silicon, below an array of electrodes. ${ }^{53}$ The silicon has a photoactive region, made so via doping, separated from the electrodes by a thin insulating layer. By applying voltages to the electrodes, a 2-dimensional array of potential wells (cells) forms throughout the photoactive layer. When electron hole pairs are induced in this layer, the electrons will migrate to the nearest cell. Over a given time interval, this build up of charge is proportional to the average intensity of light during that interval. To put it another way, the amount of charge built up in the cell will be proportional to the number of photons that were incident to the layer above. Pixel sizes typically range between $6-30 \mu \mathrm{m}$ across. ${ }^{51}$

After the time interval chosen for an image acquisition, the accumulated charge is read out by changing the voltage levels along each column. This causes the accumulated charge in one cell to shift to the one below, so that the columns of pixels 
are essentially shifting down towards the bottom edge of the CCD. Once one column value reaches the edge, it is collected into another series of cells, called the shift register which transfers the column horizontally in the same shifting manner to an amplifier. ${ }^{53}$ Many CCDs perform this entire process row-wise instead of column-wise. The amplifier produces a voltage proportional to the amount of charge run through it by a given cell, and this voltage is then digitized. The entire process is analog up until this final point. Light, and hence charge, still accumulates while this shifting is going on unless a shutter is used. This can produce vertical blur if the acquisition time is very short. For spectroscopic purposes, where full vertical binning (FVB) is often used, in which the total intensity value for a given column is the final information collected, this does not matter.

The normal configuration for consumer CCDs is referred to as front-facing, in which the electrodes sit on top of the photoactive silicon region, and attenuate some of the light. This reduces quantum efficiency (QE), which is the number of photoelectrons generated in the depletion region (doped region) per incident photon. For scientific purposes, where maximizing QE is worth a higher price, back-facing CCDs are used in which the photoactive region is facing outward and the electrodes are below this layer. This requires removing, either through chemical etching or some other technique, the bulk silicon substrate which is not photoactive and on which the photoactive region is grown.

For RS, and particularly RS in the NIR region, deep depletion CCDs are used, in which the doping is changed to increase the resistivity of the silicon. This allows for photons to be collected deeper within the photoactive region, which improves collection efficiency for NIR photons in the 700-1100 nm range. ${ }^{53,54}$ Such deep depletion CCDs require cooling to very low temperatures, such as $-85^{\circ} \mathrm{C}$, in order to reduce their higher dark current, which is thermal noise. This is typically achieved using a Peltier cooler, ${ }^{53}$ which is a thermoelectric cooling device, in which a temperature dif- 
ference forms between to different layers of conductor when a current is run through them. While not efficient, this form of cooling is highly reliable and can reach very low temperatures when vacuum insulated.

\subsubsection{Long-pass dichroic mirror}

Dichroic mirrors (DMs) are special mirrors that only reflect light above (or below) a certain wavelength, typically when placed at a $45^{\circ}$ angle. Those that reflect light shorter than a certain wavelength are called long-pass DMs because the longer wavelengths are allowed to transmit through the mirror, while those that reflect wavelengths longer than a certain wavelength are called short-pass DMs. In both cases, this selective reflectivity is produced by using multiple dielectric layers that are specifically spaced within the mirror to achieve the desired effect.

For Raman micro-spectroscopy, the high reflection and transmission efficiency of such mirrors, $95 \%+$ in relevant wavelength ranges, is useful to allow for the microscope objective to be used for illuminating the sample with the laser light and for collecting

the Raman scattered light. For example, using a long pass dichroic, the laser light is reflected downward through the objective and onto the sample. Of the reflected light returning up through the objective, the Rayleigh scattered light is reflected by the dichroic to the laser source, since it is the same wavelength, and the Raman scattered light, specifically the Stokes scattered light, is allowed to pass on towards the spectrometer.

\subsubsection{Microscope objective}

When Raman micro-spectroscopy is desired, that is collecting spectra from microscopic sample areas, the spectroscopic system must be coupled to a microscope ob- 


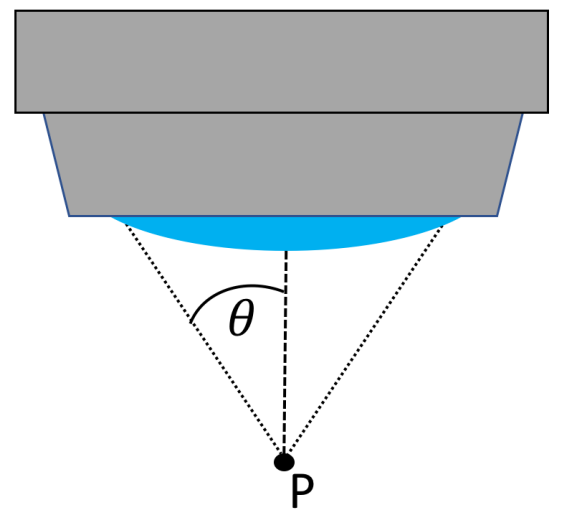

Figure 3.7: Microscope objective focusing on a point $\mathrm{P}$ directly below the pupil.

jective. One of the most important parameters defining a microscope objective is its numerical aperture (NA). The NA determines the maximum acceptance angle for light entering the objective from a point centred at its working distance. It is defined by

$$
\mathrm{NA}=n \sin \theta
$$

where $\theta$ is the maximum angle offset from the central axis, as seen in Figure 3.7, and $n$ is the index of refraction of the medium the light is passing through.

When regarding a laser coupled microscope objective, the rated NA of the microscope objective defines the maximum achievable when the beam diameter entering the rear pupil is maximized, since

$$
\mathrm{NA}=\frac{D_{\text {beam }}}{f_{\text {obj }}}
$$

where $D_{\text {beam }}$ is the diameter of the laser beam entering the objective. The maximum diameter entering is of course the diameter of the rear pupil. Since a propagating laser has a Gaussian cross section with respect to beam intensity, it is thus desirable to slightly overfill the rear pupil of the objective in order to avoid very low intensities around the rim. Having the maximum NA achievable for a given objective is desirable 
for minimizing laser spot size at the working distance of the objective, since spot size, defined as the diameter, $d$ of the laser spot, is inversely proportional to NA:

$$
d \geq \frac{1.22 \lambda}{N A}
$$

where $\lambda$ is the laser wavelength. ${ }^{55}$ The depth of field (DOF) of the focal volume of the laser spot is inversely proportional to $\mathrm{NA}^{2}$, and thus maximizing signal strength for a confocal setup requires maximizing NA.

Since NA is proportional to the refractive index of the working medium, a higher NA can be achieved by using an objective that can be immersed in a fluid denser than air, typically water or oil. A water immersion objective is desirable for biomedical applications of Raman spectroscopy because in vitro biological samples are stable in saline solutions that are similar to their natural in vivo environment, and water itself does not give off any measurable Raman spectrum. It also has a higher index of refraction than air, leading to a higher NA for the microscope objective. While oil immersion objectives can have an even higher NA, they have the drawbacks that oils have characteristic Raman peaks that contaminate the signal, and oil is not a natural solution for biological samples, particularly live samples. The reason a higher NA is desirable is that it allows the objective to get closer to the focal plane from which Raman spectra are being sampled, and thus to collect more of the light from that region.

\subsection{Multivariate statistical techniques}

There are numerous references that can be consulted for more information on the concepts covered in this section. Note that many statistical textbooks and papers use sums instead of means and scatters instead of variances for derivations where the 
divisor - the sample size - cancel out. For this work, the following references were used:

G. Cowan, Statistical data analysis, Oxford university press, 1998.

R. Gautam, S. Vanga, F. Ariese, and S. Umapathy, Review of multidimensional data processing approaches for Raman and infrared spectroscopy, EPJ Techniques and Instrumentation 2, 8 (2015).

A. J. Izenman, Modern Multivariate Statistical Techniques: Regression, Classification, and Manifold Learning, Springer, 2008.

I. Jolliffe, Principal Component Analysis, Springer, second edition, 2002.

M. Welling, Fisher Linear Discriminant Analysis, [Online] Available: https://www . ics.uci.edu/ welling/teaching/273ASpring09/Fisher-LDA .pdf [2018, June 10].

\subsubsection{Linear algebra and data representation}

For the purposes of data processing and statistical analysis, it is useful to consider a single Raman spectrum as a row vector, $\mathbf{x}=\left[x_{1}, x_{2}, \ldots, x_{m}\right]$, where the elements, $x_{1}, x_{2}, \ldots, x_{m}$, are scalar variables, being the intensity values of $\mathbf{x}$ at the first, second, and $m^{\text {th }}$ wavenumber respectively. Representing multivariate data in vector form is standard practice in multivariate statistics. A Raman spectrum is continuous before reaching the CCD, at which point it is binned into the discrete pixel columns of the sensor. This is why it can be represented by a finite vector of $n$ elements. Each bin is centred on some wavenumber, and the value of each $x_{i}$ is the measured intensity of the Raman spectrum at the wavenumber that pixel column is centred on.

Using this representation, a data set of $N$ Raman spectra is represented as a 
data matrix

$$
\mathbf{X}=\left[\begin{array}{c}
\mathbf{x}_{1} \\
\mathbf{x}_{2} \\
\vdots \\
\mathbf{x}_{N}
\end{array}\right]=\left[\begin{array}{cccc}
x_{1,1} & x_{1,2} & \ldots & x_{1, m} \\
x_{2,1} & x_{2,2} & \ldots & x_{2, m} \\
\vdots & \vdots & & \vdots \\
x_{N, 1} & x_{N, 2} & \ldots & x_{N, m}
\end{array}\right]
$$

where each spectrum $\mathbf{x}_{i}$ is a row in $\mathbf{X}$. With this representation, common statistical techniques such as finding the sample mean of data set $\mathbf{X}$, means finding the row vector $\mu_{\mathbf{X}}=\overline{\mathbf{X}}$ in which each element is the mean of each column in $\mathbf{X}$. The sample covariance matrix is given by

$$
\mathbf{C}=\frac{1}{N-1} \sum_{i=1}^{N}\left(\mathbf{x}_{\mathbf{i}}-\overline{\mathbf{X}}\right)^{\dagger}\left(\mathbf{x}_{\mathbf{i}}-\overline{\mathbf{X}}\right)
$$

From Equation 3.23 it can be seen that $\mathbf{C}$ is symmetric. Note that the diagonal elements of this matrix give the sample variance, $\sigma^{2}$ of each wavenumber, the square root of which is the sample standard deviation, and can be used to draw confidence intervals in a plot, with $\pm 2 \sigma$ being a $95 \%$ confidence interval for normally distributed variables. It should be mentioned that a dataset is usually mean centred before performing PCA and LDA, meaning the dataset mean $\overline{\mathbf{X}}$ is subtracted from each row of $\mathbf{X}$. If we write the mean centred data matrix as $\tilde{\mathbf{X}}$, this leads to a simplified expression for covariance of

$$
\mathbf{C}=\frac{\tilde{\mathbf{X}}^{\dagger} \tilde{\mathbf{X}}}{N-1}
$$

\subsubsection{Principal component analysis}

Principal component analysis (PCA), is a technique for dimensionality reduction which reduces a dataset into one of uncorrelated variables, ordered by contribution to total variance. ${ }^{56}$ Two variables are said to be correlated if their Pearson correlation 
coefficient, given by

$$
\rho=\frac{\operatorname{Cov}(x, y)}{\sigma_{x} \sigma_{y}}
$$

is non-zero. In Equation 3.25, $\operatorname{Cov}(x, y)$ is the covariance of variables $x$ and $y$, while $\sigma_{x}$ and $\sigma_{y}$ are their corresponding variances. It follows that two variables that have zero covariance must be uncorrelated. For Raman spectra, where we consider each wavenumber a variable, Equation 3.25 would thus correspond to the correlation between two wavenumbers. Since the FWHM of Raman peaks can be seven wavenumbers wide or more, and since molecules often have more than one characteristic Raman peak in the wavenumber range being examined, it follows that Raman spectra variables are highly correlated. This is good since it means there is redundancy in the data and its dimensionality can thus be reduced without losing important information.

PCA works by finding a linearly independent basis for the dataset, and transforming the data to this new basis. In terms of linear algebra, it performs an orthogonal transformation from the initial basis, which for Raman spectra is in wavenumber space, to this new one. This is done by finding the eigenvectors of the covariance matrix of the dataset, sorted by eignenvalue. That is, for the covariance matrix $\mathbf{C}$ of the entire data set, with PCA we wish to find the vectors $\mathbf{v}$ such that

$$
\mathbf{C v}=\lambda \mathbf{v}
$$

where $\lambda$ is an eigenvalue of $\mathbf{C}$, and corresponds to the variance of $\mathbf{v}$ in the transformed feature space. To do this, one must diagonalize $\mathbf{C}$, by finding the orthogonal matrix V such that

$$
\mathbf{V}^{\dagger} \mathbf{C V}=\mathbf{D}=\left[\begin{array}{ccccc}
\lambda_{1} & 0 & 0 & \ldots & 0 \\
0 & \lambda_{2} & 0 & \ldots & 0 \\
\vdots & \vdots & \vdots & \ddots & \vdots \\
0 & 0 & 0 & \ldots & \lambda_{n}
\end{array}\right]
$$



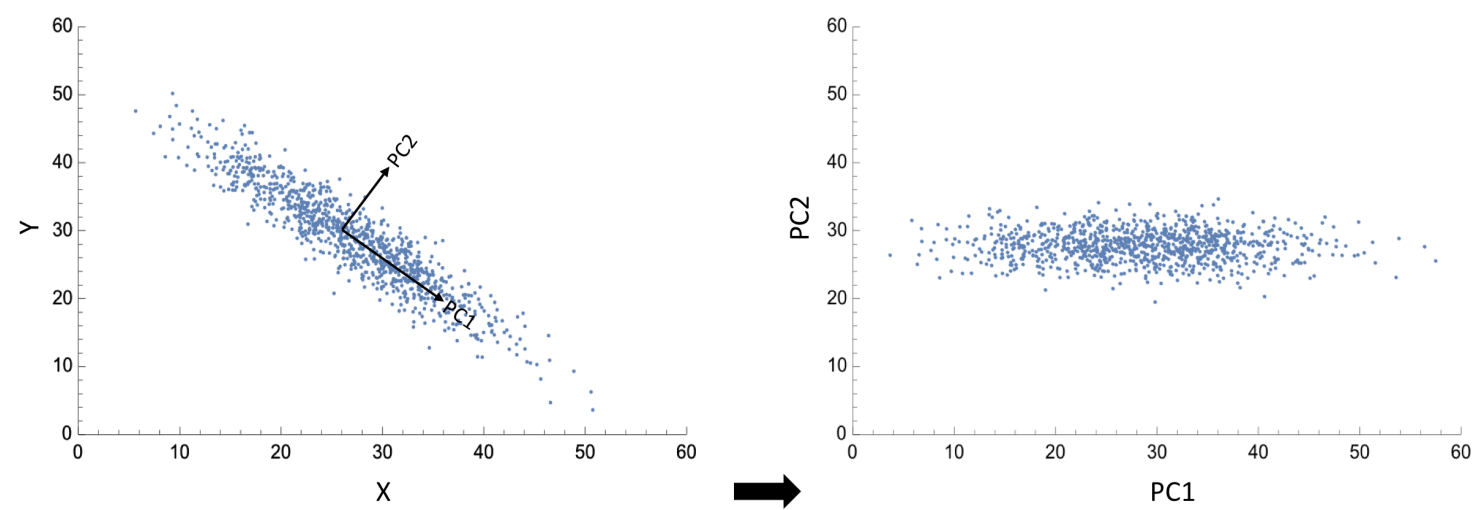

Figure 3.8: PCA example in two dimensions. Data are rotated about mean into new basis of uncorrelated PCs.

where $\mathbf{D}$ is a diagonal matrix where each $\lambda_{i}$ is an eigenvalue of $\mathbf{C}$ and the corresponding column $\mathbf{v}_{i}$ in $\mathbf{V}$ is an eigenvector. $\mathbf{V}$ is chosen such that each $\mathbf{v}_{i}$ is a unit vector. These eigenvectors are our principal components (PCs). We then project our original data set $\mathbf{X}$ into this new basis

$$
\mathbf{X}_{P C}=\mathbf{X V}
$$

The covariance matrix of $\mathbf{X}_{P C}$ is then simply $\mathbf{D}$, meaning the covariance between different PCs is zero. Thus, we have found a set of uncorrelated variables. Geometrically, this is equivalent to rotating the data around its mean such that there is no correlation between variables. This is visualized by Fig 3.8, in which a two variable data set is rotated around its mean value. Note that the data are clearly spread out more along PC1 because PCs are ordered by variance $(\lambda)$.

By choosing a subset of principal components, in order of contribution to total variance (i.e. by descending $\lambda$ ), one can choose a subset of features which is much smaller than the original number of features in a spectrum, and subsequently smaller than or at most equal to the total number of samples. This is statistically desirable due to the curse of dimensionality, in which is always possible to find linear separation between $\mathrm{N}$ data points in a feature space of dimension $d \geq N$. This is also 

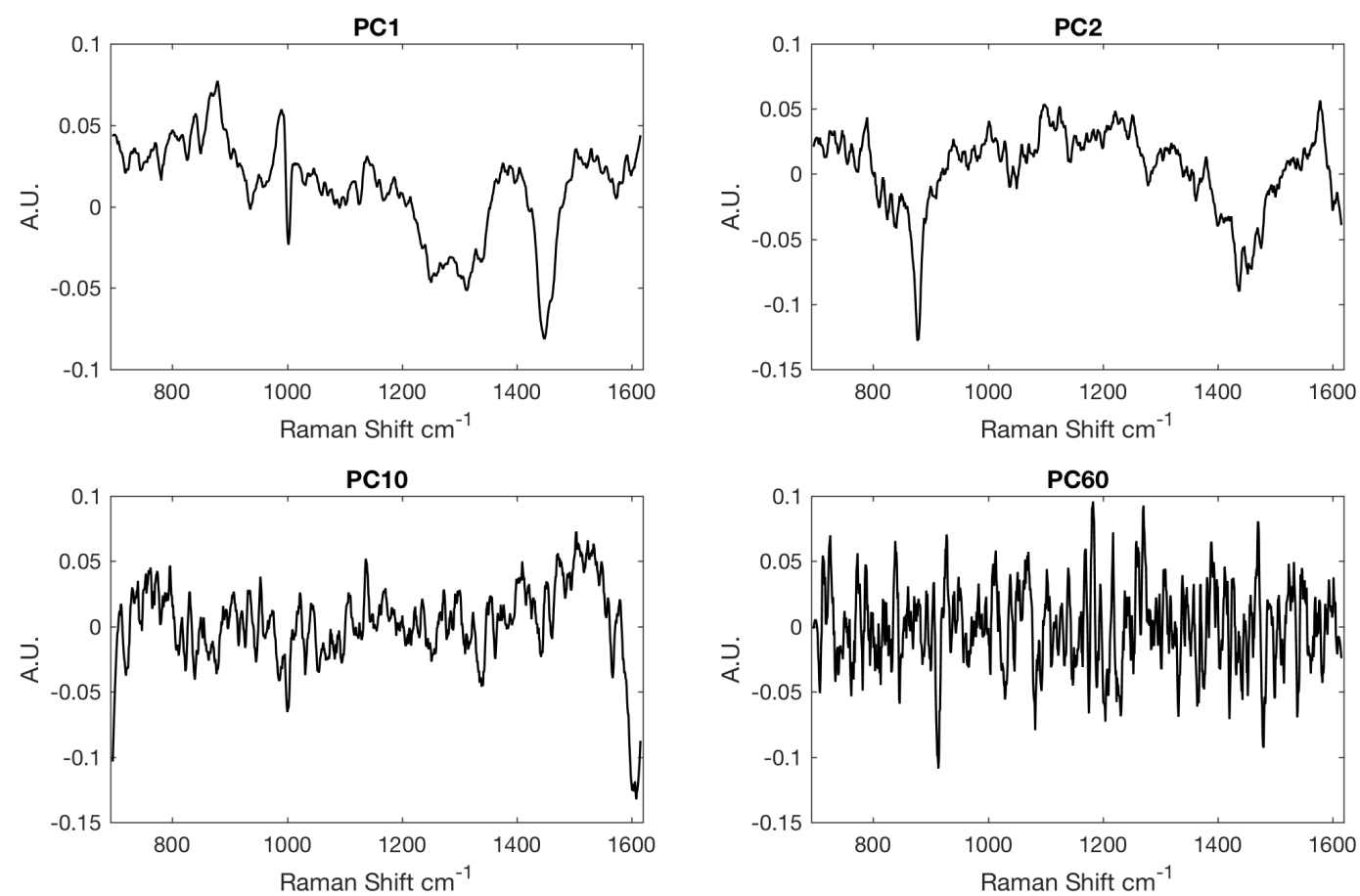

Figure 3.9: Comparison between low PCs, which have a larger contribution to variance, to higher ones.

useful because usually higher PCs with lower contribution to total variance typically correspond to noise rather than differences between subclasses within the data. This is demonstrated in Figure 3.9 where the first 2 PCs from the HLE data as well as the $10^{\text {th }}$ and $60^{\text {th }}$ are shown.

As can be seen, the lower PCs have some structure to them, while the higher PCs appear more random (noisy).

\subsubsection{Linear Discriminant Analysis}

Linear discriminant analysis (LDA) is a method for projecting a high dimensional dataset with $N$ subclasses onto a lower, $N-1$ dimensional hyperplane which maximizes the distance between class means while minimizing the within class variance. In the binary case, when there are only two classes, this means the data set is projected 


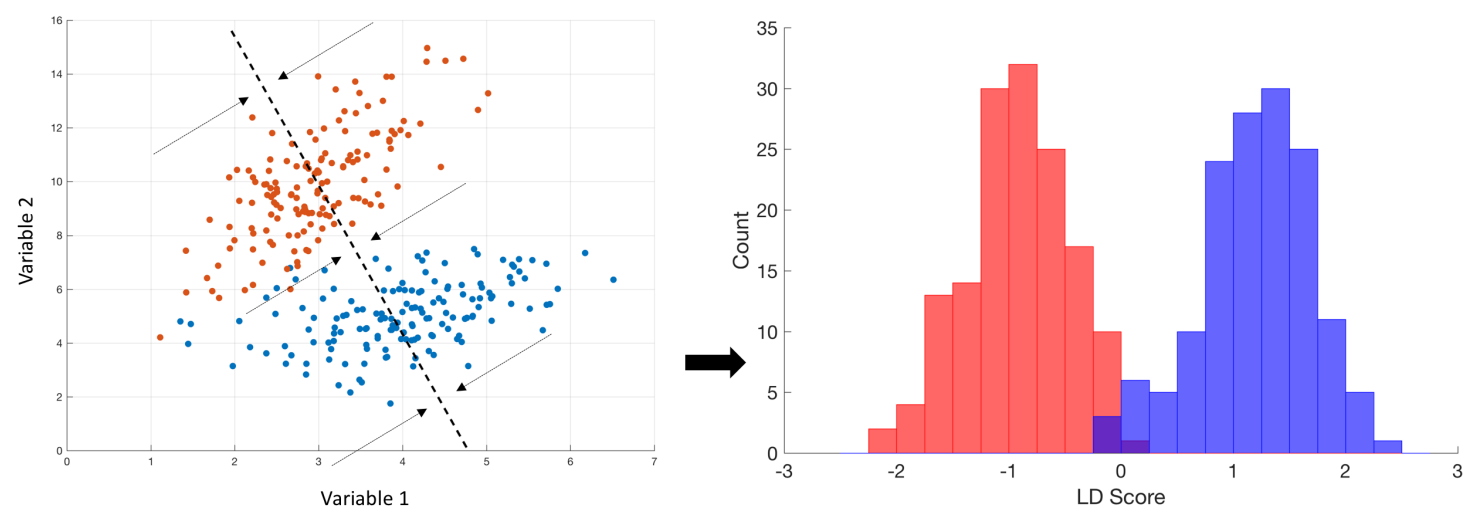

Figure 3.10: Visualization of two class distribution being projected onto a linear discriminant which best separates them. The data points are projected onto the linear discriminant (dashed line), and binned by LD score, thus generating the histogram on the right for which classification can be done by choosing an optimal threshold and setting it to zero. The red data points on the scatter plot correspond to the red distribution on the LD score histogram, and the blue data points correspond to the blue distribution. The dark purple section of the histogram represents overlap between the two distributions.

onto a line which maximizes class separation if the classes are linearly separable. It can thus be used for binary classification of linearly separable datasets. A visualization of this is shown in Figure 3.10.

In this example a 2 dimensional dataset with two classes is projected onto a line which best separates the classes, and then a point is found which maximizes either classification accuracy or sensitivity or specificity, depending on what is desired. Because class labels must be known to perform LDA, it is referred to as a supervised technique.

Mathematically, binary LDA involves finding a linear discriminant (LD), a, which is a unit vector used to project each spectrum, $\mathbf{x}$, onto the line that passes 
through a:

$$
s=\mathbf{x} \cdot \mathbf{a}=\left[x_{1} x_{2} \ldots x_{n}\right]\left[\begin{array}{c}
a_{1} \\
a_{2} \\
\vdots \\
a_{n}
\end{array}\right]
$$

The scalar result, $\mathrm{s}$, is called the $L D$ score of $\mathbf{x}$, and the values $a_{1}, a_{2}, \ldots, a_{n}$ are the called the linear discriminant loadings (LD loadings). An an entire dataset can then be projected onto the line via

$$
\mathbf{s}=\mathbf{X} \mathbf{a}
$$

where $\mathbf{s}$ is a vector containing the LD score for every spectrum in the dataset. In order to find an LD that optimizes between class separation and minimize within class variance, a is chosen such that the following function is maximized

$$
\begin{aligned}
J(\mathbf{a}) & =\frac{\left(\boldsymbol{\mu}_{1} \mathbf{a}-\boldsymbol{\mu}_{2} \mathbf{a}\right)^{2}}{\mathbf{a}^{\dagger} \mathbf{C}_{1} \mathbf{a}+\mathbf{a}^{\dagger} \mathbf{C}_{2} \mathbf{a}} \\
& =\frac{\mathbf{a}^{\dagger} \mathbf{B a}}{\mathbf{a}^{\dagger} \mathbf{W a}},
\end{aligned}
$$

where $\mathbf{W}=\mathbf{C}_{1}+\mathbf{C}_{2}$ is the sum of the covariance matrices of each class, $\boldsymbol{\mu}_{\mathbf{1}}$ and $\boldsymbol{\mu}_{\mathbf{2}}$ are the mean spectra for the two classes, and the matrix $\mathbf{B}$ is defined by $B_{i j}=$ $\left(\mu_{1}-\mu_{2}\right)_{i}\left(\mu_{1}-\mu_{2}\right)_{j} .{ }^{57}$ Note that, because a acts linearly on the dataset, the mean LD score is the LD score of the mean, and so $\mathbf{a} \mu_{1}$ is the mean LD score of class 1 and $\mathbf{a}^{\dagger} \mathbf{C}_{1} \mathbf{a}$ is the variance. A proof is available in Appendix A.2. It can be shown that the measure, $J$, is maximized when

$$
\mathbf{a} \propto \mathbf{W}^{-1}\left|\boldsymbol{\mu}_{1}-\boldsymbol{\mu}_{2}\right|
$$

Since this a proportionality relation, a discriminant of any magnitude in the above direction in wavenumber space can be used, and so typically the LD is chosen to be 
unity so that the dot product is merely a projection. Thus, to find the LD, one must find the class means and class covariance matrices, then estimate the inverse matrix of the matrix $\mathbf{W}$, which is the sum of these matrices. If the dimensionality of the dataset is greater than the number of samples, the covariance matrices, and their sum, will often be singular and the processes of estimating the inverse of $\mathbf{W}$ will fail. This is a large part of why the initial PCA dimensionality reduction step is necessary.

Once the training data has been projected onto the LD, a threshold score is determined that divides the scores between the two classes optimally, where the optimum is user determined. In this study the threshold was chosen to maximize accuracy, which is the ratio of correctly classified data points over the total number of data points.

\subsubsection{Cross-validation}

In order to estimate the performance of a classifier, a cross-validation scheme, in which a dataset is divided into a training and a test set is used. This can be done by choosing a randomly selected subset, e.g. 25\%, to be used as the test set, with the remainder used as the training set to train the classifier. The training and test sets are typically chosen to be stratified over the classes, so that there the number chosen per class is proportional to that class relative to the entire dataset. This is then repeated several, preferably thousands, of times, and the average accuracy, with error, can then be given.

Another method is to choose the subsequent test sets to be disjoint, choosing a test set size $m$, that divides into the total dataset size $N$. This will then give $k=N / m$ cross-validation with test sets that share no element, and is thus called $\mathrm{k}$-fold cross-validation. This is faster than the above method for comparable test set sizes, since one is limited to $N / m$ runs, while the randomly selected test set method 
can be performed, assuming a test set which is stratified over the classes, a maximum of $(p N) ! /(p m !(p N-p m) !)$, where $p$ is the fraction of the dataset that belongs to the smallest class. For a binary classifier with equal class sizes, $p=0.5$. As $\mathrm{N}$ increases, this quickly becomes computationally infeasible.

Leave-one-out cross-validation (LOOCV) is a variant of $\mathrm{k}$-fold cross-validation in which $m=1$ and so $k=N-1$. In this case, only one datapoint is left out for testing, and so the classifier is trained on almost the entire dataset. This is done $N$ times, with each datapoint being tested. While this is more computationally expensive than using smaller values for $k$, it gives an unbiased estimate of classifier performance. It does, however, have the highest variance for the estimate, since each test is either $100 \%$ or $0 \%$ accurate, depending on how the left out datapoint is classified. The variance is thus given by

$$
\sigma^{2}=\frac{A(1-A)}{1-1 / N}
$$

where $\mathrm{A}$ is the mean accuracy (see Appendix A.3). The standard deviation, $\sigma$, is the square root of this, and standard deviation of the mean, which would be the error reported on any LOOCV accuracy estimate, is $\sigma_{m}=\sigma / \sqrt{N}$. Thus,

$$
\sigma_{m}=\sqrt{\frac{A-A^{2}}{N-1}}
$$

In all cross-validation methods, a confusion matrix can be generated, the layout 
of which is shown below:

\section{Actual}

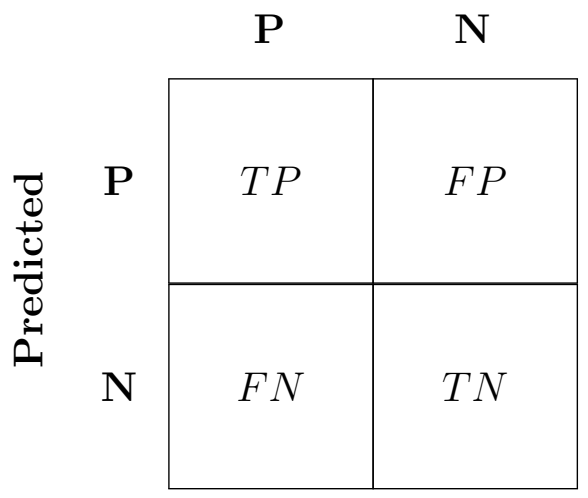

where TP, TN, FP, and FN are true positive, true negative, false positive, and false negative counts respectively. TP is the number of positive samples classified as such, TN are the number of negative samples classified as such, FP are the number of negative samples classified as true, and $\mathrm{FN}$ are the number of positive samples identified as negative. Using the scheme, for $\mathrm{N}$ total samples, the accuracy of a classification is the fraction of samples correctly classified in total, and so is equal to (TP $+\mathrm{TN}) /(\mathrm{TP}$ $+\mathrm{FP}+\mathrm{FN}+\mathrm{TN})$. The sensitivity is fraction of positive samples identified correctly as positive, and thus equal to $\mathrm{TP} /(\mathrm{TP}+\mathrm{FN})$, and the specificity is fraction of negative samples correctly classified as such, equivalent to $\mathrm{TN} /(\mathrm{TN}+\mathrm{FP})$. 


\section{Chapter 4}

\section{Materials and methods}

This chapter covers how the experiment was performed, and the instrumentation used. Included is a summary of the main apparatus, the custom-built Carleton Raman Microscope, including performance characteristics relating to its various parts; information regarding how the HLE cells were grown, irradiated, stored, and analyzed via Raman spectroscopy; and a description of the algorithms used to preprocess the data and analyze it. The theoretical aspects of some of the methods, such as PCA and LDA, were described in the previous chapter, with only their application described here.

\subsection{Carleton Raman Microscope}

Raman spectra were recorded using the Carleton Raman Microscope located in Herzberg Laboratories. This microscope was built by former students, Hamid Moradi and Dean Shepherdson, in the Murugkar lab. ${ }^{58}$. Since an upgraded version was later created and is discussed in this thesis, the original version of the CRM used in this experiment will hereby be referred to as CRM1. A schematic of CRM1 is given in Figure 4.1 and 
a photograph of the apparatus can be found in section C.2 of the appendix. CRM1 consists of a near-infrared continuous-wave (cw) laser, a cage system used for guiding the beam through the necessary optical elements, such as the microscope objective, and guiding the signal to a spectrometer for receiving the Raman scattered light. The sample sits on a motorized 3-axis stage, and monochrome camera is connected to the system to enable bright-field imaging. The $785 \mathrm{~nm} \mathrm{cw}$ laser emits a collimated $2 \mathrm{~mm}$ diameter beam which is expanded and further collimated by lenses L1 and L2, which have $f_{1}=-100 \mathrm{~mm}$ and $f_{2}=40 \mathrm{~mm}$ focal lengths respectively. The beam expansion is necessary to allow for a slight overfill of the $8 \mathrm{~mm}$ back-aperture of the microscope objective L3. After being guided by a few mirrors, the laser beam is then reflected downward by a long-pass dichroic mirror (DM), through the objective and focused onto an approximately 1 micron spot on the sample. Both Rayleigh and Raman scattered light, as well as fluorescent light, are transmitted back up through the objective towards the DM. The laser light is again reflected by the DM back down the path it had come, while the Raman signal is allowed to pass through, since it is primarily Stokes scattered Raman photons and thus has a longer wavelength than the laser light.

The Raman signal passes through an long-pass edge filter (Iridian Spectral Technologies, Ottawa, Canada) which further filters out any remnant laser light. It is then guided by more mirrors towards a lens (L4) that focuses it through a $100 \mu \mathrm{m}$ pinhole, which provides confocality to the setup, as shown in Figure 4.2. A confocal microscope acquires light from a finite focal volume, with light from shallower and deeper focal depths being filtered. This helps reduce the contribution of quartz fluorescence to the signal from the quartz sitting below each cell. It also allows for targeting single point volumes with respect to all three dimensions. The signal then passes through another pair of lenses (L5), which correct for the dispersion angle so as to match the numerical aperture (NA) of the Andor spectrometer. After entering the 


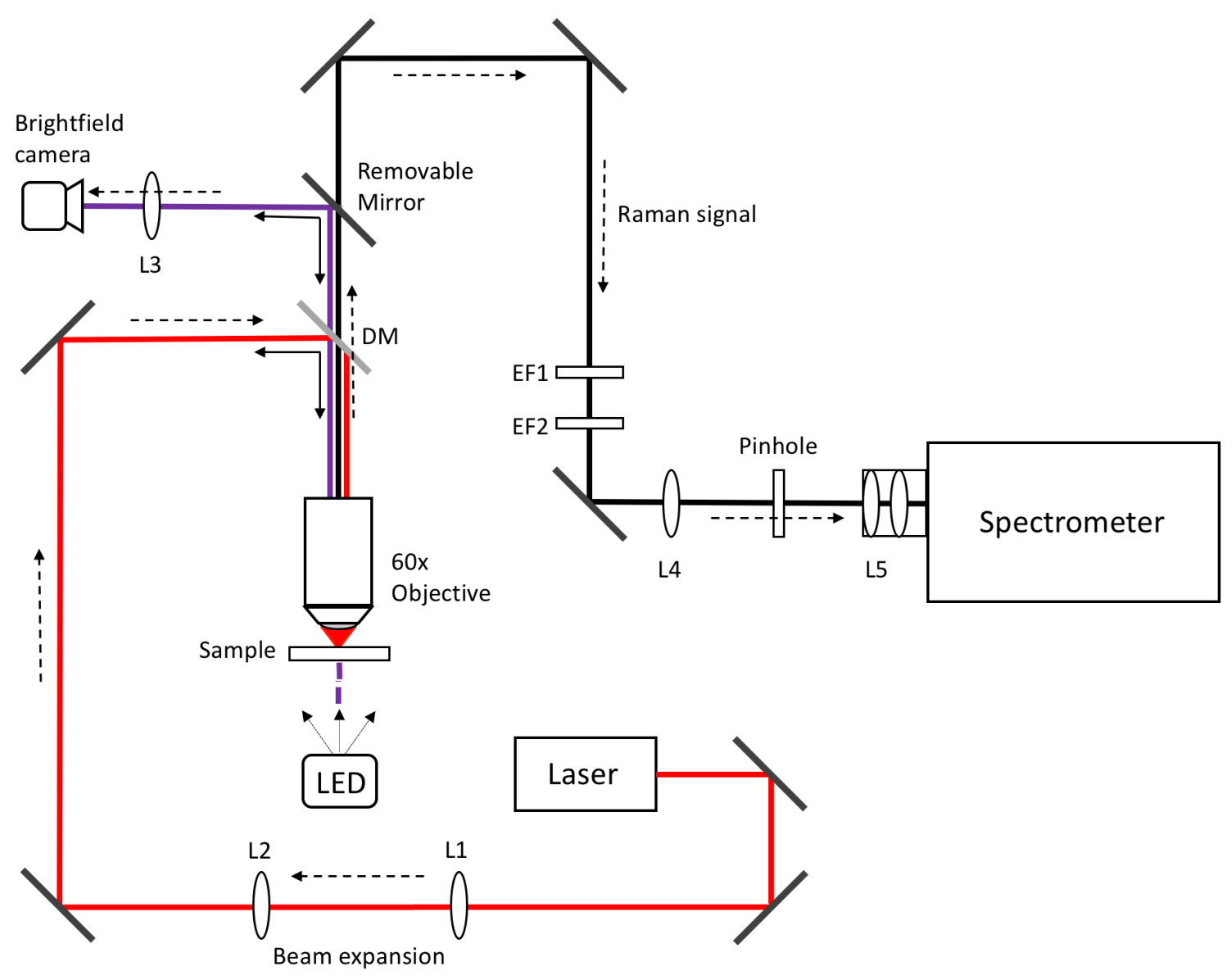

Figure 4.1: Schematic of the Carleton Raman Microscope version 1 (CRM1) used for this experiment. It consists of the following components: a $785 \mathrm{~nm} \mathrm{cw}$ laser with $100 \mathrm{~mW}$ output and $2 \mathrm{~mm}$ beam diameter, L1: $-100 \mathrm{~mm}$ focal length planoconvex lens, L2: $40 \mathrm{~mm}$ focal length plan-convex lens, DM: long-pass dichroic mirror for $>785 \mathrm{~nm}, 60 \mathrm{x}$ water immersion microscope objective, removable mirror - put in place to acquire brightfield and removed when acquiring spectral data, L3: $100 \mathrm{~mm}$ focal length biconvex lens, brightfield camera with monochrome CCD with 1280x1024 resolution, EF1 and EF2: long-pass edge filters, L4: $100 \mathrm{~mm}$ focal length plano-convex lens, pinhole with $100 \mathrm{\mu m}$ diameter, L5 double lens system for optimizing dispersion angle of light entering spectrometer with focal lengths $\mathrm{f} 1=40 \mathrm{~mm}$ and $\mathrm{f} 2=100 \mathrm{~mm}$, spectrometer with a $900 \mathrm{~nm}$ blaze diffraction grating (1000 lines/mm groove density) and a 1024 pixel horizontal resolution deep depletion CCD. Three-axis motorized stage not shown. 


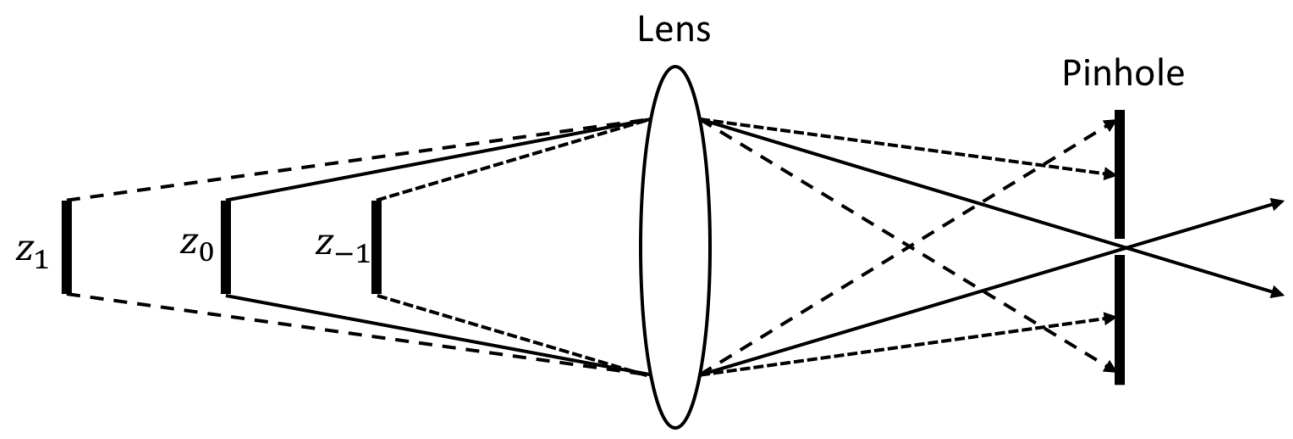

Figure 4.2: Visualization of light ray paths through a pinhole with paths corresponding to different focal depths filtered.

spectrometer and being reflected off a mirror, then off the diffraction grating, then off another mirror and onto the main CCD, the signal is recorded and transmitted via USB to a PC running Andor SOLIS acquisition software (Andor Technology, Belfast, Northern Ireland). Brightfield images were acquired using a 1280x1024 monochrome CCD camera (Thorlabs, Newton, New Jersey, USA).

The microscope objective used was a LUMFLN 60x water immersion objective (Olympus, Tokyo, Japan) with a 1.1 numerical aperture (NA). It has a rear pupil diameter of $8 \mathrm{~mm}$, and a $1.5 \mathrm{~mm}$ working distance. The DM used was of the longpass variety. It was a Semrock Razer Edge long-pass DM (Semrock, Rochester, NY, USA) that reflects wavelengths at $785 \mathrm{~nm}$ and below to $>95 \%$ efficiency. The threeaxis motorized stage was an ASI MS-2000 (ASI, Eugene, OR, USA) with $22 \mathrm{~nm}$ step resolution and $<700 \mathrm{~nm}$ RMS error on repeatability.

\subsubsection{Laser}

The laser used was a $785 \mathrm{~nm}$, continuous-wave (cw) diode laser (Innovative Photonic Solutions, Monmouth Junction, NJ, USA), with min power of $100 \mathrm{~mW}$. Its output spot size is $2 \mathrm{~mm}$ and its rated stability is up to $5 \%$ fluctuation. It was coupled to a ThorLabs power controller. The beam quality factor is rated $M^{2}<1.5$, where 
$M^{2}=1$ would indicate an ideal, collimated Gaussian intensity profile, allowing for optics to reach their diffraction limit for the given wavelength. This value is close enough to provide an approximately diffraction limited spot size.

Using a $785 \mathrm{~nm}$ wavelength laser is important as it is an ideal wavelength for probing biological materials. It induces minimal fluorescence in such materials, and the near-infrared (NIR) photons do not cause photo-damage for intensities in the 0-15 $\mathrm{MW} \mathrm{cm}^{-1}$ range, as irradiating human cells with $785 \mathrm{~nm}$ laser light at $1 \mu \mathrm{m}$ spatial resolution and $115 \mathrm{~mW}$ of power for 40 minutes was performed by Notingher et al, and no change to the Raman spectra or other signs of photodamage were found. ${ }^{59}$ The same could not be said for the shorter wavelengths tested in that study. A $785 \mathrm{~nm}$ laser is not too long a wavelength to produce a Stokes-scattered Raman shift in the fingerprint region of $500-1500 \mathrm{~cm}^{-1}$. These Raman shifts, unfortunately, are even deeper in the NIR range, making them more difficult to detect. Using a deepdepletion CCD image sensor and longer acquisition times can make up for this. In the end, the choice of wavelength is a compromise between maintaining sample and signal integrity with signal strength, and since signal strength can be improved in other ways, it is a less critical criterion.

\subsubsection{Andor spectrometer}

The spectrometer used to acquire Raman spectra was a Shamrock SR-303i (Andor Technology, Belfast, Northern Ireland) with Czerny-Turner configuration. It features a back-illuminated deep depletion CCD (Newton DU920 BEX2-DD, Andor Technology, Belfast, Northern Ireland) with an anti-fringing, extended range dual AR coating, which is cooled thermoelectrically, to a temperature of $-85^{\circ} \mathrm{C}$ for this study. Its resolution is $1024 \times 256$, over a $26.7 \times 6.7 \mathrm{~mm}$ imaging area. Diffraction gratings are mounted on a 3-sided rotatable turret, giving a maximum of 3 gratings per turret. 
The grating facing the mirrors is the one in use, and switching gratings in the Andor SOLIS software, with which the spectrometer communicates, leads to the turret rotating into the correct position so that the chosen grating is facing the mirrors. The diffraction grating used had 1000 lines/mm groove density, and $900 \mathrm{~nm}$ blaze. This was optimal for the spectral range of $830.37-890 \mathrm{~nm}$ used in this study.

\subsection{System performance}

The laser power below the microscope objective was approximately $30 \mathrm{~mW}$. The laser spot size is taken as the spot diameter, $d=2 r$, where $r$ is the radius and is estimated by the approximation $r=0.61 \lambda / N A$, where $\lambda=785 \mathrm{~nm}$ is the laser wavelength. This gives a radius of $0.435 \mu \mathrm{m}$ for a spot size of $d=0.87 \mu \mathrm{m}$, or approximately $0.9 \mathrm{~mm}$. Using this value for spot size, we can estimate the intensity at the sample as being approximately $4 \times 10^{6} \mathrm{~W} / \mathrm{cm}^{2}$. This is just a rough estimate, and is calculated assuming a uniform spot intensity, rather than the actual Gaussian distribution, in which power density would vary over the spot.

The confocal resolution, which is the axial resolution, is taken as the depth of field, which can be theoretically found using

$$
D O F=n \lambda / \mathrm{NA}^{2}
$$

where $n$ is the refractive index of the immersion medium. For HLE cells immersed in PBS, which should have a similar refractive index to water at $n=1.33$, this gives a theoretical DOF of approximately $0.86 \mu \mathrm{m}$. The actual measured axial resolution was found to be closer to $5 \mu \mathrm{m}$. Using this value, and approximating the volume shape as a cylinder, gives a volumetric power density of $9.4 \mathrm{~mW} / \mathrm{\mu m}^{3}$. 


\subsubsection{Silicon and Polystyrene}

Silicon and polystyrene samples are used to calibrate our instrument and determine instrument performance. Since these materials are relatively pure and reusable, and the spectra they produce vary little with respect to where on the sample they are acquired, they are ideal for this purpose and for determining the performance of our apparatus. The spectrum of both sample types are shown in Figure 3.4. The silicon spectrum is dominated by a single prominent peak, centred at $520 \mathrm{~cm}^{-1}$, and for $1 \mathrm{~s}$ acquisition time, the CRM1 typically found a peak height of about $32 \times 10^{3}$ counts. For polystyrene, there are several peaks, but the peak centred at $1001.4 \mathrm{~cm}^{-1}$ is by far the largest. Using a $15 \mathrm{~s}$ acquisition time, the CRM1 typically attained peak heights of $30 \times 10^{3}$ counts for this sample type. Note that while the Andor SOLIS spectroscopic software gives peak intensities as counts, the spectrometer is not photon counting and these values should be considered as relative intensities, proportional to the true signal intensity.

\subsection{Sample preparation}

A batch of HLE cells is any set of cells grown together on multiple circular quartz cover slips, one for each dose. For each batch of HLE cells grown in this experiment, either 7 or 5 quartz cover slips, depending on whether the ultra-low doses (0.01 Gy and 0.05 Gy) were included, were seeded with cells at Health Canada and allowed to propagate for 24 hours before irradiation. Seven slips were used in cases where ultralow dose samples were included. Ultra-low doses were 0.01 Gy and 0.05 Gy, while $0.25,0.5,2$, and 5 Gy made up the remainder, with a 0 Gy control included in each batch. Measurements were made on 7 batches in total. Some of the quartz slips used had high fluorescence, which decreased the signal-to-noise ratio substantially, and 
thus these samples were not included in the binary discrimination, to avoid skewing classification results.

\subsubsection{Seeding HLE cells}

The HLE cells used were a transformed (SV-40 virus) cell line, acquired from ATCC (Manassas, VA, USA). The SV-40 virus is an immortality virus that prevents cells from leaving the cell cycle, and in the case of HLE cells, from differentiating into LF cells. The cells were incubated at $37^{\circ} \mathrm{C}$, with a $5 \% \mathrm{CO}_{2} / 95 \%$ air atmosphere and 95\% humidity. They were grown in $75 \mathrm{~cm}^{2}$ tissue culture flasks with Eagles minimum essential medium (EMEM) with $20 \%$ fetal bovine serum. After growing to confluence ( $\sim 10^{6}$ cells) over $2-3$ days, the cells were seeded onto $18 \mathrm{~mm}$ diameter, $250 \mu \mathrm{m}$ thick quartz coverslips (Tedpella, Redding, CA, USA) in $3 \mathrm{ml}$ of medium. This was done the day before the exposure. On the day of exposure, these coverslips were placed in a new dish, again with $3 \mathrm{ml}$ of media, but also with 100 units $\mathrm{ml}^{-1}$ of penicillin and $100 \mu \mathrm{g} \mathrm{ml}^{-1}$ of streptomycin (Invitrogen Canada Inc. Burlington, ON, Canada). Only adhered cells remained on the quartz coverslip after this point.

\subsubsection{Irradiating cells}

Twenty-four hours after seeding, the quartz coverslips with adhered HLE cells were irradiated with $120 \mathrm{kVp}, 12.5 \mathrm{~mA}$ x rays using a cabinet x-ray machine (X-RAD 320, Precision X-Ray Inc., North Branford, CT, USA) and a $2 \mathrm{~mm}$ thick aluminum filter. A dose rate of 38.2 cGy per minute was used at sample position. This rate was determined using a Radcal 9010 ion chamber (Radcal, Monrovia, CA) calibrated at the National Research Council, Ottawa, ON. The sample position was $50 \mathrm{~cm}$ from the X-ray tube. Samples were irradiated to 0, 0.01, 0.05, 0.25, 0.5, 2, and 5 Gy doses. The ultra-low doses of 0.01 Gy and 0.05 Gy were only performed on two of the four 
batches. The samples were then incubated at $37^{\circ} \mathrm{C}$ for 24 hours after exposure, and then fixed using a solution of $4 \%$ formaldehyde. After washing with PBS three times, the samples were placed in petri dishes with a small amount of PBS. The dishes sealed using parafilm to avoid leaking during transport. The work of seeding and irradiating the cells was performed by Dr. Vinita Chauhan at Health Canada, Consumer and Clinical Radiation Protection Bureau, Ottawa, ON.

\subsection{Data acquisition}

The HLE cells were then transported to Herzberg laboratories at Carleton University where they were kept in a biosafety cabinet to prevent contamination. Each day, one of the samples for a given dose was opened, and immersed in extra phosphate-buffer saline (PBS) to allow for immersion of the microscope objective. This sample was then taken to the CRM and as many nucleus and cytoplasm measurements as possible were made. In some instances, measurements had to be rejected and replacement measurements made on different cells within the same sample due to the occasional bacterial cell getting into the laser spot, but this was not common. This was determined to have happened if a bacterium was seen, indicated by its small size and brownian motion, near the acquisition location after finishing an acquisition. Usually such measurements were stopped mid-acquisition due to the appearance of a highly unusual looking Raman spectrum. Quickly acquiring the spectrum of the nearby suspected bacterium quickly showed it to be the cause. Even when no clear changes in the spectrum were visible, spectra were rejected if any suspected bacterium was seen within approximately $25 \mu \mathrm{m}$ of any edge of the $3 \times 3$ grid. Such instances occurred rarely, during the last few measurements of the day, when the sample had been exposed to laboratory air for several hours.

Spectra from each cell were acquired from a 3x3 grid of 9 spots, with the average 
of these 9 spots taken as the spectrum for that cell. This was done for both the nucleus and cytoplasm. For the nucleus, the spacing between the $1 \mu \mathrm{m}$ spots was $3 \mu \mathrm{m}$, make the total grid dimensions $6 \mu \mathrm{m}$ by $6 \mu \mathrm{m}$. For cytoplasm, finding an area that could fit such dimensions was more difficult, and so $1 \mu \mathrm{m}$ spacing between points was used instead. This was because the parts of the HLE cells outside the nucleus tended to stretch out in tapering strands, over which the larger grid size usually did not fit.The acquisition time was 1 minute per spot, for a total acquisition time of 9 minutes. The 1 minute spectrum from each spot was the average of three 20 s spectra. This was necessary to allow for cosmic ray removal. The cosmic ray removal routine in Andor SOLIS's scripting software was used for this. It compared all three frames, and if one had an extreme outlier peak, as cosmic rays cause, it would replace it with a value from one of the other frames. This was done such that a cosmic ray in a frame would be replaced by the intensity at that wavenumber found in the previous frame, except for the first frame which used the value from the third.

The script written for Andor SOLIS communicated with one written to communicate with the ASI motorized state, run from the shell. These scripts were modified versions of scripts written by Christopher Dedek while he worked in the Murugkar lab in the summer of 2016. The data acquisition from the 9 spots was thus automated. Brightfield images of the first point and the ending point, corresponding to the top left and bottom right of the grid, were recorded to ensure that the grid always remained within the bounds of the targeted nucleus or cytoplasm region.

\subsection{Data preprocessing}

After acquiring the 9 spectra from the $3 \times 3$ grid, an average was calculated. This average spectrum was then baseline subtracted and smoothed, and the final result vector normalized to ensure that all spectra had the same scale. 


\subsubsection{Background subtraction}

The algorithm used to subtract the baseline from the spectrum was a Spectra Method for Iterative Removal of Fluorescence (SMIRF) by Beier et al. ${ }^{60}$ SMIRF iteratively fits a polynomial plus a scaled quartz spectrum to the previous estimate of background, and then takes min(fit,old_bg_est) as the new background estimate. This is repeated until the difference between subsequent background estimates is below a certain tolerance. Least-squares is used to fit the polynomial plus quartz spectrum to each subsequent background estimate. A general outline of the algorithm is given in Algorithm 1. All arithmetic shown in the SMIRF algorithm are performed element-

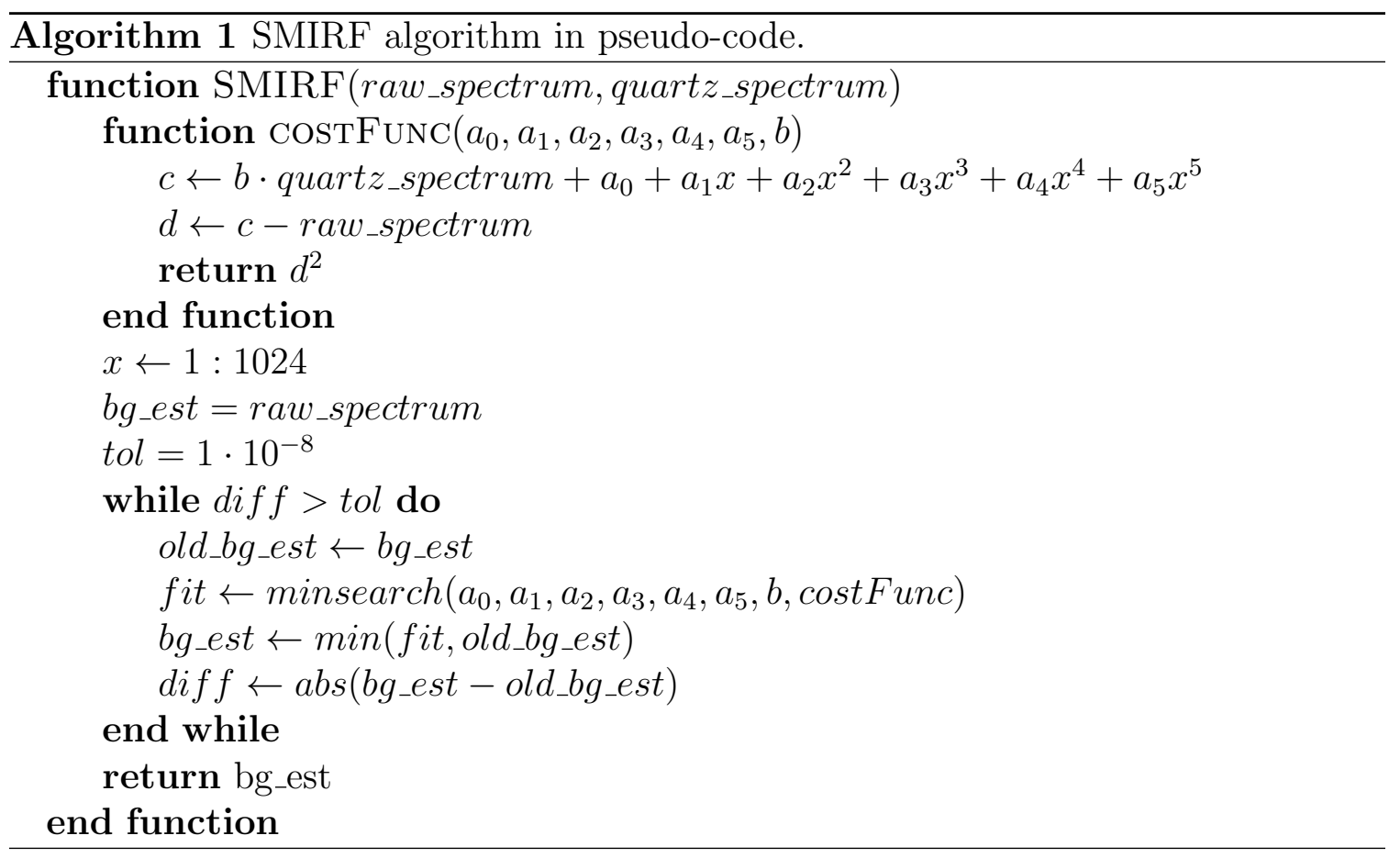

wise on vectors $x$, raw_spectrum, quartz_spectrum, $c$ and $d$. The result of SMIRF applied to an HLE cell spectrum is shown in Figure 4.3. The plot on the left shows the components used in SMIRF, the raw spectrum, the quartz spectrum, the polynomial, and the polynomial plus scaled quartz spectrum fitted to the raw, and on the right the background subtracted spectrum. 

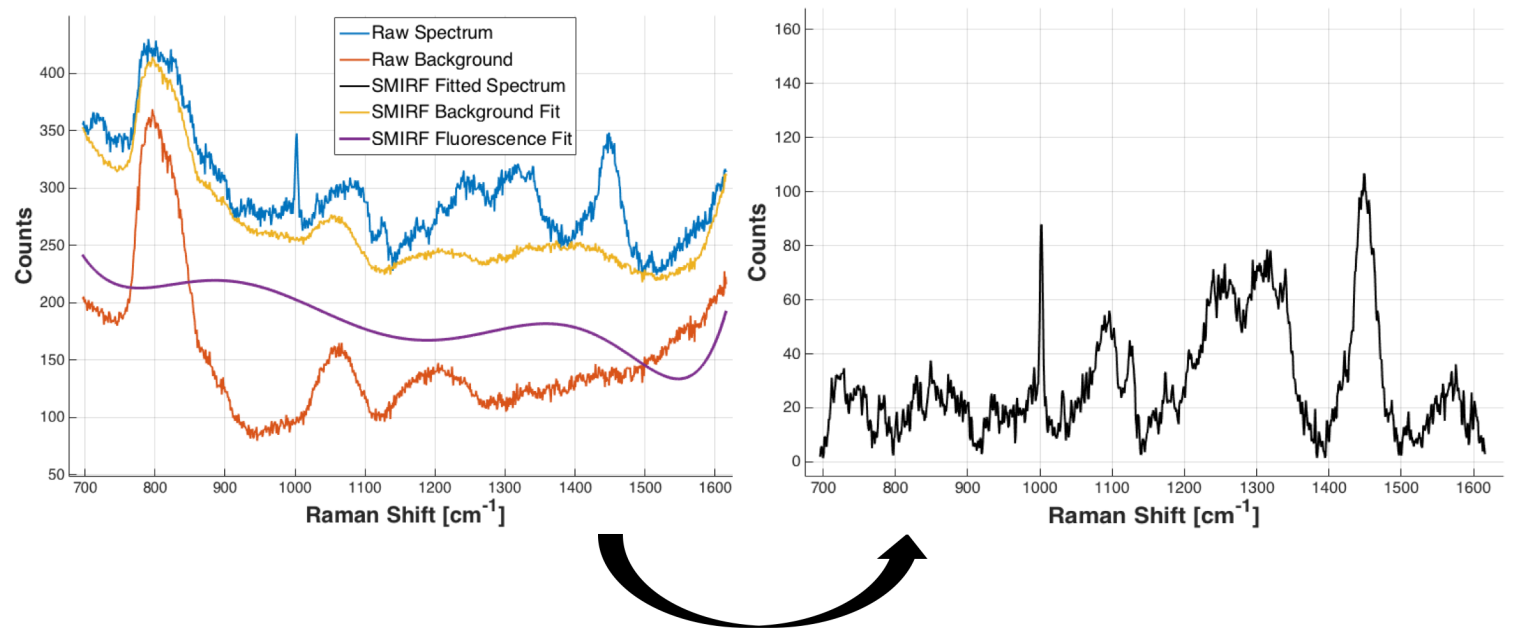

Figure 4.3: Example of SMIRF done to a control HLE spectrum. Raw spectrum, quartz spectrum, polynomial, estimated background, and background subtract spectrum are shown.

\subsubsection{Spectral smoothing}

Smoothing of baseline subtracted spectra was done using a Savitzky-Golay filter. This algorithm is popular in areas of chemical spectral analysis and is useful for reducing noise, and thus increasing $\mathrm{S} / \mathrm{N}$. It takes parameters of window size and polynomial degree, the latter of which is chosen to be of low-degree (e.g. quadratic) in order to produce smoothing. Data points are assumed to be equally spaced, meaning the actual $\mathrm{x}$-axis values are ignored. The algorithm works as follows: for each data point, a polynomial of a chosen order is fit through all data points within a window centred on the given data point using least-square fitting. ${ }^{61}$ The chosen window size must therefore be an odd number to maintain symmetry around each point. The y-value at a given point on the $\mathrm{x}$-axis is then taken as the value of the polynomial at that point which was fitted through the data points in the window centred on that point. ${ }^{62}$ This is done for each wavenumber in the spectrum, and creates a smoother spectrum, with the level of smoothing dependent on both window size and polynomial order. In Raman spectroscopy, for spectrometers with approximately $1 \mathrm{~cm}^{-1}$ bin widths, such 
as the one used in the CBRG, a window size of 15 and polynomial order of 2 is typically used, and that is what was used for this study.

\subsubsection{Vector Normalization}

Vector normalization ensures that, when treated as a vector, each spectrum is a unit vector, and thus differences between them are essentially directional differences in a 1024 dimensional vector space. This is desired as relative differences between peaks correspond to molecular changes in cells, while overall spectral magnitude could be due to many factors, such as cell thickness. Since vector normalization of Raman spectra is the same as normalizing a 1024-dimensional vector in a 1024-dimensional Euclidean space, it is done simply by dividing each spectrum by its Euclidean norm,

$$
\overline{\mathbf{x}}=\frac{\mathbf{x}}{\|\mathbf{x}\|}=\frac{\mathbf{x}}{\sqrt{x_{1}^{2}+x_{2}^{2}+\ldots+x_{1024}^{2}}}
$$

where each $x_{1}, x_{2}, \ldots, x_{1024}$ is the intensity of a wavenumber in the spectrum being normalized. While this is how spectra were normalized in this study, another common method is area normalization, in which $\|\mathbf{x}\|$ is replaced with $\sum_{i=1}^{1024} x_{i}$.

\subsection{Data analysis}

The collected data were analyzed in three primary ways: visually by plotting mean spectra for each dose with $2 \sigma$ confidence intervals around them, statistically by using Welch's $t$-test to determine whether any individual peaks differ significantly to a $5 \%$ signficance level and using multivariate classification techniques to show whether a spectrum from a given dose is distinguishable from control as a whole, and para-

metrically by curve fitting, to demonstrate agreement with the model of low-dose 
hypersensitivity. The linear discriminants found through classification were also used to identify possible changes in cellular biochemistry induced by IR. The primary aim of these techniques were to open up avenues of further investigation, as this was primarily an investigative study.

\subsubsection{Welch's t-test}

Welch's $t$-test is a more robust, generalized form of Student's two-sample $t$-test, ${ }^{63}$ in that it does not assume the two distributions have the same variance or sample size. Like Student's two-sample $t$-test, it is a statistical test that can be used to determine the probability that two sets of sample data come from distributions with the same mean value. This is the hypothesis it tests when a two-tailed test is performed. A one tailed test is used when one wishes to determine if one set of sample data comes from a distribution with a mean greater or lesser than another set of sample data, with which tail is used determining which inequality is being tested. When they do, it becomes equivalent to Student's two-sample $t$-test.

The test statistic for Welch's $t$-test is given by

$$
t=\frac{\bar{X}_{1}-\bar{X}_{2}}{\sqrt{\frac{s_{1}^{2}}{N_{1}}+\frac{s_{2}^{2}}{N_{2}}}},
$$

where $\bar{X}_{1}$ and $\bar{X}_{2}$ are the sample means for the two datasets, $s_{1}^{2}$ and $s_{2}^{2}$ are their sample variances, and $N_{1}$ and $N_{2}$ their sample sizes. It can be seen that when $s_{1}^{2}=s_{2}^{2}$ and $N_{1}=N_{2}=N$, Equation 4.3 reduces to

$$
t=\frac{\bar{X}_{1}-\bar{X}_{2}}{s_{p} \sqrt{\frac{2}{N}}}
$$

which is Student's two-sample $t$-test. Note that when $s_{1}^{2}=s_{2}^{2}$, the pooled sample 
standard deviation, $s_{p}$, is equal to $\sqrt{\left(s_{1}^{2}+s_{2}^{2}\right) / 2}$. The test statistic, $t$, for Welch's two-sample $t$-test (it can generalize to comparing more than two sample distributions) will have an approximate Student's $t$-distribution with degrees of freedom given by

$$
f=\frac{\left(\frac{s_{1}^{2}}{N_{1}}+\frac{s_{2}^{2}}{N_{2}}\right)^{2}}{\frac{\left(s_{1}^{2} / N_{1}\right)^{2}}{N_{1}-1}+\frac{\left(s_{2}^{2} / N_{2}\right)^{2}}{N_{2}-1}}{ }^{63}
$$

Getting the value $t$ itself and testing its significance on both tails of the corresponding t-distribution was automatically handled by MATLAB's ttest2 $(\mathrm{x} 1, \mathrm{x} 2)$ function, which defaults to a two-tailed significance test for a significance level of $p<0.05$. This was tested on each dose-control pair for each Raman shift number.

\subsubsection{PCA-LDA}

PCA-LDA was used to determine whether the spectra from each dose set can be discriminated from the control set. This was done using the four batches for which a viable set of spectra were attained for each dose. Some batches could not be used due to fluorescence in some of the quartz substrates. Leave-one-out cross-validation (LOOCV) was used to estimate the sensitivity, specificity, and accuracy of each discrimination. Using four batches, in which there are 17 nucleus and 16 cytoplasm spectra per dose, gave a whole dataset of 68 nucleus and 64 cytoplasm spectra for each dose. The script to do this and perform the LOOCV was written in-house using the $\mathrm{R}$ programming language, with the PCA and LDA algorithms performed by functions pricomp and lda in the stats and MASS packages respectively. The preferred method for computing the PCs in pricomp is via singular-value decomposition of the centered data matrix (see Appendix A.4), and so mean centring the data was a necessary first step.

A common method for deciding how many PCs to use is typically to use enough 
to account for some percent of the variance in a given dataset. ${ }^{56,64}$ If PCA is being used only for dimensionality reduction in order to reduce the chance of overfitting a training set when used in conjunction with a supervised classifier, in this case LDA, then this assumes that the features most useful for classification between classes are those with the highest variance. It also assumes that the chosen percent of variance to account for will be explainable by the first few PCs. While it is true that features that account for very little variance tend to correspond to noise, this correspondence is gradual. So while it makes sense to start with PCs that account for most variance, it also makes sense to choose the cutoff such that LOOCV accuracy reaches some optimum point. Plotting LOOCV accuracy as a function of number of PCs used showed that the accuracy tended to plateau at a certain point. Since using more PCs after this point has little, or even negative effects on accuracy, it was decided that those higher PCs don't correspond to dose related changes, and lead to overfitting the training set. It should be noted that PCA is sensitive to any source of variability in the data, which could include variability between batches. It is therefore important to ensure that each batch has the same set of doses, otherwise a PC corresponding to batch variance could also correspond to dose variance for some doses, which would lead to misleading classification results when applying LDA.

An example of such a plot is given in Figure 4.4. The optimum point was chosen as the shoulder of the plot, which was visible in each dose-control pair. The exact point was chosen manually.

The LD loadings from the chosen LDA (i.e. using the optimum number of PCs) were then extracted. Those Raman shifts with the highest loadings for each dosecontrol comparison were then matched to known Raman peaks of various biomolecules. This was used to determine which biomolecules were changing in structure or concentration due to the radiation exposure. Unfortunately this processes is currently done only by matching Raman shift numbers to a known source, and thus does not 


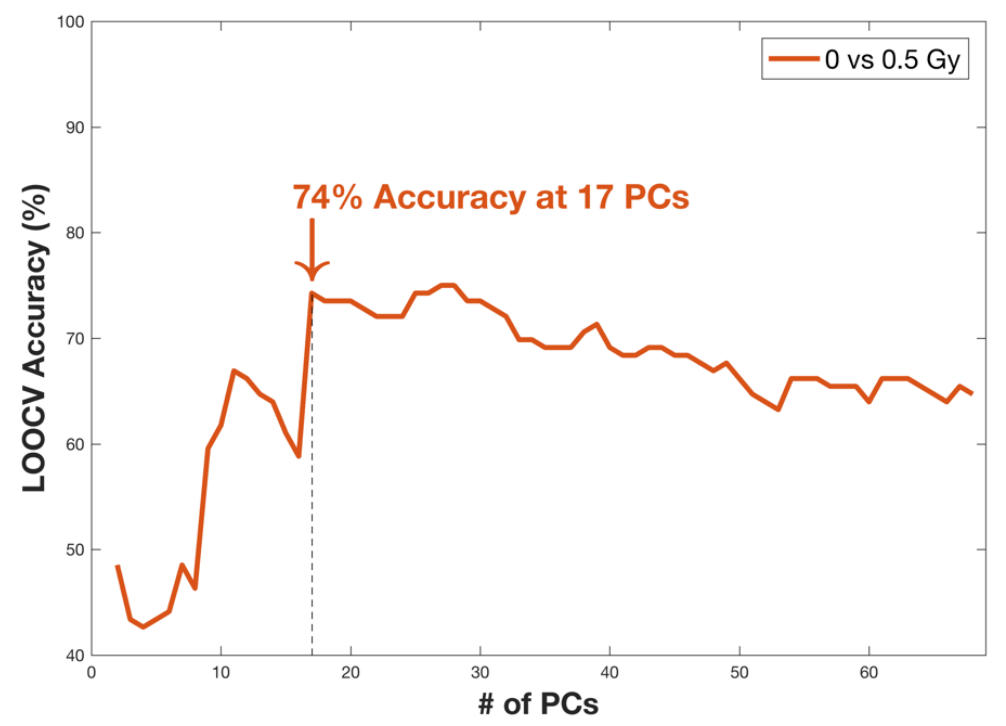

Figure 4.4: Plot of Leave-one-out cross-validation accuracy vs number of PCs used in the discrimination.

provide a statistical estimate for the probability that a peak or set of peaks belongs to some molecule or another. It is still useful for exploratory studies, however, as it allows one to hypothesize on the possible biological changes at play, and plan future paths of investigation.

\subsubsection{Curve fitting}

The induced repair model of cell survival, given by Equation 2.8, was fit to the data from a clonogenic assay performed at Health Canada, as well as to the band area absolute difference ratio of certain peaks. The absolute difference ratio is calculated via

$$
Y=1-\frac{\left|A_{0}-A_{D}\right|}{A_{0}}
$$

where $A_{0}$ is the area under a given peak for the control set, and $A_{D}$ is the area under the peak for a given dose. The mean and standard deviation of these values were then found, and Equation 2.8 used to fit the data. This method of relating band area 
difference rations (Eq. 4.6) to induce repair survival curves was first demonstrated by Meade et al. on human keratinocytes. ${ }^{25}$. The code used to fit the HLE data was written in-house using MATLAB v. 2016b (MathWorks Inc., Natick, MA, USA). 


\section{Chapter 5}

\section{Results \& Discussion}

\subsection{Acquisition of Raman Spectra from Nucleus and Cytoplasm}

\subsubsection{Brightfield images of HLE cells}

Brightfield images of each cell were made before and after acquiring spectra from a given location. The brightfield images thus showed the starting point (top-left most point in a grid) and ending point (bottom-right most point on grid) of a measurement. Figure 5.1 a) shows a brightfield of HLE cells with arrows pointing out nuclei and cyctoplasm. Three nuclei are also circled to indicate their approximate size. Overlaid on top of one of the nuclei is a $3 \times 3$ point grid with appropriate dimensions. Figure $5.1 \mathrm{~b})$ shows the same image without any overlaid annotation, and with increased sharpness and contrast to make the nucleus boundaries more visible.

Each spectrum was acquired with the laser spot focused $5 \mu \mathrm{m}$ above the quartz substrate, as this was found to be the optimal height at which to acquire spectra 


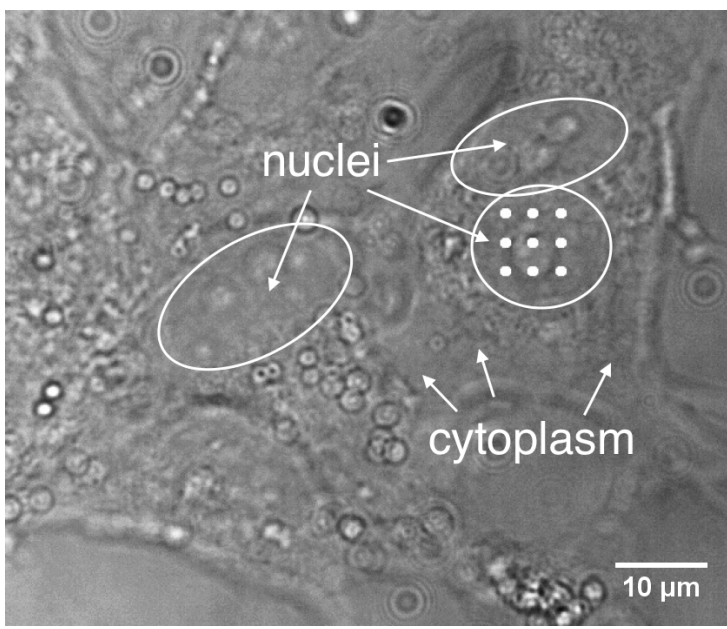

(a)

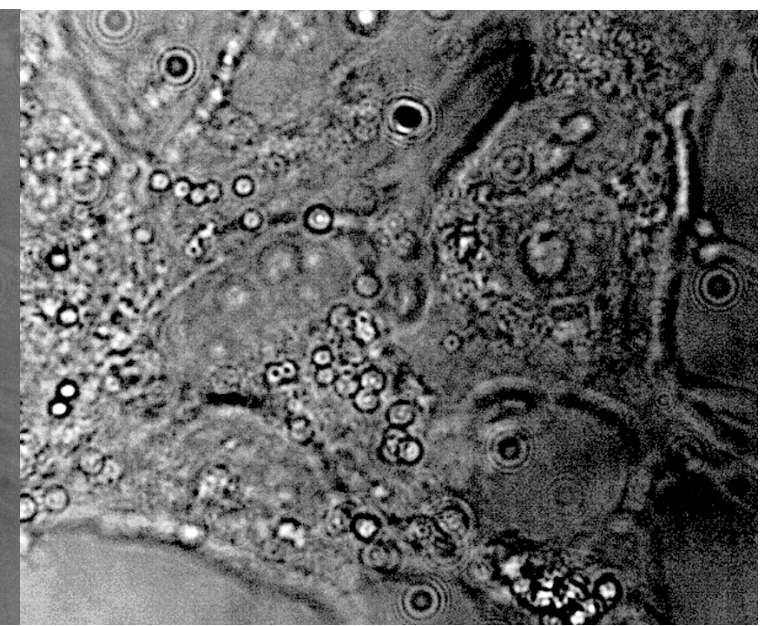

(b)

Figure 5.1: a) Brightfield image of HLE cells with 3x3 point grid shown over a nucleus. Grid dimensions are $6 \mu \mathrm{m} \times 6 \mu \mathrm{m}$. b) Same image without overlaid annotation and increased contrast and sharpness to show nucleus boundaries. The smaller circular objects within the nucleus are nucleoli.

from HLE cells adhered to quartz slips. This was determined by acquiring spectra at different heights above the quartz substrate for several HLE cells, beginning with the laser in focus at the substrate, and increasing the height (z-axis) by $1 \mu \mathrm{m}$ increments. The spectral intensity was found by taking the norm (Euclidian distance) of each spectrum as a whole, after background subtraction. Each spectrum was visually inspected as well, and it was found that the Raman peak intensities for the HLE cells were maximized at between $3 \mu \mathrm{m}$ and $6 \mu \mathrm{m}$. A plot of spectral norm vs z-depth is given in Figure 5.2. A height of $5 \mu \mathrm{m}$ was found to be an acceptable compromise between have the focal volume intersect the target cell as much as possible and intersect the quartz substrate as little as possible.

\subsubsection{Nucleus spectra}

Using the average spectrum from a grid of 9 point-spectra for a single measurement was necessary due to the heterogeneity within each cell at the micron scale. This is 


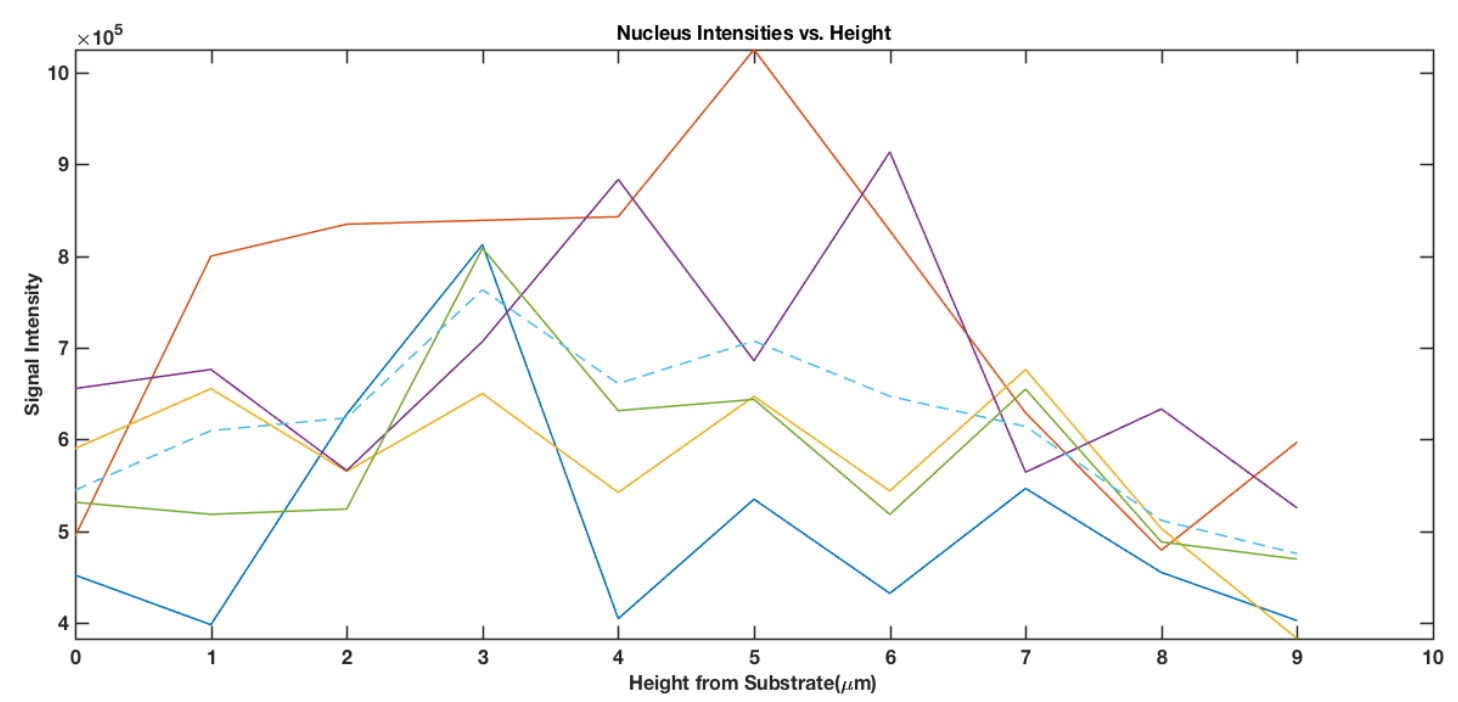

Figure 5.2: Plot of spectral intensity vs distance above quartz surface for several cells. Laser was centred on HLE nucleus for each cell. Each solid line corresponds to a single cell. The dashed line is the average spectral intensity.

demonstrated by Figure 5.3, which shows the plots of Raman spectra for each point of a 3x3 grid from a non-irradiated HLE nucleus. The final average of all 9 mean spectra is given in Figure 5.4. A $2 \sigma$ confidence interval, where $\sigma$ is the standard deviation, is included.

The mean nucleus spectrum of all 68 measurements from the control (nonirradiated) set is given in Figure 5.5 below. Note that this is after background removal, so the large fluorescent line shape corresponding to quartz around $800 \mathrm{~cm}^{-1}$ in the previous plots is removed. The main Raman peaks correspond to lipids, various proteins, DNA base pairs, and metabolites. Individual peaks often correspond to multiple biomolecules, and partially overlapping peaks from different biomolecules create large bands of signal, with small peaks along them corresponding to the individual peaks that make up the band. Sometimes these peaks are difficult to discern, and so one can only list possible contributing molecules without attempting more sophisticated methods. Table 5.1 lists molecular assignments for prominent peaks and bands in the mean nucleus control spectrum. 

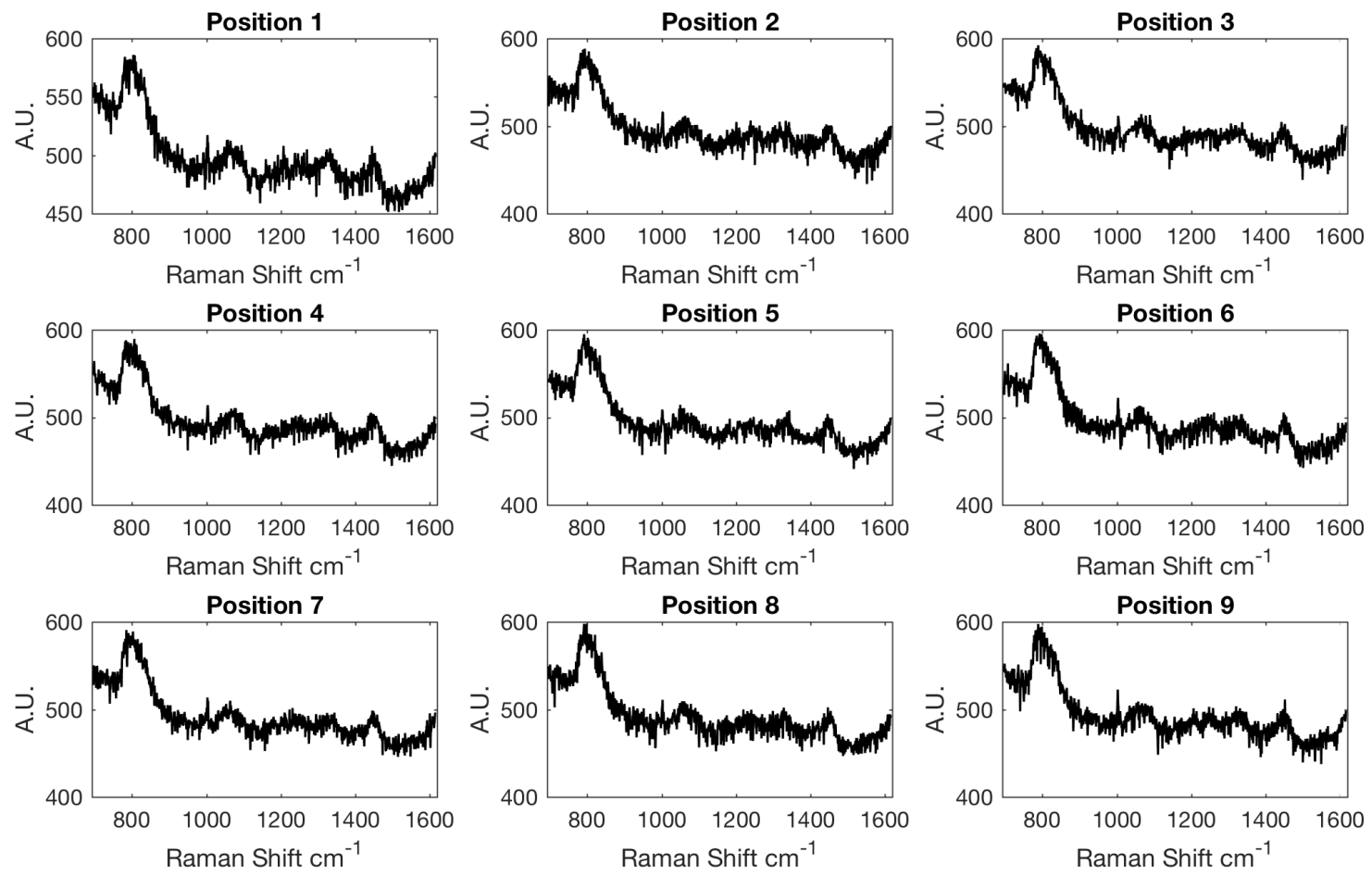

Figure 5.3: Individual nucleus point spectra from a 3x3 point grid covering a $6 \times 6 \mu \mathrm{m}$ area. 


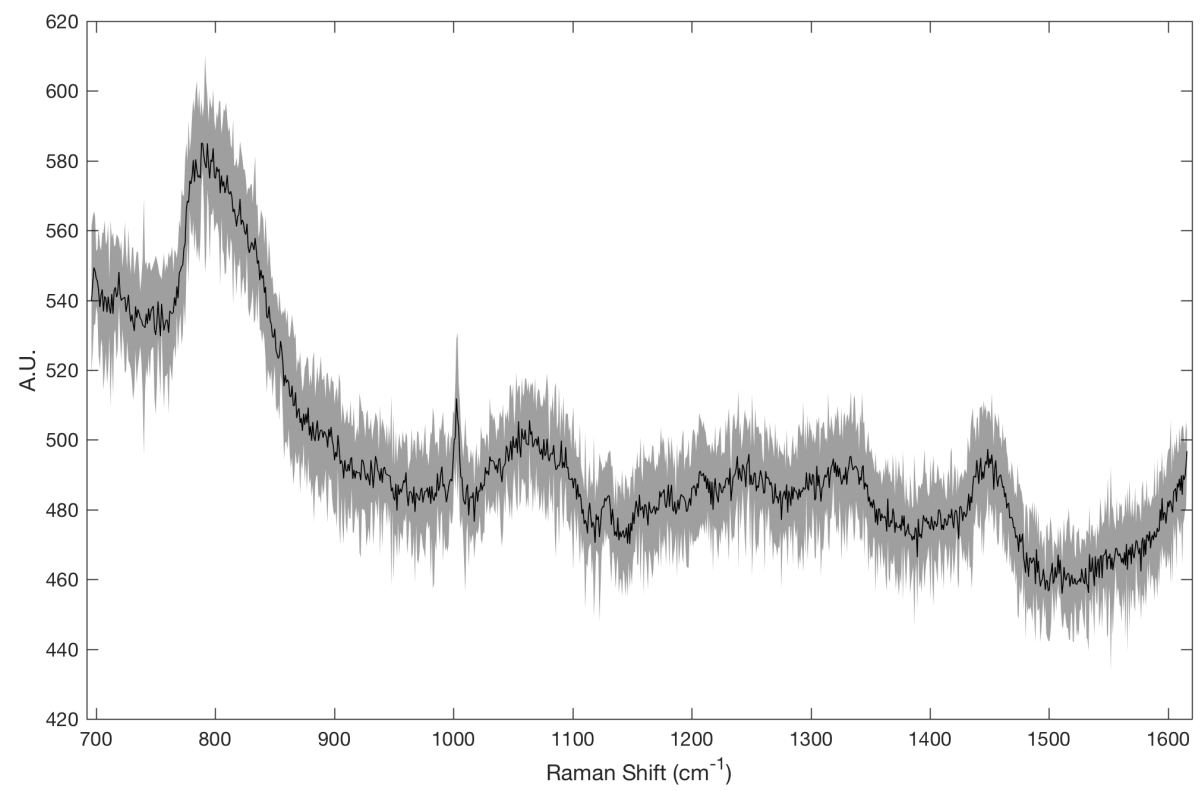

Figure 5.4: Mean of 9 point spectra for an individual nucleus covering a $6 \times 6 \mu \mathrm{m}$ area.

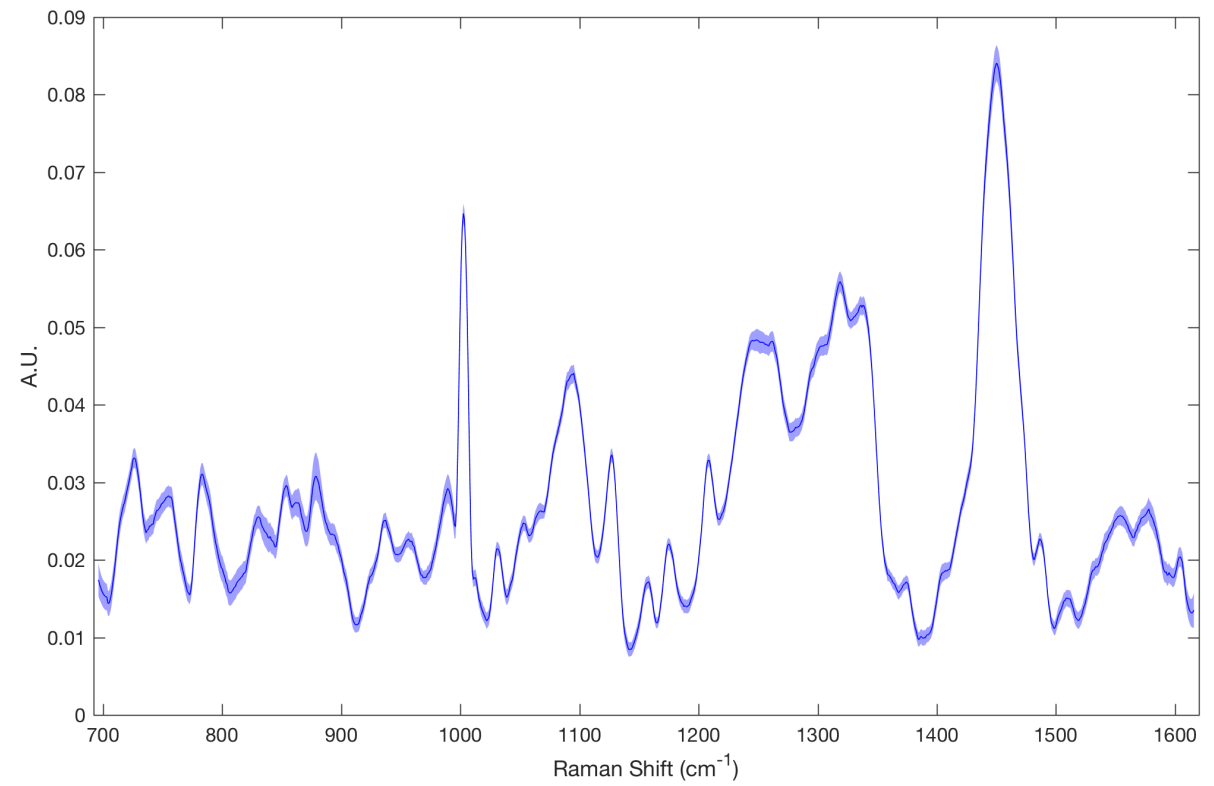

Figure 5.5: Mean Raman spectrum for nucleus using all 68 spectra from the control set. 
Table 5.1: Peak assignments for prominent peaks of mean control nucleus spectrum. Abbreviations: (A) adenine, (U) uracil, (T) thymine, (C) cytosine, (G) guanine, (p) protein, (l) lipid, (s) saccharide, (m) metabolite, (d) DNA or RNA, (str) stretch, (bk) backbone, and (br) breathing. References are given by bracketed number.

\begin{tabular}{|c|c|}
\hline $\begin{array}{l}\text { Peak } \\
\left(\mathrm{cm}^{-1}\right)\end{array}$ & Molecular Assignment(s) \\
\hline 717 & Choline $^{65}$ \\
\hline 748 & T ring br $(d)^{66}$ \\
\hline 782 & $\mathrm{U}, \mathrm{C}$, T ring br; O-P-O str bk (d) ${ }^{65,66}$ \\
\hline 828 & O-P-O asym str $\mathrm{T}$ ring br (d) ${ }^{65,66}$ \\
\hline 852 & Proline, ${ }^{66}$ Tyrosine ring br $(\mathrm{p})^{65}$ \\
\hline 865 & Tryptophan $(\mathrm{p})^{67}$ \\
\hline 878 & Acyl $\mathrm{C}_{2}-\mathrm{C}_{1} \operatorname{str}(\mathrm{l})^{65}$ \\
\hline 937 & C-C sym str bk; $\alpha$-helix (p) ${ }^{65}$ \\
\hline 989 & Glutathione ${ }^{67}$ \\
\hline 1002 & Phenylalanine ring br ${ }^{68}$ \\
\hline 1032 & C-H Phenylalanine $(\mathrm{p})^{65}$ \\
\hline 1055 & Glycerol (l); D-glucose (s) ${ }^{67}$ \\
\hline 1094 & $\mathrm{PO}_{2^{-}}$Sym str of DNA/RNA (d) ${ }^{65}$ \\
\hline 1127 & $\begin{array}{l}\text { Glucosamine }(\mathrm{s}) ;{ }^{65,67} \mathrm{C}-\mathrm{N} \text { str }(\mathrm{p}) ;{ }^{66} \\
\text { lauric acid }(\mathrm{l})^{67}\end{array}$ \\
\hline 1175 & Tyrosine, Phe $(\mathrm{p})^{65}$ \\
\hline 1247 & Amide III beta-sheet $(\mathrm{p})^{65}$ \\
\hline 1260 & Amide III $\alpha$-helix $(\mathrm{p})^{66}$ \\
\hline 1303 & $\mathrm{CH}_{3}, \mathrm{CH}_{2}$ twisting ${ }^{66}$ \\
\hline 1320 & DNA base $(d) ; C H$ def $(p)^{65}$ \\
\hline 1335 & $\mathrm{~A}, \mathrm{G}(\mathrm{d}) ; \mathrm{C}-\mathrm{H} \operatorname{def}(\mathrm{p}), \mathrm{CH}_{2}$ twist $^{69}$ \\
\hline 1450 & $\mathrm{CH}_{2}$ bending and $\mathrm{CH}_{3} \operatorname{def}(\mathrm{l})(\mathrm{p})^{65}$ \\
\hline 1486 & $A, G(d)^{65}$ \\
\hline 1508 & Valine $(p)^{67}$ \\
\hline 1550 & $G(d)^{67}$ \\
\hline 1577 & $\mathrm{~A}, \mathrm{G}^{65}$ \\
\hline 1605 & C-C Pheylalanine, Tyrosine ${ }^{65}$ \\
\hline
\end{tabular}




\subsubsection{Cytoplasm Spectra}

The cytoplasm spectra were taken over a much smaller area, with adjacent sampled points being just $1 \mu \mathrm{m}$ apart. A plot of 9 point spectra from $2 \times 2 \mu \mathrm{m}$ grid is given in Figure 5.6, followed by the average of those 9 spectra in Figure 5.7.
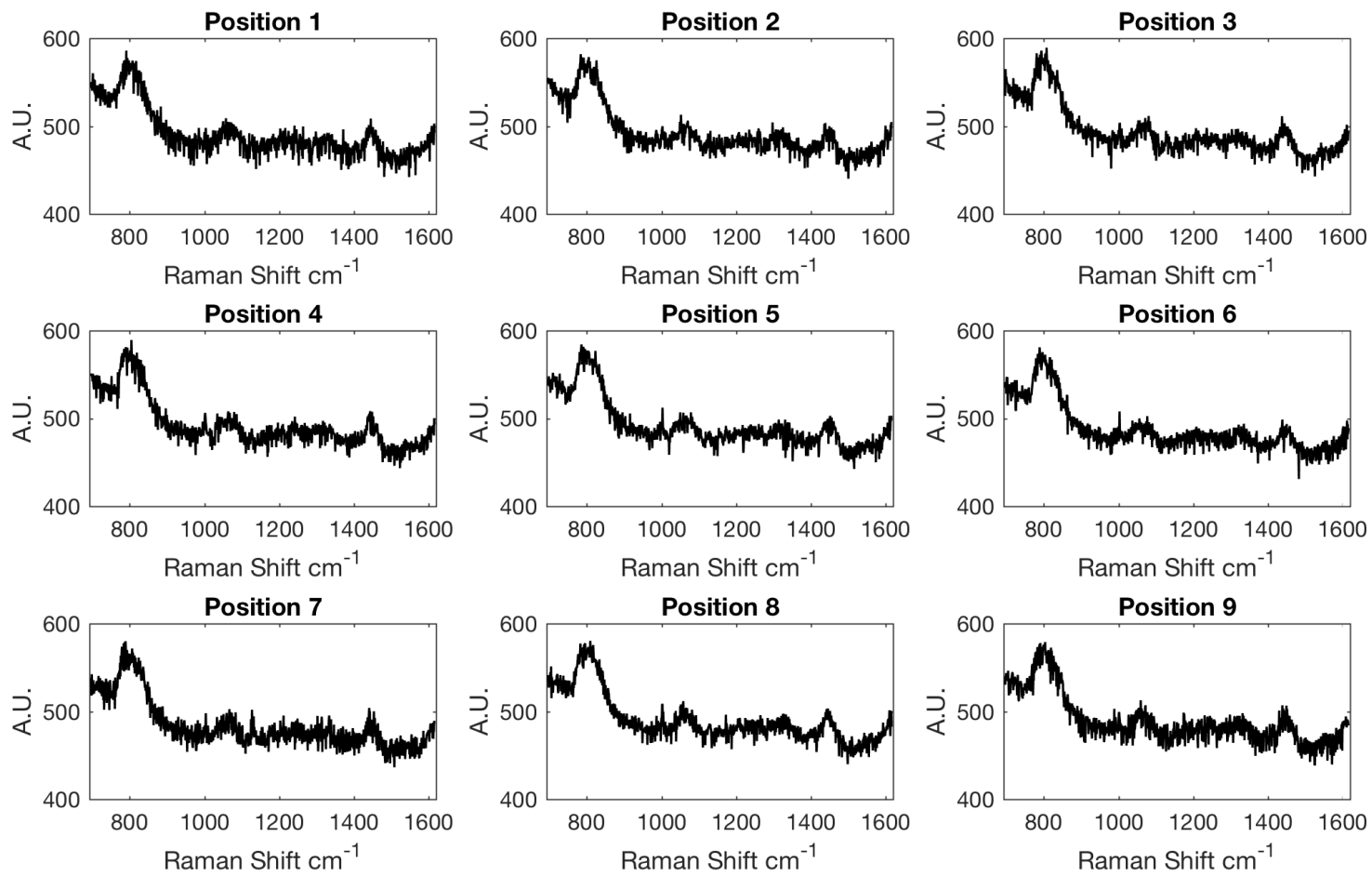

Figure 5.6: Individual point spectra over a $2 \times 2 \mu \mathrm{m}$ area of cytoplasm.

The mean cytoplasm spectrum of all 64 measurements from the control (nonirradiated) set is given in Figure 5.8. Again, this mean spectrum is from spectra processed with background subtraction, and so does not contain quartz fluorescence around $800 \mathrm{~cm}^{-1}$ which is visible in the raw spectra. Table 5.2 lists molecular assignments for prominent peaks and bands in the mean cytoplasm control spectrum. 


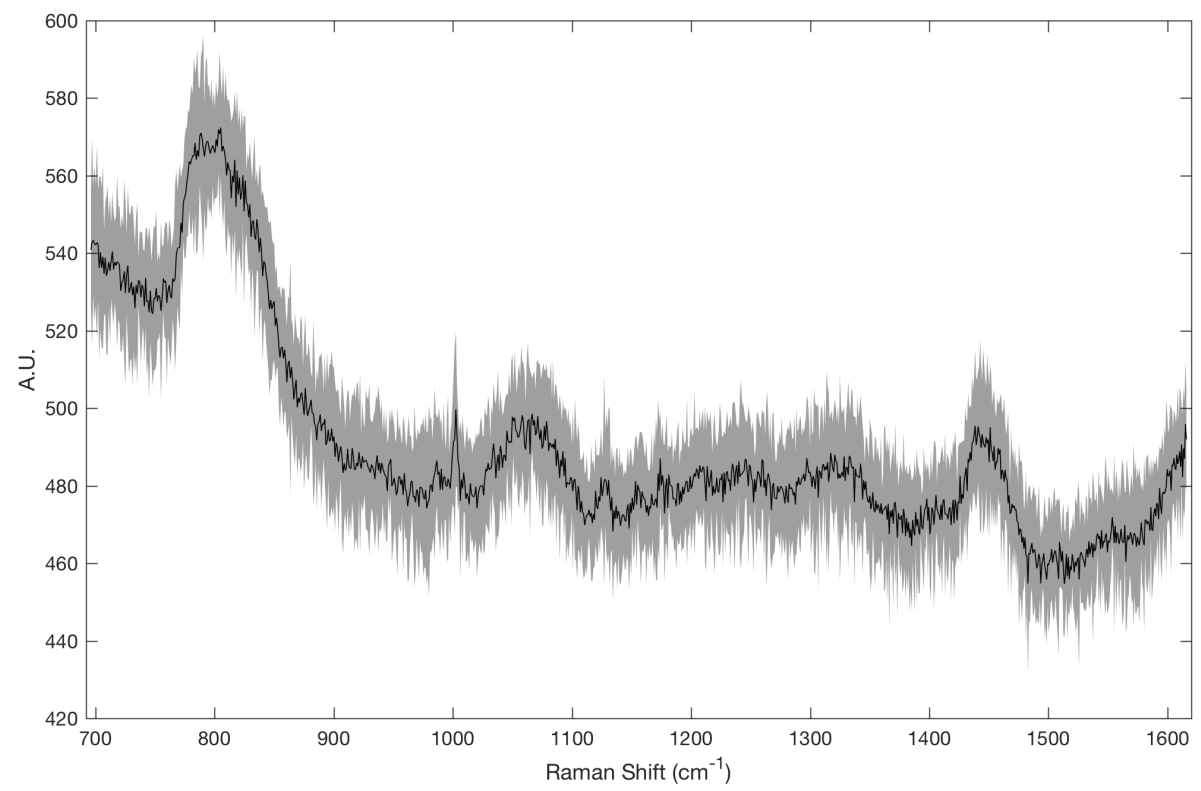

Figure 5.7: Mean of 9 point spectra for an individual section of cytoplasm covering a $2 \times 2 \mu \mathrm{m}$ area.

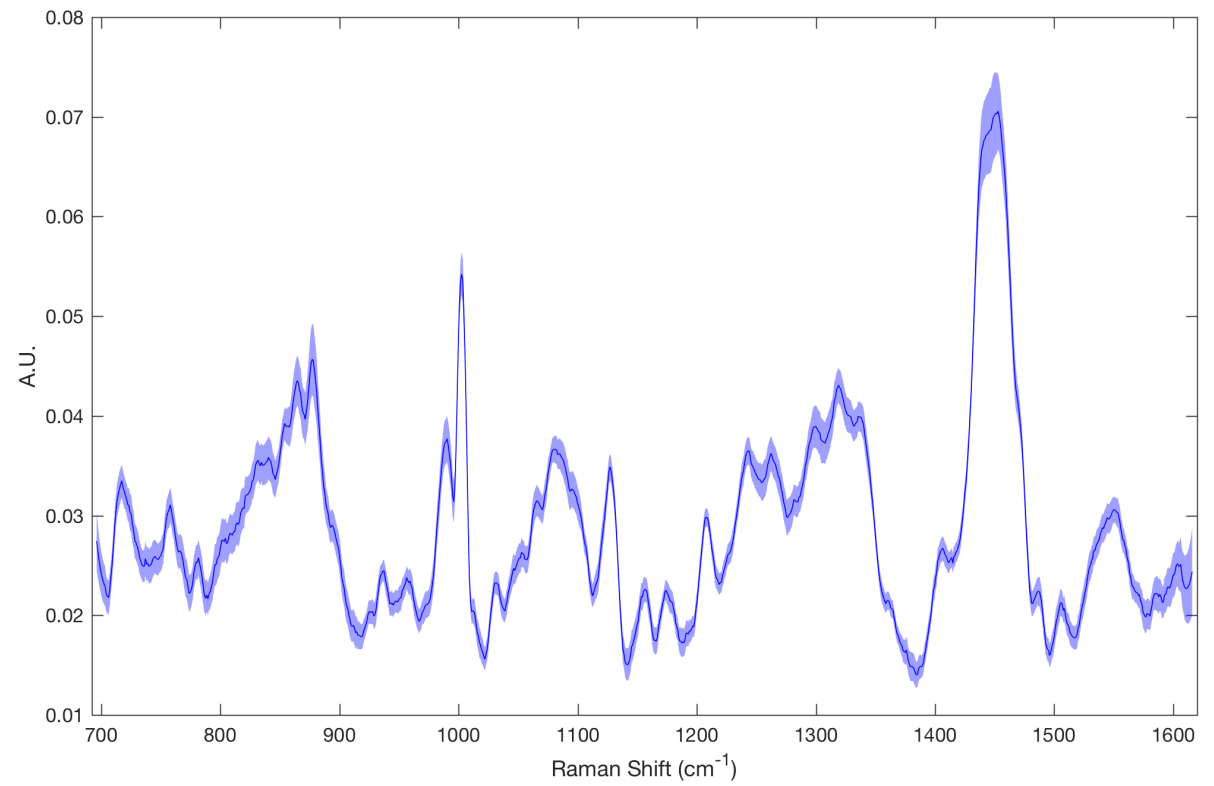

Figure 5.8: Mean Raman spectrum for cytoplasm using all 64 spectra from the control set. 
Table 5.2: Peak assignments for prominent peaks of mean control cytoplasm spectrum. Abbreviations: (A) adenine, (U) uracil, (T) thymine, (C) cytosine, ( $\mathrm{G}$ ) guanine, (p) protein, (l) lipid, (s) saccharide, (m) metabolite, (d) DNA or RNA, (str) stretch, (bk) backbone, and (br) breathing. References are given by bracketed number.

\begin{tabular}{|c|c|}
\hline $\begin{array}{l}\text { Peak } \\
\left(\mathrm{cm}^{-1}\right)\end{array}$ & Molecular Assignment(s) \\
\hline 747 & Phenylalanine $(\mathrm{p})^{67}$ \\
\hline 755 & Tryptophan $(\mathrm{p})^{67}$ \\
\hline 804 & $\mathrm{~T}(\mathrm{~d}) ; \operatorname{tryptophan}(\mathrm{p})^{67}$ \\
\hline 811 & Glutathione; O-P-O str RNA (d) ${ }^{65}$ \\
\hline 832 & Phenylalanine $(\mathrm{p})^{67}$ \\
\hline 841 & Trilinolenin (l); glucose, dextrose $(\mathrm{s})^{67}$ \\
\hline 865 & Tryptophan $(\mathrm{p})^{67}$ \\
\hline 877 & Proline (p); ${ }^{67}$ Acyl $\mathrm{C}_{2}-\mathrm{C}_{1} \operatorname{str}(\mathrm{l})^{65}$ \\
\hline 896 & Cellulose $(\mathrm{s})^{67}$ \\
\hline 988 & Glutathione ${ }^{67}$ \\
\hline 1002 & Phenylalanine ring br ${ }^{68}$ \\
\hline 1032 & C-H Phenylalanine; ${ }^{65} \mathrm{NAD}^{70}$ \\
\hline 1080 & $\mathrm{C}-\mathrm{N} \operatorname{str}(\mathrm{p})$; chain $\mathrm{C}-\mathrm{C}$ str $(\mathrm{l})^{67}$ \\
\hline 1084 & $\mathrm{NAD}, \mathrm{NADH}^{70}$ \\
\hline 1127 & $\begin{array}{l}\text { Glucosamine }{ }^{67} \mathrm{C}-\mathrm{N} \text { str }(\mathrm{p}){ }^{66} \text { lauric acid }(\mathrm{l}) ;^{67} \\
\mathrm{C}-\mathrm{N}(\mathrm{p}) \text { and chain C-C str }(\mathrm{l}){ }^{65}\end{array}$ \\
\hline 1158 & $\mathrm{C}-\mathrm{C}, \mathrm{C}-\mathrm{N} \operatorname{str}(\mathrm{p})^{66}$ \\
\hline 1175 & Tyrosine, Phenylalanine $(\mathrm{p})^{65}$ \\
\hline 1208 & $\mathrm{C}_{-} \mathrm{C}_{6} \mathrm{H}_{6}$ str Phenylalanine, Tryptophan, Tyrosine $(\mathrm{p})^{65}$ \\
\hline 1247 & Amide III $\beta$-sheet $(p)^{65}$ \\
\hline 1262 & Amylose $(\mathrm{s})^{67}$ \\
\hline 1282 & Glutamate $(\mathrm{p})^{67}$ \\
\hline 1298 & Arginine (p); Lauric acid (l) ${ }^{67}$ \\
\hline 1300 & Amide III; ${ }^{66} \mathrm{CH}_{2} \operatorname{def}(\mathrm{l})^{65}$ \\
\hline 1321 & N-Acetyl-D-glucosamine; Valine; ${ }^{67}$ Amide III ${ }^{66}$ \\
\hline 1336 & $\begin{array}{l}\text { Phenylalanine }(\mathrm{p}) ; \text { Histidine }(\mathrm{p}) ; \mathrm{D}-(+) \text {-Galactosamine }(\mathrm{s}) ;^{67} \\
\text { Polynucleotide chain }{ }^{66}\end{array}$ \\
\hline 1407 & Coenzyme A $(\mathrm{m})$; Histidine $(\mathrm{p})^{67}$ \\
\hline 1440 & $\mathrm{CH}_{2} \operatorname{def}(\mathrm{l})^{65,67}$ \\
\hline 1450 & Tryptophan $(\mathrm{p}) ;^{67} \mathrm{CH}_{2}$ bending mode ${ }^{66}$ \\
\hline 1486 & Tryptophan $(\mathrm{p})^{67}$ \\
\hline 1505 & Valine $(\mathrm{p})^{67}$ \\
\hline 1550 & Guanine $^{67}$ \\
\hline 1586 & Phenylalanine $(\mathrm{p})^{66,67}$ \\
\hline 1605 & Glutamate, proline $(\mathrm{p}) ;{ }^{67} \mathrm{C}$, Phenylalanine, tyrosine ${ }^{66}$ \\
\hline
\end{tabular}




\subsection{Differences between mean dose and mean con- trol spectra}

Observable differences were found between the mean Raman spectrum for each dose compared to the control dose (0 Gy). These differences can be seen in Figures 5.9 (nucleus) and 5.11 (cytoplasm), in which the mean of the vector normalized Raman spectra of each dose are plotted on top of the control mean (blue). Each spectrum has a $95 \%$ confidence interval, taken as $2 \sigma_{m}$, where $\sigma_{m}$ is the standard deviation of the mean.

Welch's $t$-test (two-tailed) was used to determine the statistical significance of the difference between the sample means for each Raman shift wavenumber, and many peaks were found to have a p-value of less than $5 \%$, and some less than $1 \%$. The differences are illustrated more prominently in Figures 5.10 (nucleus) and 5.12 (cytoplasm), in which bands showing clearly visible difference between a given mean dose and control spectrum are plotted over a smaller scale. While the differences between means are statistically significant, the dose and control populations do not differ enough at any given band to attain a high discrimination accuracy based on that band alone, making the use of multivariate statistical techniques a necessity for distinguishing between dose and control. 


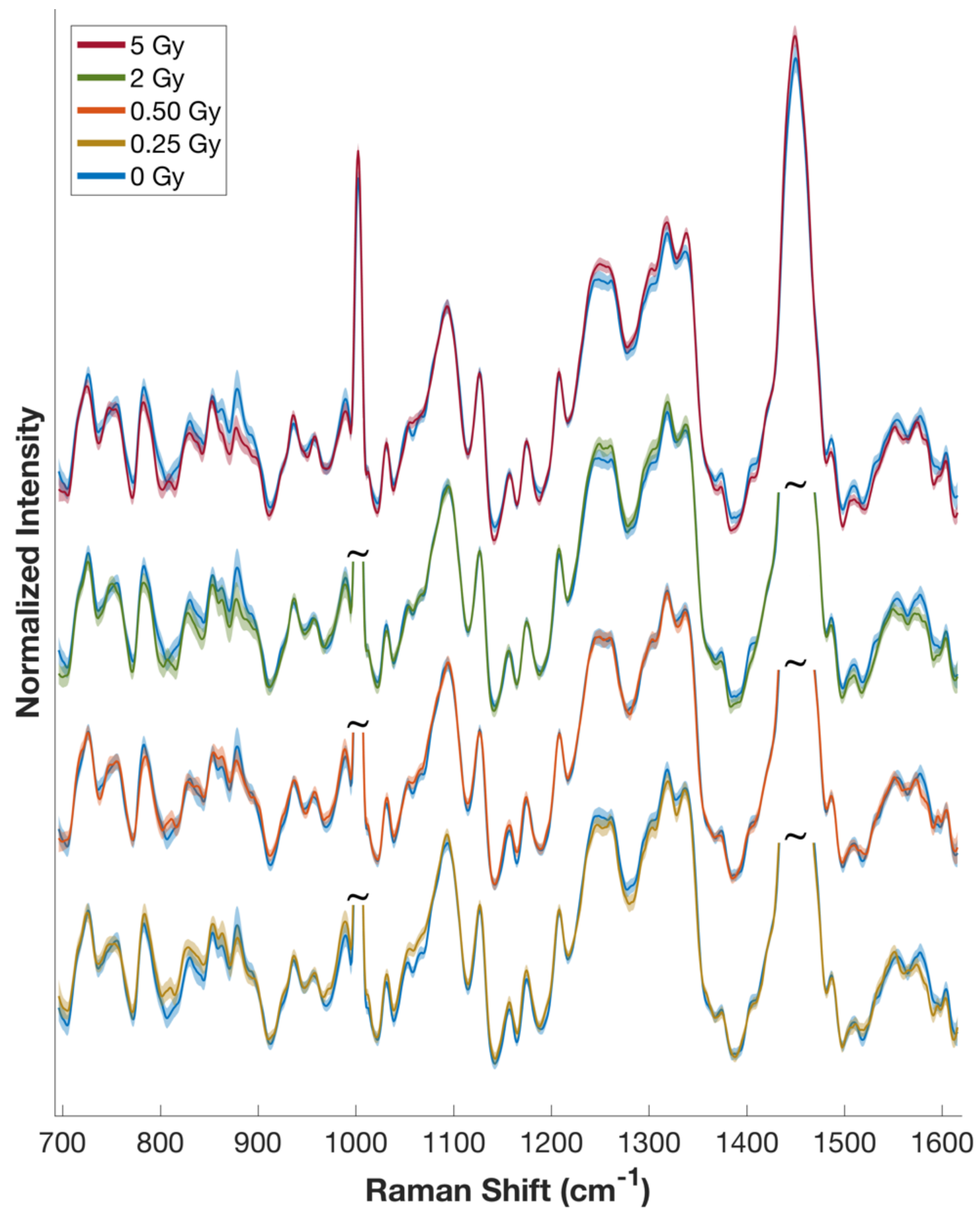

Figure 5.9: Mean nucleus spectra at $0.25,0.5,2$, and 5 Gy, each plotted with 0 Gy spectrum (blue) along with a 95\% confidence interval. Large peaks have been removed for compactness, with cuts marked by . A vertical offset has been added between different dose spectra for improved visual clarity. 


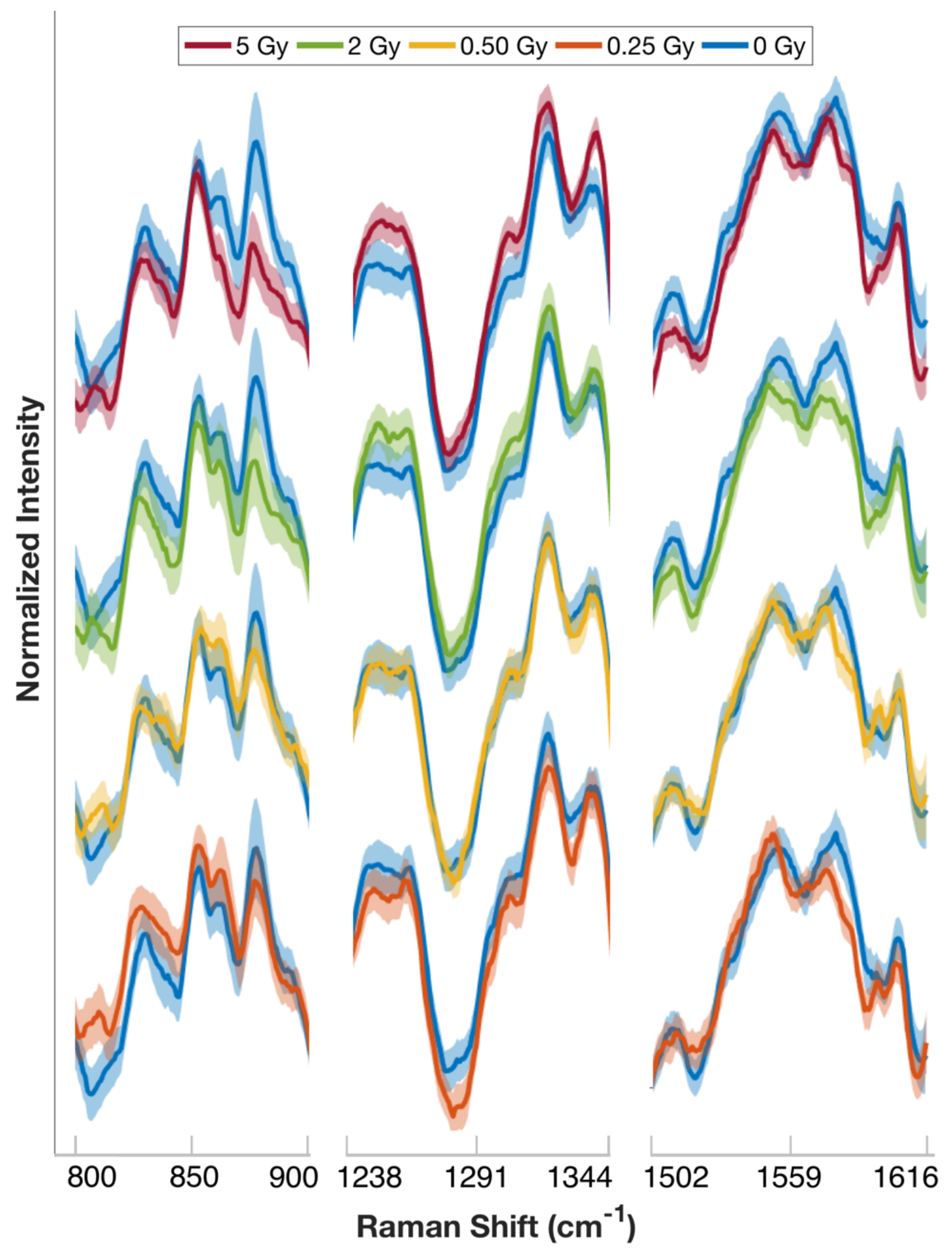

Figure 5.10: Zoomed-in view of some sections of the nucleus spectra in Figure 5.9. Note that these segments are from three spectral bands, and so the x-axis is discontinuous between segments. 


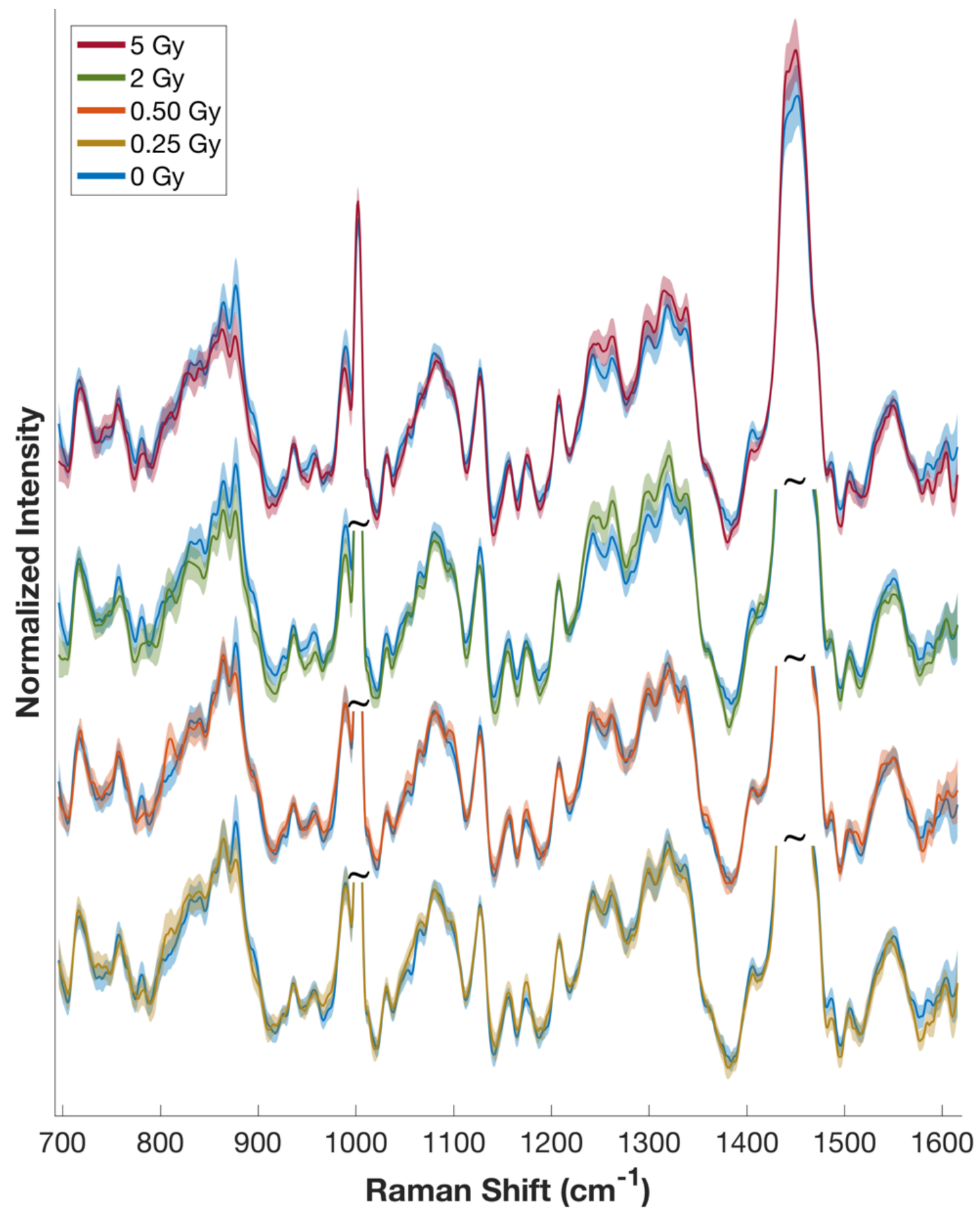

Figure 5.11: Mean cytoplasm spectra at $0.25,0.5,2$, and 5 Gy, each plotted with 0 Gy spectrum (blue) along with a $95 \%$ confidence interval. Large peaks have been removed for compactness, with cuts marked by . A vertical offset has been added between different dose spectra for improved visual clarity. 


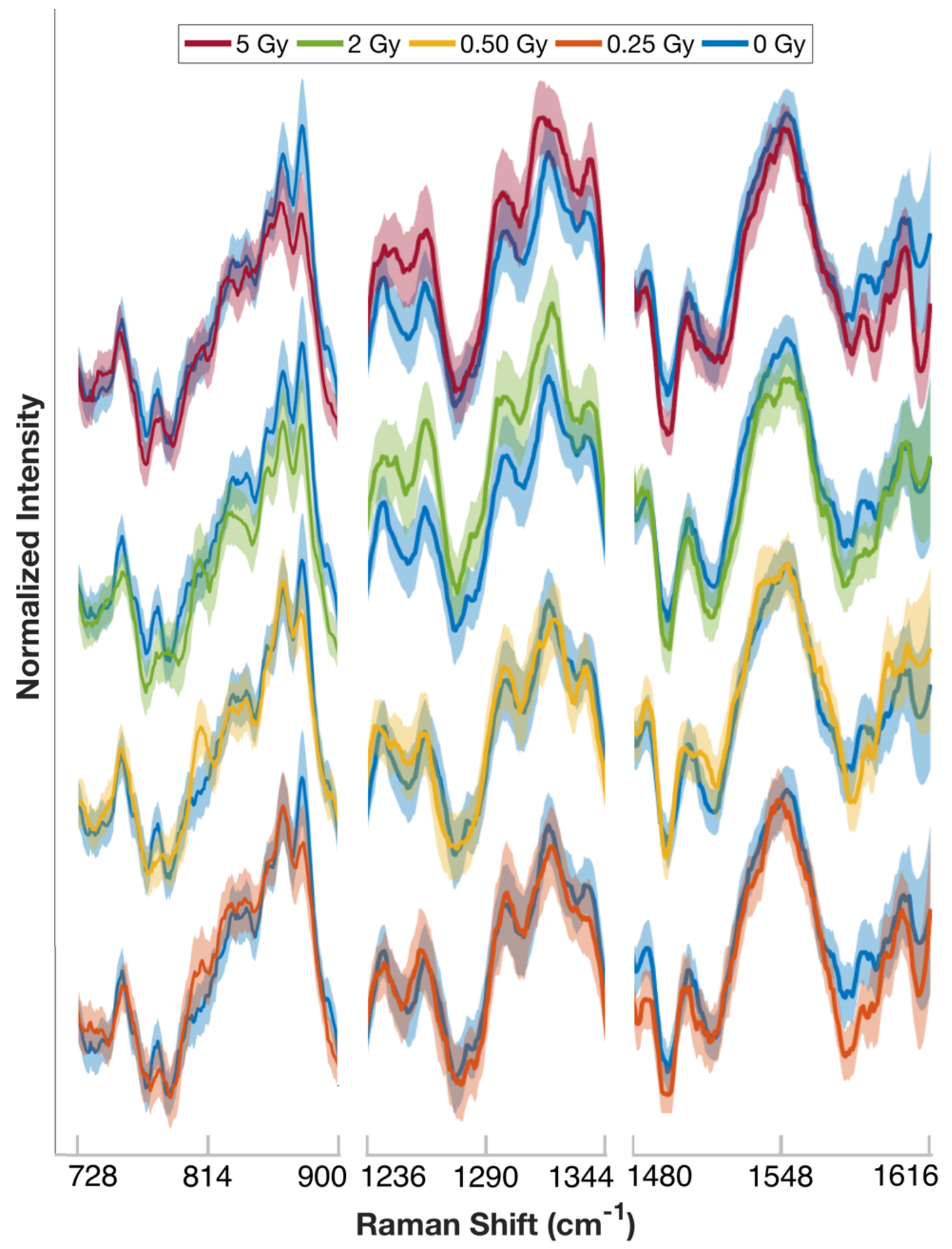

Figure 5.12: Zoomed-in view of some sections of the cytoplasm spectra in Figure 5.11. Note that these segments are from three spectral bands, and so the x-axis is discontinuous between segments. 


\subsection{PCA-LDA results}

\subsubsection{Principal Component Analysis}

Principal component analysis was used to reduce the dimensionality of the data by transforming the Raman spectra into uncorrelated variables, ordered by variance. The reduction is done by choosing the first $N$ PCs accounting for a majority of the variance, with the exact number chosen using some criterion. The criterion used in this study is given in section 4.6.2. The first 4 nucleus and cytoplasm PCs are plotted in Figures 5.13 and 5.14 respectively, for each dose-control comparison. Note that the first two principal components clearly correlate very well between all the dose-control comparisons, and less so for higher PCs.
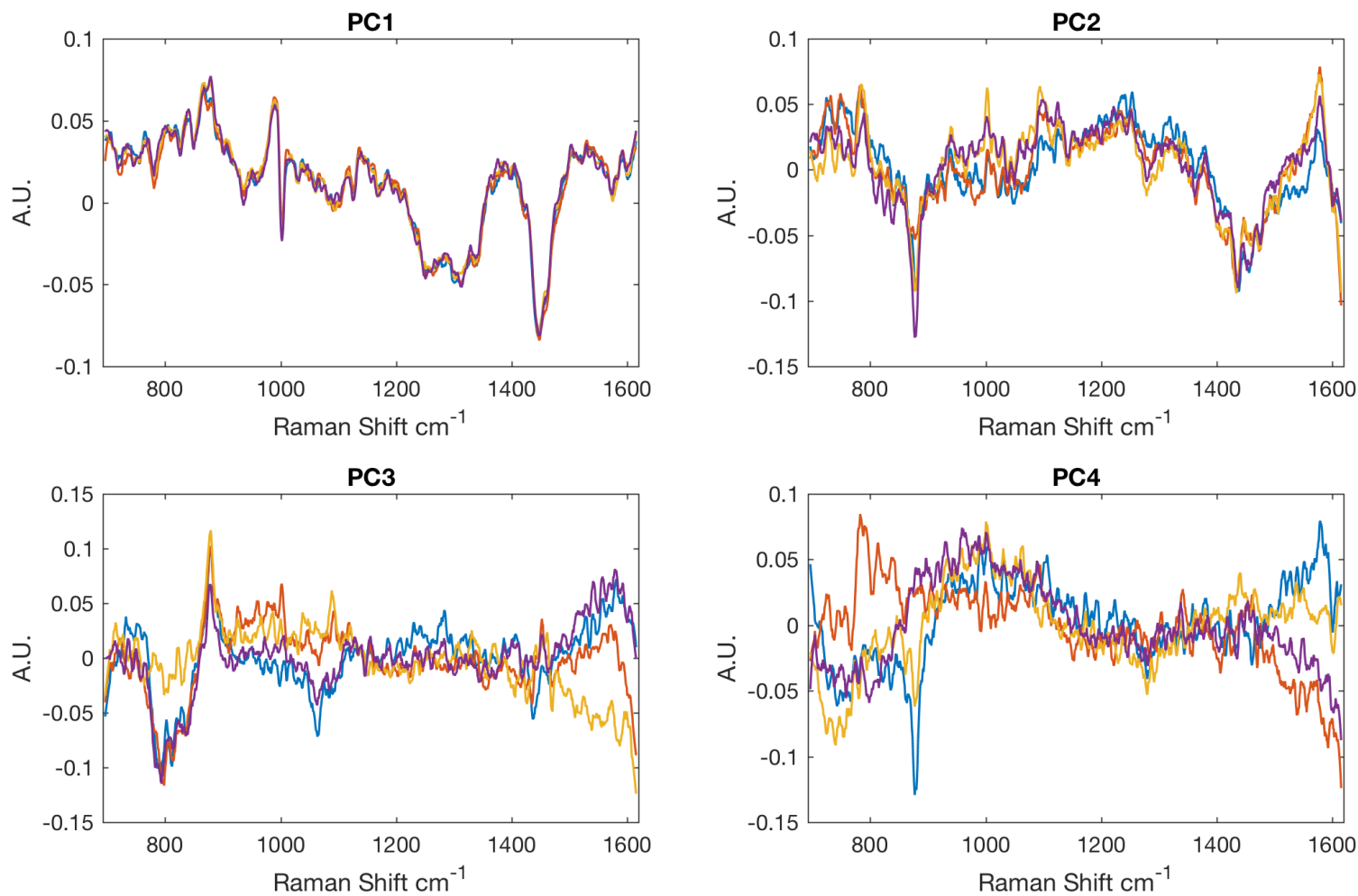

Figure 5.13: First 4 PCs for each nucleus dose-control pair. Blue is from 0.25 Gy, red is $0.5 \mathrm{~Gy}$, green is $2 \mathrm{~Gy}$, and purple is $5 \mathrm{~Gy}$. 

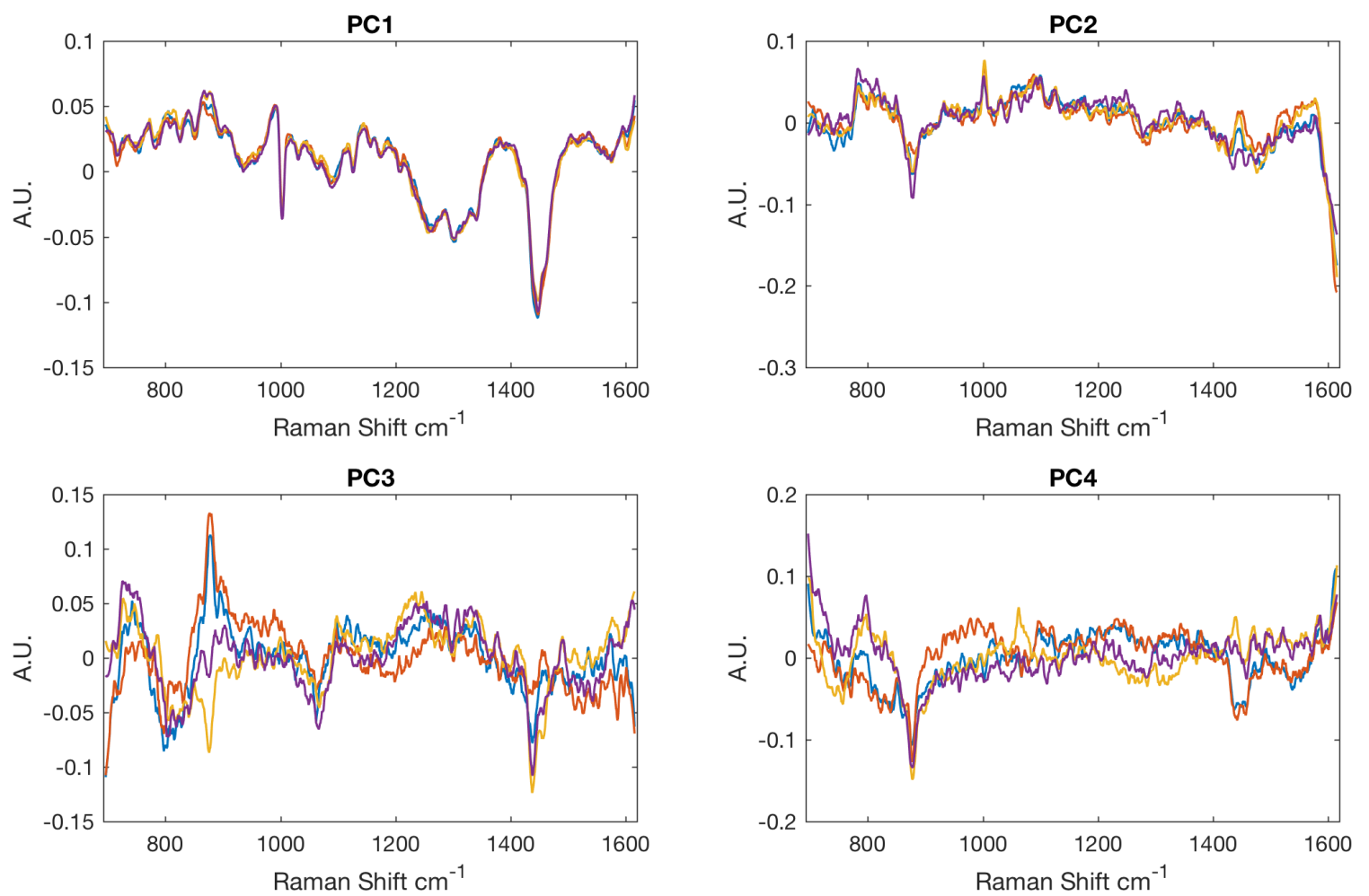

Figure 5.14: First 4 PCs for each cytoplasm dose-control pair. Blue is from 0.25 Gy, red is $0.5 \mathrm{~Gy}$, green is $2 \mathrm{~Gy}$, and purple is $5 \mathrm{~Gy}$.

\subsubsection{Contribution of cell cycle to spectra variance}

A visual inspection of the first 10 PCs for each analysis was performed to check for PCs that appear similar to those related to cell cycle, based on spectra plotted in Matthews et $a l^{65}$, and none were found to match that PC. Furthermore, measurement of cell cycle by Health Canada using Guava flow cytometry provided no statistically significant indication that the HLE cells were arrested following 5 Gy of irradiation within the 24-hour time frame, as seen in Appendix C.1. Therefore, the changes being seen in the Raman spectra are not related to cell cycle arrest. 


\subsubsection{Binary classification between each dose and control}

Discrimination between the control set and each dose was done using LDA of the PCA transformed spectra, with leave-one-out cross validation (LOOCV) used to estimate the accuracy of the classification model. Sensitivity, specificity, and accuracy results are given in Table 5.3.

Table 5.3: Binary classification LOOCV sensitivity, specificity, and accuracy results for nucleus and cytoplasm.

\begin{tabular}{ccccc}
\hline Nucleus & 0.25 Gy vs. 0 Gy & 0.5 Gy vs. 0 Gy & 2 Gy vs. 0 Gy & 5 Gy vs. 0 Gy \\
\hline Sensitivity & $81 \%$ & $73 \%$ & $76 \%$ & $81 \%$ \\
Specificity & $78 \%$ & $75 \%$ & $77 \%$ & $83 \%$ \\
Accuracy & $79 \%$ & $74 \%$ & $76 \%$ & $82 \%$ \\
\hline Cytoplasm & 0.25 Gy vs. 0 Gy & 0.5 Gy vs. 0 Gy & 2 Gy vs. 0 Gy & 5 Gy vs. 0 Gy \\
\hline Sensitivity & $73 \%$ & $84 \%$ & $75 \%$ & $86 \%$ \\
Specificity & $75 \%$ & $82 \%$ & $73 \%$ & $82 \%$ \\
Accuracy & $74 \%$ & $83 \%$ & $74 \%$ & $84 \%$ \\
\hline
\end{tabular}

As can be seen in Table 5.3, the minimum accuracy attained for any dose-control classification is $74 \%$. The number of PCs to use for each analysis was determined by performing the above analysis using 2 to $N$ PCs, where $N$ is the number of PCs for a given dose (68 for nucleus, 64 for cytoplasm), plotting the resulting accuracies, and choosing the "shoulder" so as to not include PCs that do not contribute significantly to discrimination, and most likely correspond to noise, as discussed in section 4.6.2.

Since LDA projects the data onto a 1-dimension line, by taking the dot product of the found LD with each spectrum in a given class, the resulting LD scores, which are the points along this line on which each spectrum is projected, form distributions of scores which correspond to the classes being discriminated. These can be plotted as histograms, as is done for each dose for nucleus data in Figure 5.15 and in Figure 5.16 for cytoplasm. The score distributions shown constructed from the tested data 
scores using LOOCV. The less overlap there is between the distributions, the higher the accuracy will be, with $100 \%$ accuracy only achievable when there is zero overlap.
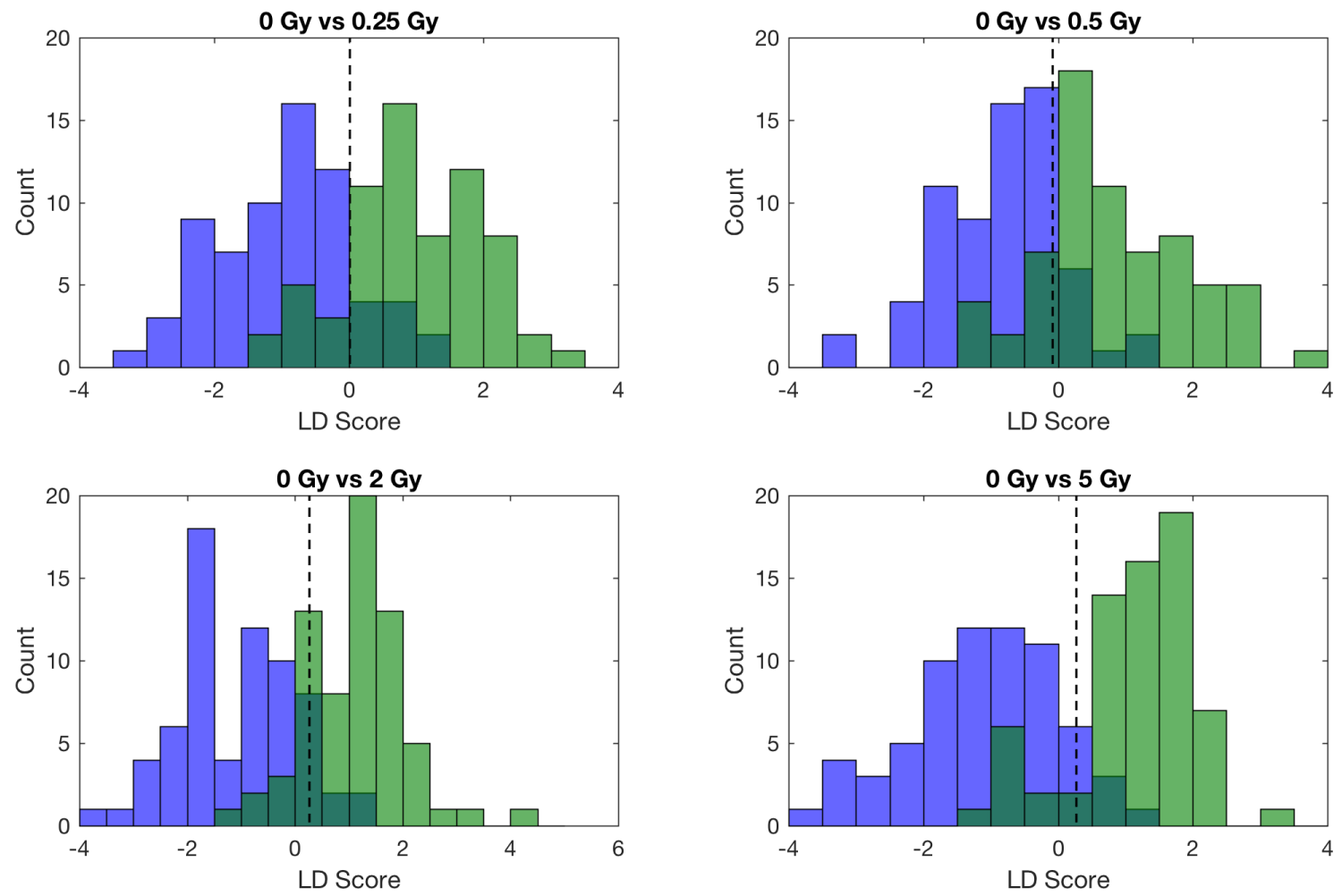

Figure 5.15: Nucleus LOOCV LD score distributions for each dose-control pair. Discrimination threshold indicated by vertical line. The control distribution is blue, the irradiated distribution is green, and overlapped regions are a dark blue-green. 

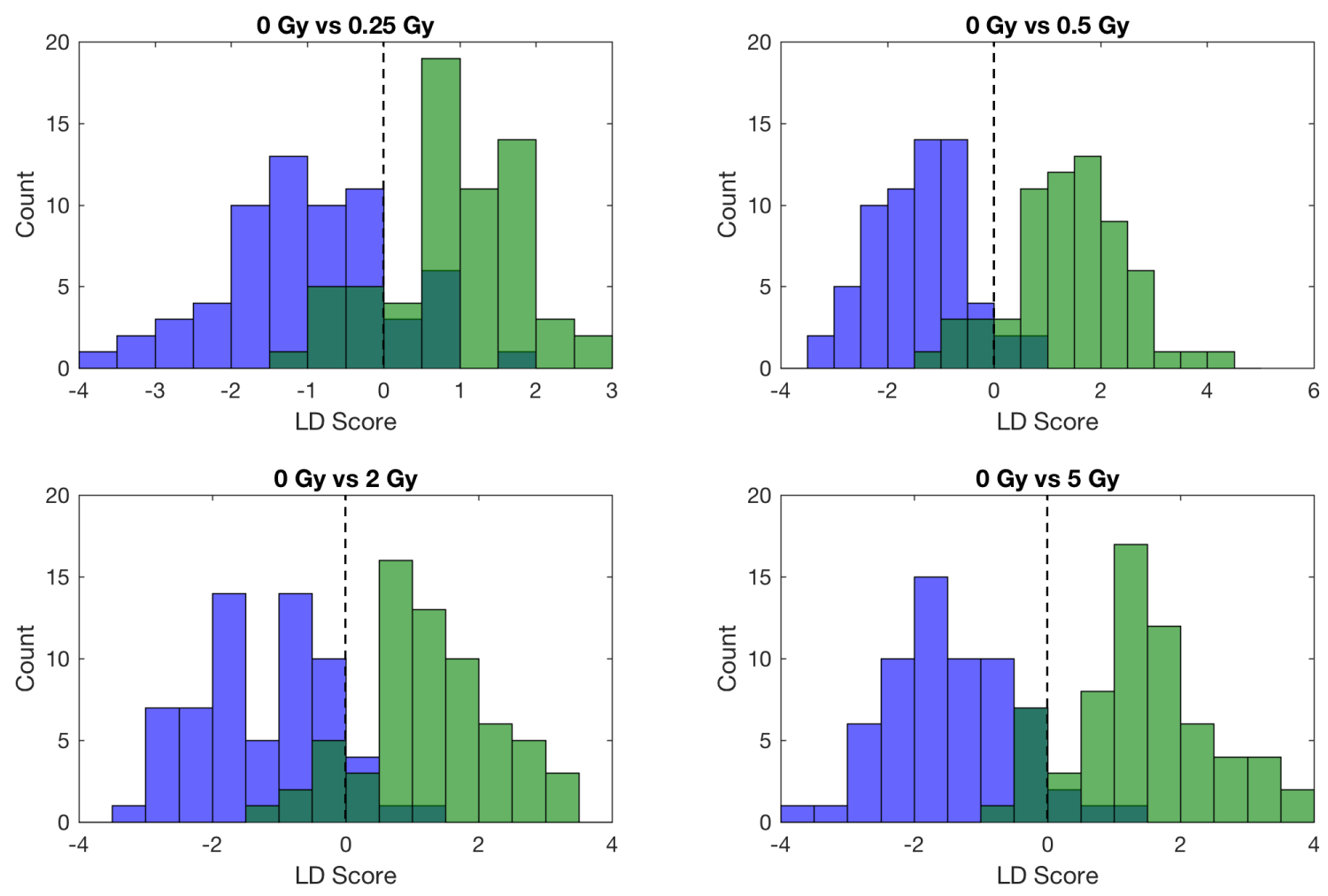

Figure 5.16: Cytoplasm LOOCV LD score distribution for each dose-control pair. Discrimination threshold indicated by vertical line. The control distribution is blue, the irradiated distribution is green, and overlapped regions are a dark blue-green.

\subsubsection{Analysis of Linear Discriminants and Molecular As- signments}

The linear discriminants (LDs) of each analysis were then transformed from PC space back to wavenumber space and used to determine which wavenumbers contributed most to each discrimination, based on the magnitude of the LD loadings for each wavenumber. This is described in detail in section 3.2.3. Before this was done, however, the LDs were visually compared to the difference spectra of each dose and control, as shown in Figures 5.17 and 5.18 for nucleus and Figures 5.19 and 5.20 for cytoplasm. 


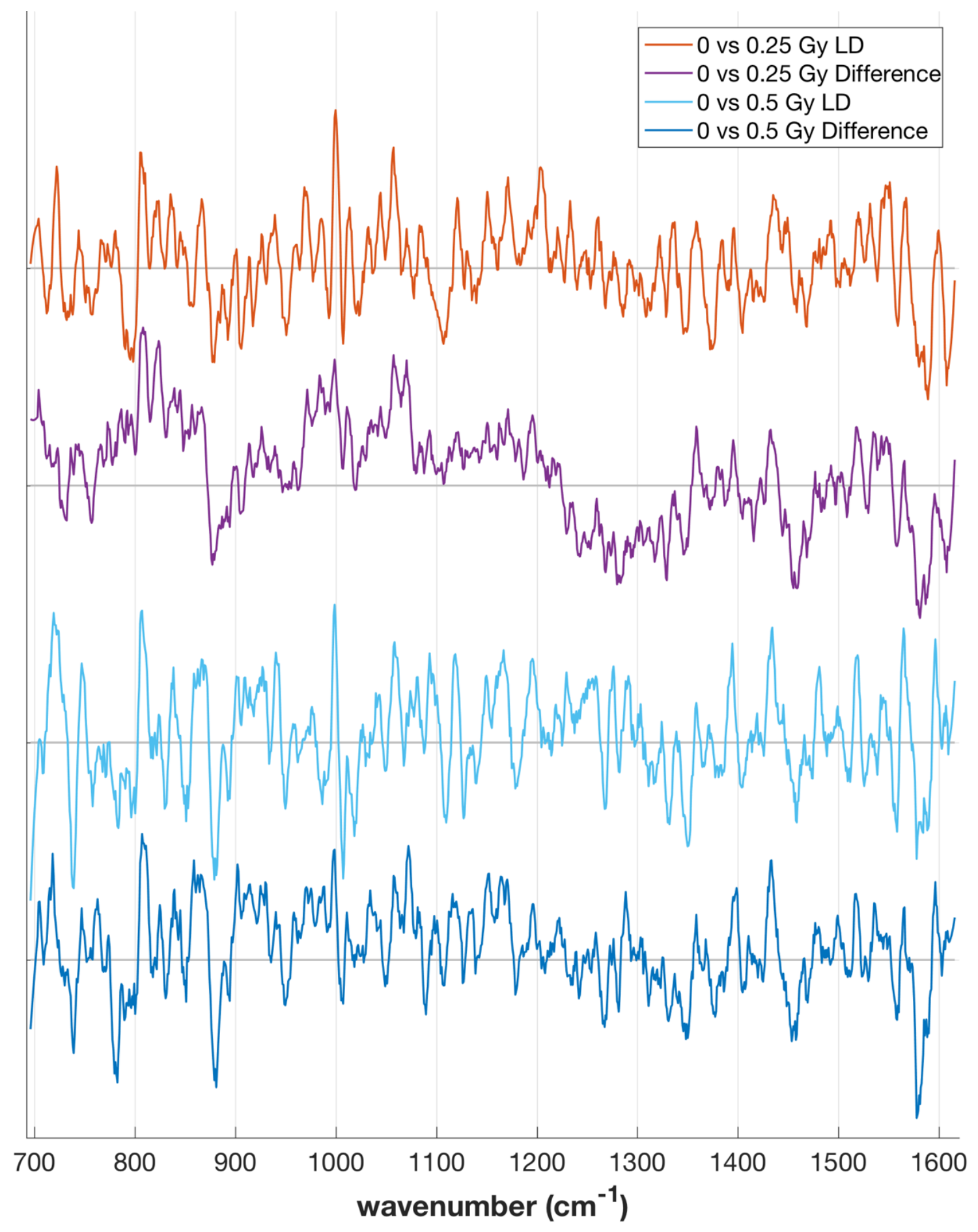

Figure 5.17: Linear discriminants and difference spectra from nucleus for 0 vs. 0.25 Gy and 0 vs. 0.5 Gy. 


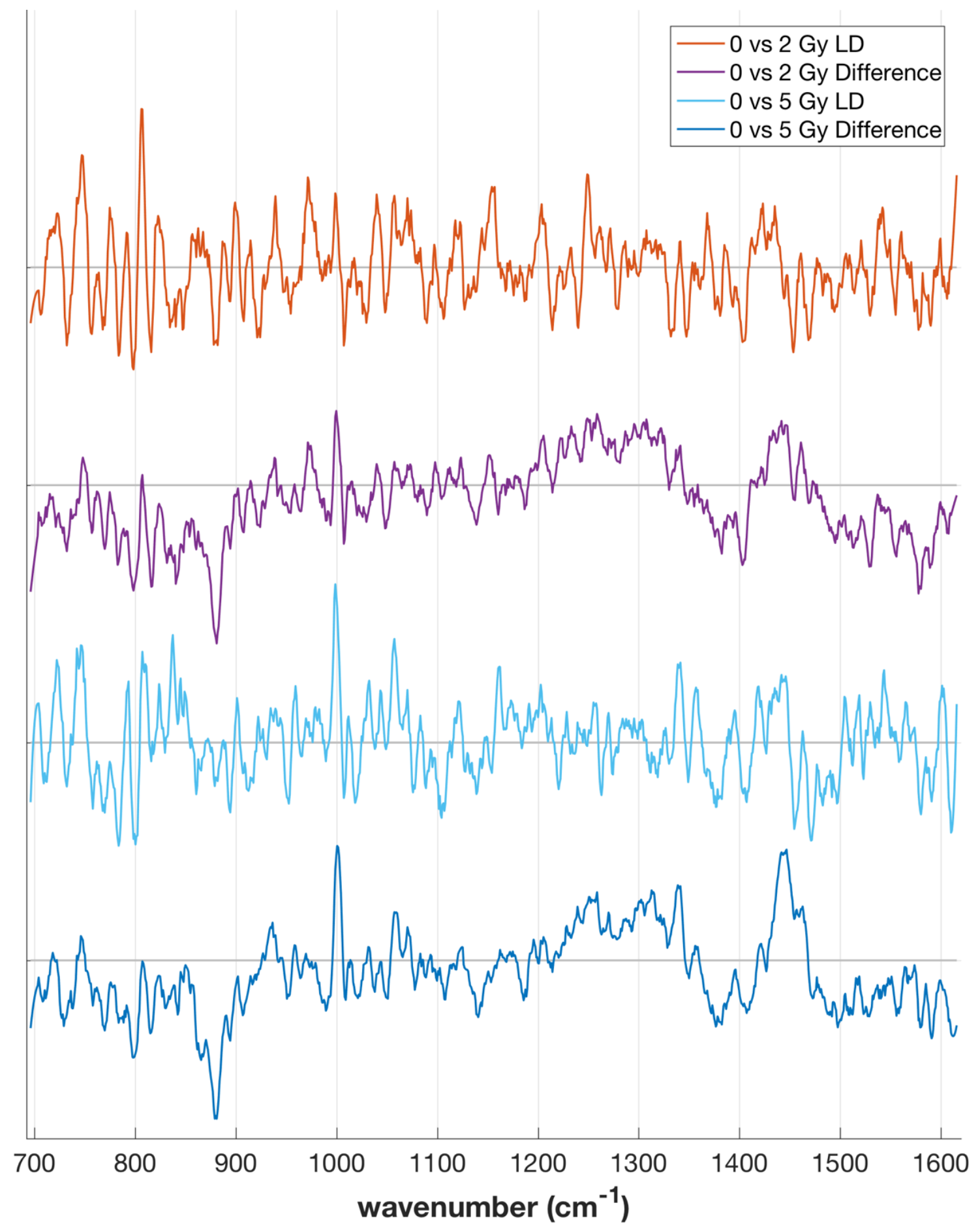

Figure 5.18: Linear discriminants and difference spectra from nucleus for 0 vs. 2 Gy and 0 vs. 5 Gy. 


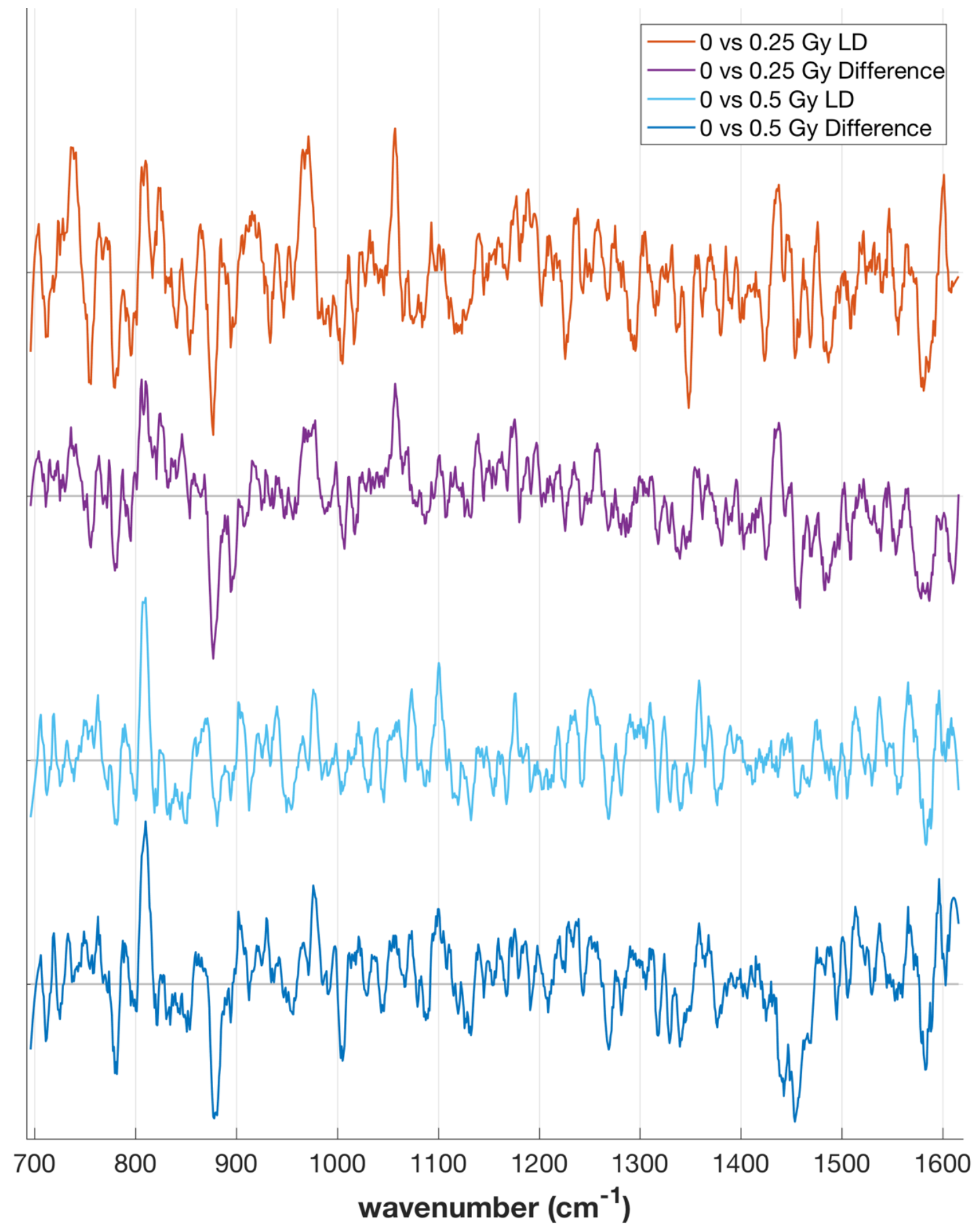

Figure 5.19: Linear discriminants and difference spectra from cytoplasm for 0 vs. 0.25 Gy and 0 vs. 0.5 Gy. 


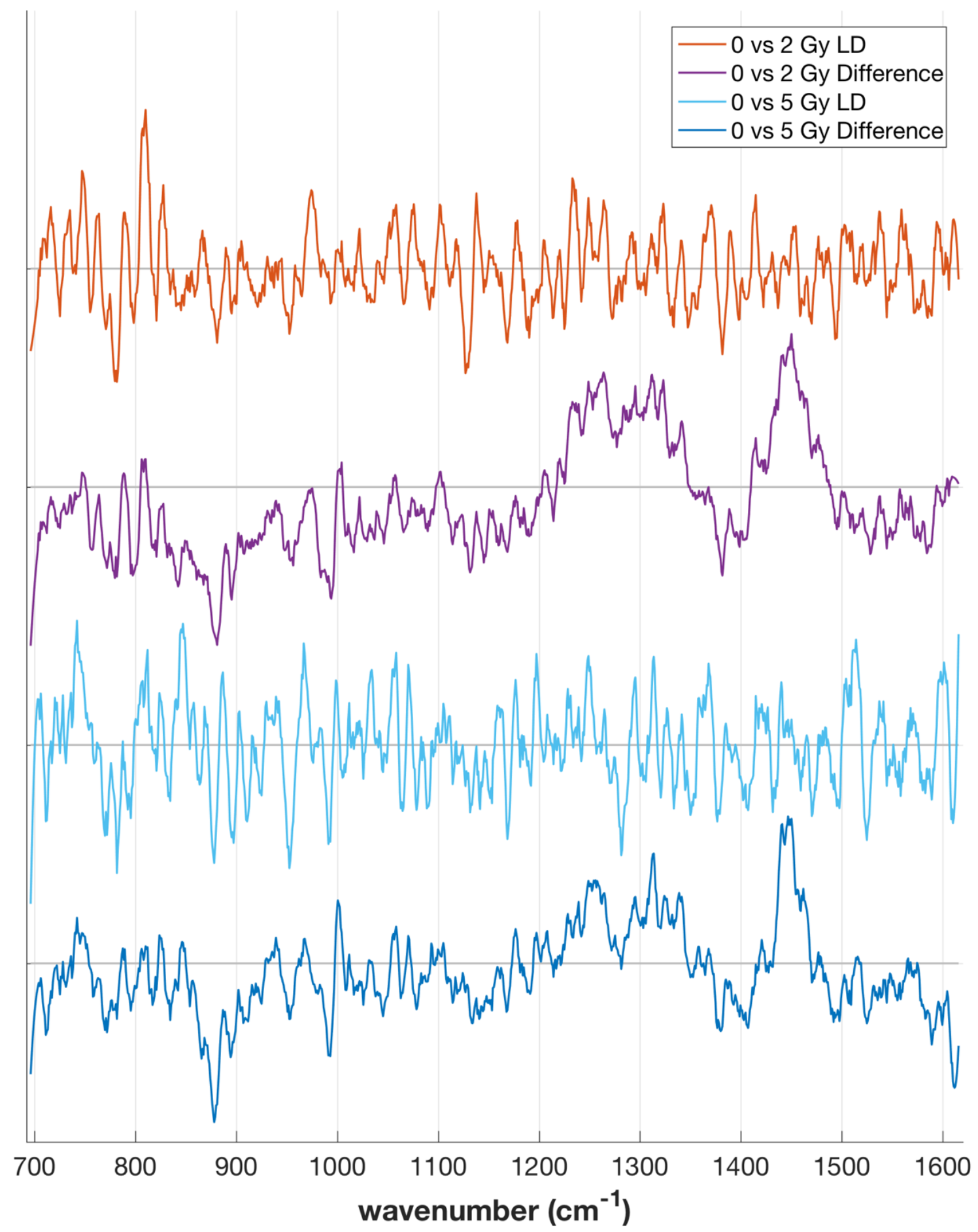

Figure 5.20: Linear discriminants and difference spectra from cytoplasm for 0 vs. 2 Gy and 0 vs. 5 Gy. 
These plots show that a given LD is quite similar to the mean difference spectrum between the two classes being discriminated. The fact that they show large differences as well, demonstrates that certain bands have more or less discriminatory value than others, regardless of whether or not that band difference is small or large. Some of the differences between LDs and mean difference spectra could also be explained by using a subset of PCs, but since the PCs with the highest variance were included, it seems unlikely that regions showing large mean differences would correspond to PCs with small variance. It is therefore more likely that the PCs which show these features were not weighted heavily in the LD loadings due to poor discriminatory value.

The most important Raman peaks and bands contributing to each analysis were then found by matching the most prominent LD loadings (by magnitude) to their corresponding Raman peaks in the mean HLE spectra for each dose. Since the LD score distributions were such that the control mean score was negative and the mean score for a given dose were always positive, and since positive LD loadings contribute to the positive dot product terms that sum to an LD score, it follows that they contribute more to a spectrum being classified as a given dose. Likewise, negative LD loadings contribute to the negative dot product terms that sum to an LD score, and so contribute more to a spectrum being classified as control.

Of the Raman peaks found using this method, those for which a statistically significant $(\mathrm{p}<0.05)$ difference between the means exists between a given dose and control are noted by an up or down arrow in Tables 5.4 and 5.5. The significance was determined using two-tailed Welch's t-test. An up-pointing arrow indicates a positive LD loading for that dose-control pair, and a downward pointing arrow indicates a negative one. Absence of an arrow indicates the Welch's t-test found a p-value of greater than $5 \%$, meaning the probability of seeing mean peak values that different from the same distribution was greater than $5 \%$. 
Table 5.4: Tentative Raman band assignments for LD Loadings (see Figures 5.17 and 5.18) from nucleus tabulating major peaks for each dose. The $\uparrow$ and $\downarrow$ arrows under each dose column indicate the sign of the LD loadings for that dose suggesting whether the molecules are more or less abundant in the irradiated cells compared to the control cells. Abbreviations: (A) adenine, (U) uracil, (T) thymine, (C) cytosine, $(\mathrm{G})$ guanine, (p) protein, (l) lipid, (s) saccharide, (m) metabolite, (d) DNA or RNA, (str) stretch, (bk) backbone, and (br) breathing. References are given by bracketed number.

\begin{tabular}{|c|c|c|c|c|c|}
\hline $\begin{array}{l}\text { Raman } \\
\text { Shift } \\
\left(\mathrm{cm}^{-1}\right)\end{array}$ & Molecular Assignment(s) & \multicolumn{4}{|c|}{$\begin{array}{l}\text { Change relative to } \\
\text { the control for var- } \\
\text { ious doses (Gy) }\end{array}$} \\
\hline & DNA/RNA & 0.25 & 0.5 & 2 & 5 \\
\hline 782 & $\mathrm{U}, \mathrm{C}, \mathrm{T}$ ring br; O-P-O str bk ${ }^{65,71}$ & $\uparrow$ & & $\downarrow$ & $\downarrow$ \\
\hline 1551 & $\mathrm{G}^{67}$ & $\uparrow$ & & & \\
\hline 1577 & $\mathrm{~A}, \mathrm{G}^{65,71}$ & & $\downarrow$ & & \\
\hline & Proteins/Amino Acids & & & & \\
\hline 747 & L-Phenylalanine ${ }^{67}$ & & & $\uparrow$ & $\uparrow$ \\
\hline 808 & Glutamate $^{67}$ & $\uparrow$ & $\uparrow$ & $\uparrow$ & $\uparrow$ \\
\hline 1056 & Glutamate $^{67}$ & $\uparrow$ & & & $\uparrow$ \\
\hline 1156 & $\mathrm{C}-\mathrm{C}, \mathrm{C}-\mathrm{N}$ str 67 & & & $\uparrow$ & \\
\hline 1204 & Amide III; $\mathrm{CH}_{2}$-glycine \& proline ${ }^{66}$ & $\uparrow$ & & & \\
\hline 1588 & Glycine; phenylalanine ${ }^{66}$ & $\downarrow$ & & & \\
\hline & Metabolites & & & & \\
\hline 799 & Acetyl coenzyme $\mathrm{A}^{67}$ & & & $\downarrow$ & $\downarrow$ \\
\hline 1580 & Acetoacetate ${ }^{65}$ & $\downarrow$ & & & \\
\hline 719 & $\begin{array}{l}\text { Mixed/Other } \\
\text { Choline (l) }\end{array}$ & & $\uparrow$ & & \\
\hline 723 & $\begin{array}{l}\text { Coenzyme A (m); Glutathione; }{ }^{67} \text { A } \\
(\mathrm{d})^{67}\end{array}$ & $\uparrow$ & $\uparrow$ & & \\
\hline 740 & $\mathrm{~T}(\mathrm{~d}) ;$ Glutamate $(\mathrm{p})^{67}$ & & $\downarrow$ & & \\
\hline 811 & O-P-O str RNA (d); Glutathione ${ }^{67}$ & $\uparrow$ & & & \\
\hline 838 & Trehalose (s) & & & & $\uparrow$ \\
\hline 879 & $\begin{array}{l}\text { Hydroxyproline (p); Tryptophan (p); }{ }^{66} \\
\text { Fucose }(\mathrm{s})^{67}\end{array}$ & $\downarrow$ & $\downarrow$ & $\downarrow$ & \\
\hline 971 & C (d); Oleic acid (l) & & & $\uparrow$ & \\
\hline 1250 & Amide III (p); G, C (d) ${ }^{66}$ & & & $\uparrow$ & \\
\hline 1403 & Glutathione $^{67}$ & & & $\downarrow$ & \\
\hline 1434 & Glutamate $(\mathrm{p})$; citric acid $(\mathrm{m})$ & & $\downarrow$ & & \\
\hline 1454 & Proline (p); myristic acid (l) & & & $\downarrow$ & $\downarrow$ \\
\hline
\end{tabular}


Table 5.5: Tentative Raman band assignments for LD Loadings (see Figures 5.19 and 5.20) from cytoplasm tabulating major peaks for each dose. The $\uparrow$ and $\downarrow$ arrows under each dose column indicate the sign of the LD loadings for that dose suggesting whether the molecules are more or less abundant in the irradiated cells compared to the control cells. Abbreviations: (A) adenine, (U) uracil, (T) thymine, (C) cytosine, $(\mathrm{G})$ guanine, (p) protein, (l) lipid, (s) saccharide, (m) metabolite, (d) DNA or RNA, (str) stretch, (bk) backbone, and (br) breathing. References are given by bracketed number.

\begin{tabular}{|c|c|c|c|c|c|}
\hline $\begin{array}{l}\text { Raman } \\
\text { Shift } \\
\left(\mathrm{cm}^{-1}\right)\end{array}$ & Molecular Assignment(s) & \multicolumn{4}{|c|}{$\begin{array}{l}\text { Change relative to } \\
\text { the control for var- } \\
\text { ious doses }(\mathrm{Gy})\end{array}$} \\
\hline & DNA/RNA & 0.25 & 0.5 & 2 & 5 \\
\hline 782 & $\mathrm{U}, \mathrm{C}, \mathrm{T}$ ring br; O-P-O str bk ${ }^{65,71}$ & & $\downarrow$ & $\downarrow$ & $\downarrow$ \\
\hline & Proteins/Amino Acids & & & & \\
\hline 747 & L-Phenylalanine $^{67}$ & & & $\uparrow$ & \\
\hline 756 & Tryptophan $(p)^{66}$ & $\downarrow$ & & & \\
\hline 778 & Tryptophan ${ }^{67}$ & $\downarrow$ & & & \\
\hline 881 & Tryptophan ${ }^{66}$ & & $\downarrow$ & $\downarrow$ & \\
\hline 1056 & Glutamate $^{67}$ & $\uparrow$ & & & $\uparrow$ \\
\hline 1567 & Valine $(\mathrm{p})^{67}$ & & $\uparrow$ & & \\
\hline 1583 & $\mathrm{C}-\mathrm{C}$ bending mode of phenylalanine ${ }^{66}$ & & $\uparrow$ & & \\
\hline & Mixed/Other & & & & \\
\hline 740 & $\mathrm{~T}(\mathrm{~d})$; Glutamate $(\mathrm{p})^{67}$ & $\uparrow$ & & & \\
\hline 811 & O-P-O str RNA (d); Glutathione ${ }^{67}$ & $\uparrow$ & $\uparrow$ & $\uparrow$ & \\
\hline 828 & Tyrosine $(\mathrm{p}),{ }^{66}$ gluathione ${ }^{67}$ & & & $\uparrow$ & \\
\hline 879 & $\begin{array}{l}\text { Hydroxyproline }(\mathrm{p}) \text {; Tryptophan }(\mathrm{p}){ }^{66} \\
\text { Coenzyme A }(\mathrm{m}) \text {, Fucose }(\mathrm{s})^{67}\end{array}$ & $\downarrow$ & & & $\downarrow$ \\
\hline 953 & Glutathione; lactose $(\mathrm{s})^{67}$ & & & & $\downarrow$ \\
\hline 971 & C (d); Oleic acid (l) & $\uparrow$ & & & \\
\hline 1127 & Lauric acid (l), Amylopectin (s) & & & $\downarrow$ & \\
\hline 1234 & G (d); Glutathione; ${ }^{67}$ Amide III ${ }^{66}$ & & & $\uparrow$ & \\
\hline 1281 & Glutathione $^{67}$ & & & & $\downarrow$ \\
\hline 1359 & Alanine $(\mathrm{p}) ;$ lactose $(\mathrm{s})$ & & & $\uparrow$ & \\
\hline 1415 & Glutathione $^{67}$ & & & $\uparrow$ & \\
\hline 1580 & Acetoacetate $(\mathrm{m})$ & $\downarrow$ & & & \\
\hline
\end{tabular}


Examining the molecular assignments and LD loading directions for the nucleus spectra in Table 5.4, we see from Raman shifts $782 \mathrm{~cm}^{-1}$, for 2 Gy and 5 Gy, and $1577 \mathrm{~cm}^{-1}$, for 0.5 Gy that the nucleobases are generally decreasing, with the exception of guanine, which shows a low dose increase at $1551 \mathrm{~cm}^{-1}$ for $0.25 \mathrm{~Gy}$. It is not clear why the change in a nucleobase would be different for a lower dose. An increase in glutamate is seen increasing for every dose at $808 \mathrm{~cm}^{-1}$, while at $747 \mathrm{~cm}^{-1}$, we have indications of phenylalanine increasing for 2 and 5 Gy. Peaks corresponding to metabolites, such as acetyl coenzyme A and acetoacetate, only show decreases. Among peaks with mixed assignments, or those that do not fall under DNA/RNA or protein/amino acid categories, we see the peak at $879 \mathrm{~cm}^{-1}$, which corresponds to hydroxyproline, tryptophan, coenzyme A, and fucose, decreasing for $0.25,0.5$, and 2 Gy.

Table 5.5, which shows molecular assignments and LD signs for the cytoplasm spectra, we see a decrease in nucleic acids for $0.5,2$, and $5 \mathrm{~Gy}$, at $782 \mathrm{~cm}^{-1}$. While glutamate $\left(1056 \mathrm{~cm}^{-1}\right)$ seems to be increasing in this case as well, the Raman shifts

$756 \mathrm{~cm}^{-1}, 778 \mathrm{~cm}^{-1}$, and $881 \mathrm{~cm}^{-1}$ suggest the amino acid tryptophan is decreasing for doses up to 2 Gy. Although it is a shared assignment with RNA at Raman shift $811 \mathrm{~cm}^{-1}$, glutathione might be showing increases at $0.25,0.5$, and 2 Gy. Its decrease might also be indicated by the shared assignment at $828 \mathrm{~cm}^{-1}$. The decrease of glutathione at $1281 \mathrm{~cm}^{-1}$ for 5 Gy suggests that the higher dose might be saturating the antioxidant, and even depleting it.

\subsection{Ultra-low dose response}

Some of the batches from which Raman spectra were collected included 2 extra sets of HLE cells irradiated at ultra-low doses of 0.01 and 0.05 Gy. Since these doses were not included for every batch, they were excluded from the binary classification in 
order to maximize sample sizes without having class size inequalities. These datasets were included in an analysis of how the area under a given peak changed relative to dose, which was looked at for several of the peaks that were found to be significant in the binary classification. This analysis was inspired by the paper by Meade et $a l^{25}$, and looks at the absolute difference of the area under the curve relative to control. The metric used is given by Equation 4.6, and give a value between 0 and 1, with a value closer to 0 indicating a large difference in area under the peak relative to control, and 1 indicating no difference.

The generated data were then fit to the induced repair model of cell survival given by Equation 2.8, as was a clonogenic assay $(\mathrm{N}=3)$ performed by Dr. Sami Qutob at Health Canada. In the case of the assay, and several of the peaks, particularly those corresponding to tryptophan at $879 \mathrm{~cm}^{-1}$, glutathione and/or RNA at $811 \mathrm{~cm}^{-1}$, DNA base pairs at $782 \mathrm{~cm}^{-1}$, and glutamate at $1056 \mathrm{~cm}^{-1}$, a close fit was found for each, and a coefficient of determination calculated to determine the goodness of fit. The best fits are shown in Figures 5.21 a), b), c), and d). A clonogenic assay (n=3) with a induced repair survival curve fit is shown in figure 5.22. The best fits (high and low) using the linear quadratic model are also shown for the clonogenic assay results. Note that the fits found from the Raman data are from the nucleus data only. All fits also had an F-test $\mathrm{p}$-value of $\mathrm{p}<0.05$, using the normal survival curve model as comparison.

The $R^{2}$ ranged from 0.76 to 0.93 from the Raman data, and 0.97 for the clonogenic survival assay, showing that the induced repair model given by Equation 2.8 shows very good agreement with the data for these peaks and with the clonogenic assay data. Examining the curves themselves, they show a large drop between control and $0.5 \mathrm{~Gy}$, with a minimum close to $0.25 \mathrm{~Gy}$, rising up to a local maximum at 1 Gy (note, no data points at $1 \mathrm{~Gy}$ ), and then either little change or a slow tapering off towards 5 Gy. For the clonogenic survival assay, the tapering off is steeper than 


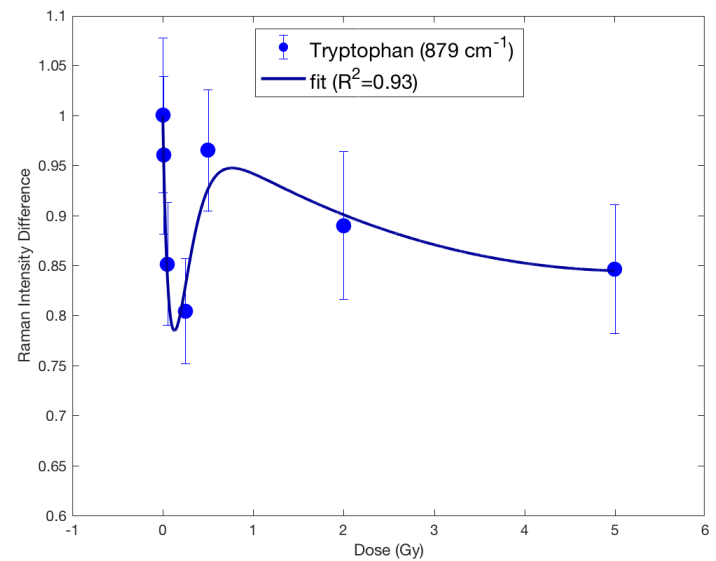

(a)

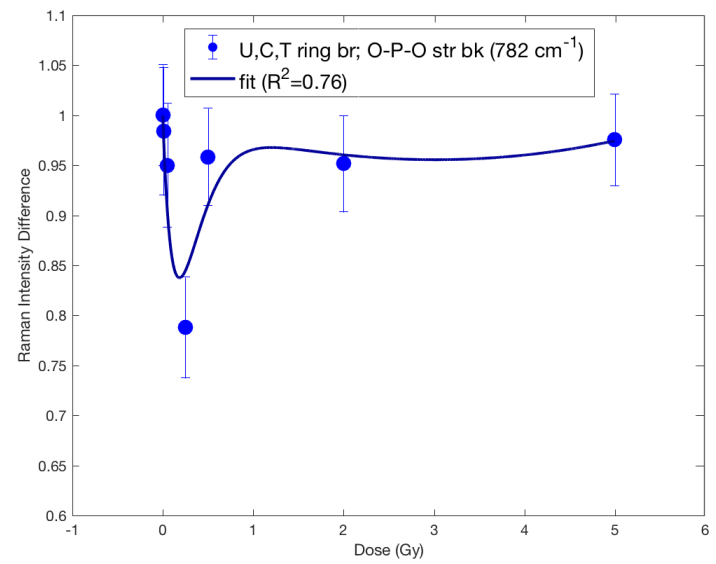

(c)

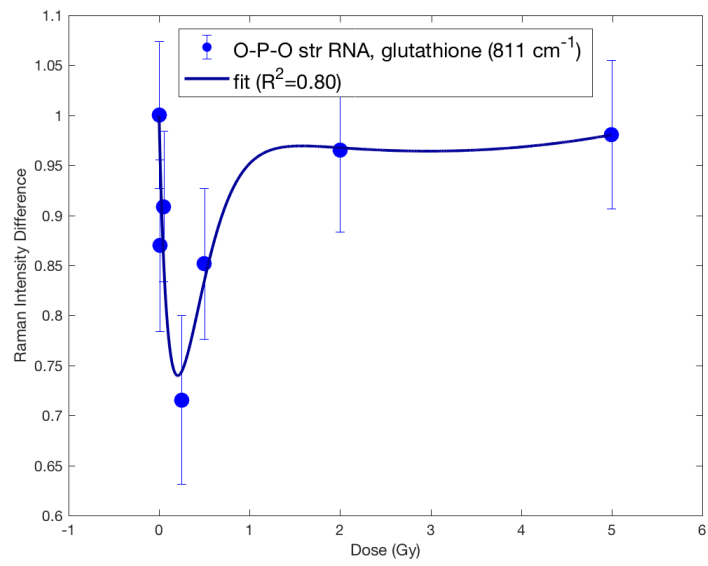

(b)

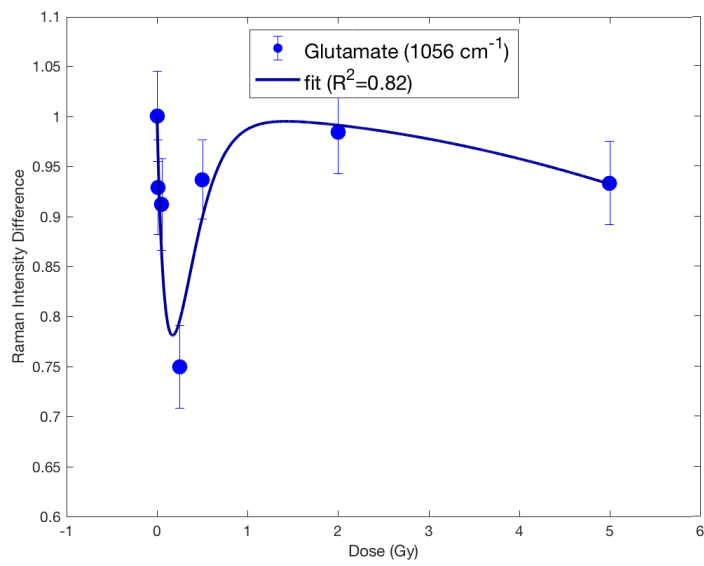

(d)

Figure 5.21: Raman band area absolute difference ratios, including ultra-low doses 0.01 and $0.05 \mathrm{~Gy}$, corresponding to a) tryptophan and hydroxyproline at $879 \mathrm{~cm}^{-1}$, b) O-P-O stretching vibration of RNA and glutathione at $811 \mathrm{~cm}^{-1}$, c) U, C, and T ring breathing modes and O-P-O backbone stretching of DNA at $782 \mathrm{~cm}^{-1}$, and d) glutamate at $1056 \mathrm{~cm}^{-1}$. A curve based on the induced repair model is fitted to the data.

the spectral band area absolute difference ratios, which is unsurprising since it corresponds to actual cell survival, and not metabolic changes. This sudden drop in cell count, followed by a slower decrease, is consistent with a transition between the low-dose hypersensitive region and an induction of radio-resistance, which induced repair models of cell survival presume. 


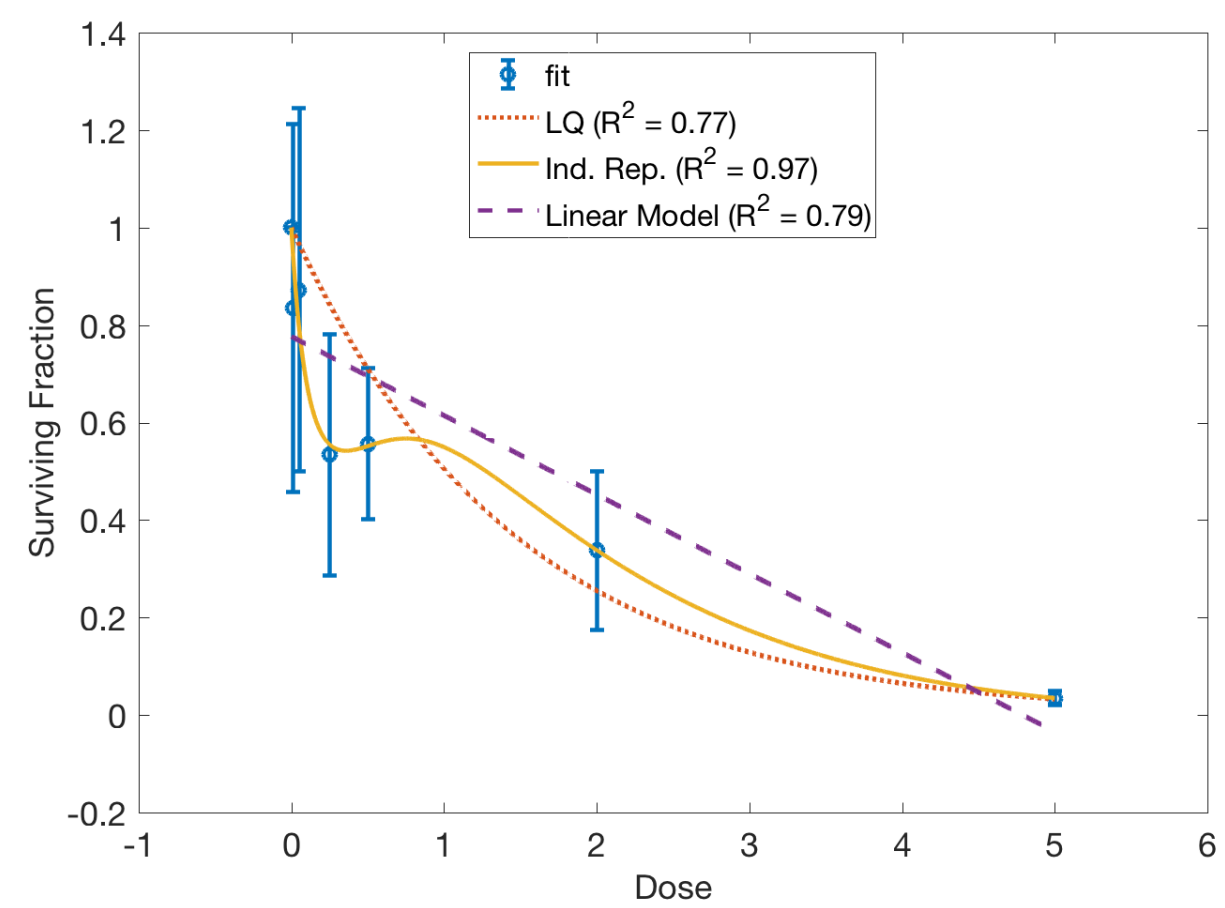

(a) 5

Figure 5.22: Surviving fraction of HLE cells as a function of dose from clonogenic survival assay $(\mathrm{n}=3)$, and with induced repair model fit as well as LQ fit and a simple linear fit. 


\section{Chapter 6}

\section{Instrumentation upgrade}

\subsection{Limitations of previous apparatus}

The previous Raman microscope had certain limitations when it comes to acquiring a large number of measurements. While the general description of how the previous setup worked, and what performance it was capable of, were discussed in sections 4.1 and 4.2 respectively, in this section the limitations of the CRM, particularly in regards to throughput, will be discussed. While its ability to target micron-sized structures is useful for investigating biomolecular differences in different cellular regions, it is less useful when attempting to acquire spectra that characterize the nucleus or cell as a whole. For discriminating between cells irradiated at different doses, the spectrum representing the cell should be a mean spectrum of the whole cell to avoid heterogeneities. The spectrum collected will then better represent the cell as a whole, and allow for comparisons to other populations of cells. Collecting spectra from several cells at once could also be useful for dosimetric estimates, where the spectrum represents a region of tissue rather than an individual cell. 


\subsubsection{Spot size and grid measurements}

The experiment performed on HLE cells attempted to extract a mean spectrum representative of each cell as a whole, while still falling within experimental time constraints. While the ability to target micron-sized structures is useful for investigating biomolecular differences in different cellular regions, it is less useful when attempting to acquire spectra that characterize the nucleus or cell as a whole. For discriminating between cells irradiated at different doses, the spectrum representing the cell should be a mean spectrum of the whole cell to avoid intracellular hetrogeneities. The spectrum collected will then better represent the cell as a whole, and allow for comparisons to other populations of cells. Collecting spectra from a several cells at once could also be useful for biodosimetry, where the spectrum represents a region of tissue rather than an individual cell. This latter point may be important for future experiments investigating cells irradiated at extremely low doses, as work simulating dose deposition on cells spread over a surface suggests the variance in dose between cells becomes significant. ${ }^{72}$

Thus, one way to improve the apparatus for experiments in which a spectrum representative of the whole cell, or a cluster of cells, is to have a larger spot size, or have the spot spread out as a line. In the case of line focussed laser spot, it could be $1 \mu \mathrm{m}$ in one axis and $25 \mu \mathrm{m}$ in the other. A spectrum representative of the cell or cluster could then be acquired by moving the line $1 \mu \mathrm{m}$ at a time across the cell, and taking a spectrum at each increment. While this would not necessarily decrease acquisition time, it would ensure that the sampled regions of the cell cover most of it, rather than a select few points. This should decrease the contribution of intracellular variability to the final averaged spectrum for that cell. A large enough circular spot size, say $25 \mu \mathrm{m}$ in diameter, would be even simpler in that the laser spot would not have to be moved. 


\subsubsection{Laser power}

The simplest way to improve the signal-to-noise ratio (SNR) of a Raman microscope is to increase the number of Raman scattered photons by increasing the laser power. Generally speaking, the Raman signal intensity detected, $S$, will be proportional to the number of Raman scattered photons, which in turn is proportional to the number of incident photons, $N$, from the laser, and so increasing the laser intensity will increase signal intensity. This will be the case for intensities below the photodamage and thermal damage thresholds of the sample. Ideally, the number of background photons, where background means non-Raman scattered light detected from sources such as fluorescence and Rayleigh scattered light that makes it through the filters, will also be proportional to the number of incident photons. In such a case, the background intensity will follow Poisson statistics, and so the amount of noise it contributes to the signal, $\sigma_{B}$, should be equal to the square root of the background intensity. ${ }^{54}$ This is true for the noise inherent in the Raman signal, given by $\sigma_{S}$, as well. Hence, the Raman signal-to-noise ratio (SNR) can be described by

$$
\begin{aligned}
S N R & =\frac{S}{\sqrt{\sigma_{S}^{2}+\sigma_{B}^{2}+\sigma_{d}^{2}+\sigma_{F}^{2}+\sigma_{r}^{2}}} \\
& =\frac{\alpha N}{\sqrt{\alpha N+\beta N+\sigma_{d}^{2}+\sigma_{F}^{2}+\sigma_{r}^{2}}}
\end{aligned}
$$

where $\alpha$ is the constant of proportionality for the Raman signal to the number of incident laser photons, and $\beta$ is the same for background sources (e.g. fluorescence), $\sigma_{d}$ is the standard deviation of the dark current, $\sigma_{F}$ is flicker noise from variation in laser intensity, and $\sigma_{r}$ is readout noise. ${ }^{54}$ Since $\sigma_{d}$, and $\sigma_{r}$ are independent of $N$, and $\sigma_{F}$ is negligible for multichannel spectrometers (i.e. those using CCDs rather than scanning through wavelengths onto a photomultiplier tube) and long acquisition times, it follows that increasing $N$ will increase the SNR. Thus, a Raman signal acquired using a higher laser power should have a better signal-to-noise ratio than one 
found using a lower laser power for the same acquisition time, since the corresponding number of incident photons on the sample will be greater.

\subsubsection{Confocality losses}

While confocality is useful for when one wishes to sample only from a small focal volume, and thus allow for high specificity while also reducing contributions to background from surrounding materials, such as the quartz cover slip, it does incur signal loss. This loss is unimportant for HLE cells grown thinly on surface, but for spheroid cells grown in solution, Raman signal from above and below the targeted volume may still be within the cell.

Additionally, if the cells are pelleted, that is, condensed into a tightly packed bunch using a centrifuge, then the sample depth will be much greater than the volume sampled by a confocal microscope. Thus, with confocality removed, a much larger signal can be collected from such a sample. Quartz contributions to background can still be minimized, in fact even more so, since the height of the pellet from the quartz surface can be much greater than the objective's depth of focus.

\subsubsection{Brightfield imaging}

One issue the previous CRM had that reduced throughput but which does not relate to system performance is how brightfield images were acquired. The light path from the sample to the brightfield camera required a mirror be placed above the main dichroic. This had to be done manually, and the mirror rotated such that the image reflected $90^{\circ}$ to the left and reached the brightfield camera. Since new cells could only be targeted while imaging the brightfield, and since images of the starting point and end point of the $3 \times 3$ grid had to be recorded to ensure measurements began and ended 
where they should, this meant that the procedure of removing the mirror and then placing it back in place had to be performed for every measurement. This procedure had to be done carefully as well, since the mirror was not far from the main dichroic, a slight nudge on which would through off the alignment and ruin the experiment for the day.

\subsubsection{Modularity}

While more modular than a commercial Raman microscope, due to its being built on optical breadboard and using a cage system with standard $1^{\prime \prime}$ optical components, the previous setup was not as modular as it could be. Cage systems are convenient for a fixed optical path in which optical elements such as lenses, filters, mirrors, and apertures might be placed. They are not flexible for when one wishes to change things. Changing a path within a cage system requires disassembling and reassembling that portion, and typically realigning the entire setup afterwards. Being a free-space optical path, it relies on mirrors that have to be precisely aligned, and this alignment can drift over time. The more mirrors there are in an apparatus, the more variables affecting the alignment there are and the longer a realignment will take.

Fiber optic connections have advantages in this regard. The optical path is simply along the fiber, and as long as the fiber is not bent beyond a certain limit, this path can be quickly changed without disturbing system performance. The only parts sensitive to change are the fiber coupling points, but these can be made modular as well using a short cage segment with lenses and fiber couples placed in their optimal position. Bundled fibers can be used as well to shape the light output on one end in ways difficult to do using lenses and mirrors. The main downside of using fibers in any system is that the losses will tend to be higher than a free-space setup since the fiber will attenuate light more than air, and coupling can be quite difficult for 
some small core diameter (e.g. $9 \mu \mathrm{m}$, single mode fibers). A modular setup using fibers can allow for quickly swapping between fiber coupled different lasers, different fiber coupled spectrometers, and can even be made mobile by stacking the different components in a cart. For these reasons, having a modular setup using optical fibers is highly desirable.

\subsection{Raman microscope version 2}

A schematic of the new Carleton Raman Microscope, CRM2, is shown in Figure 6.1. The changes made from the previous setup were all to address the limitations mentioned.

As can be seen in Figure 6.1, the total light path is with respect to laser and signal is greatly simplified. The brightfield light path is more complicated, but since this has no impact on system performance with respect to data acquisition, it is of less concern. The main changes made are outlined in the following sections. Note that the bottom two mirrors shown in the schematic are simplified from the actual apparatus, with the brightfield light path needing to be first sent in the out-of-page direction and then upward. This is because the LED is actually in a plane behind the main plane of the apparatus. The spectrometer is not shown but it is the same Shamrock SR-303i as described in section 4.1.2.

The main light paths are as follows: the laser light travels from the laser through fiber 1 which is coupled to a cage system. When exiting the fiber, the laser light is collimated by L1, a $40 \mathrm{~mm}$ focal length plano-convex lens. The collimated laser light then passes through a bandpass filter centered at the laser wavelength of $785 \mathrm{~nm}$. This filters out any fluorescence from the fiber at wavelengths above and below this value. This was found to be necessary when testing the first build of the apparatus. 


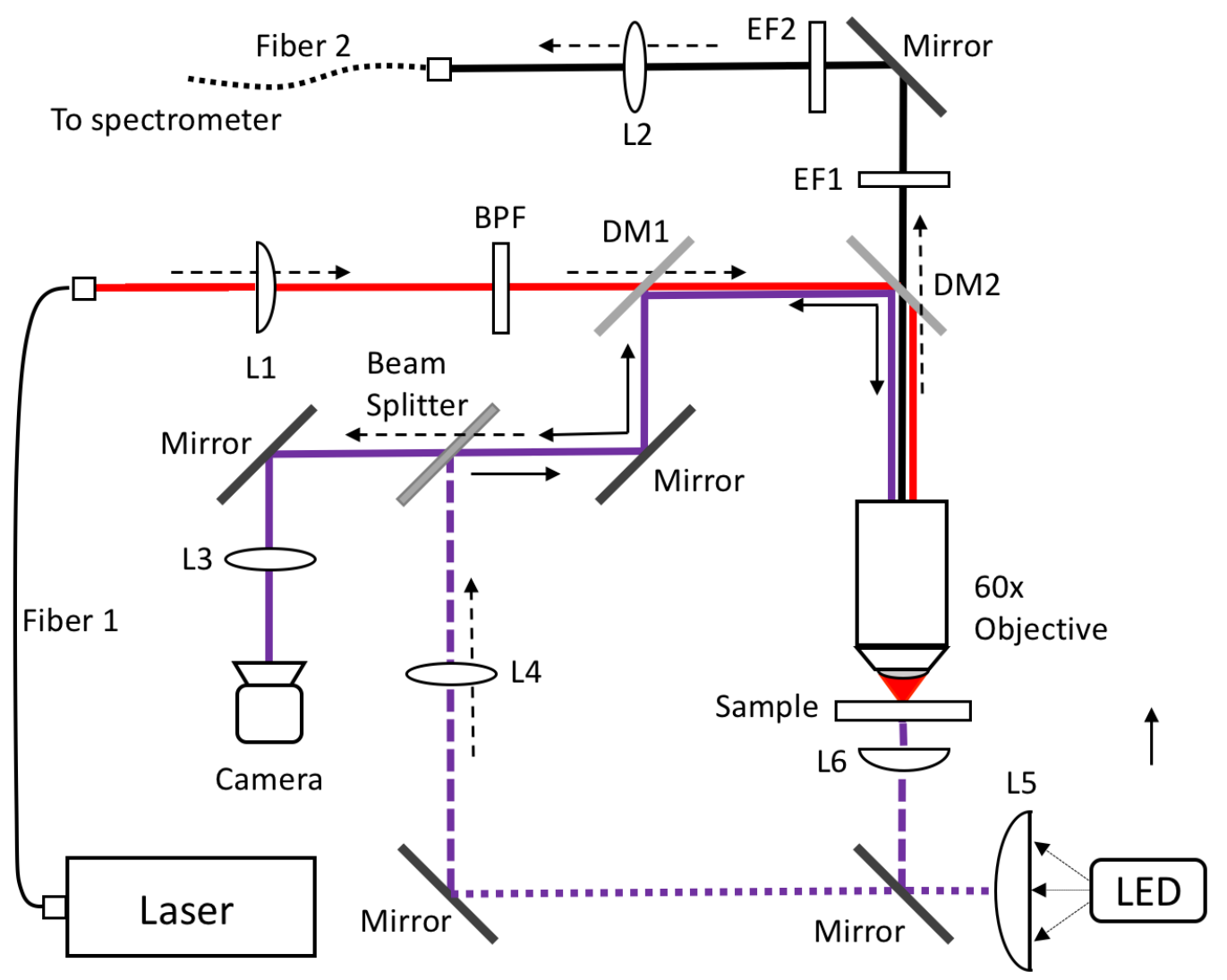

Figure 6.1: Schematic of newly built Carleton Raman Microscope version 2 (CRM2). $\mathrm{L}$ is for lens, $\mathrm{BPF}$ is for bandpass filter, DM is for dichroic mirror, and $\mathrm{EF}$ is for edge filter.

From there, the laser light passes through the first dichroic mirror, DM1, an Iridian short-pass DM that reflects any below $750 \mathrm{~nm}$, and hits DM2, which is the same Semrock long-pass DM used in the CRM1. This reflects light below $790 \mathrm{~nm}$, and so the laser light is directed down and through the 60x Olympus water immersion objective and onto the sample. Both Rayleigh and Raman scattered light travel back through the microscope objective, and the latter passes through DM2, while the former is reflected. Two more edge filters designed to filter light below $790 \mathrm{~nm}$, for $\mathrm{EF} 1$, and below $785 \mathrm{~nm}$, for EF2, further remove any laser light that may have made it through DM2. The Raman scattered light is then focused by L2, a $50 \mathrm{~mm}$ focal length biconvex lens, onto the spectrometer input fiber. 
The brightfield light path is as follows: light is emitted by a $2350 \mathrm{~mW}, 700 \mathrm{~mA}$ ThorLabs $6500 \mathrm{~K}$ white light LED (MCWHLP1). The LED uses a single colour diode which is phosphor coated to attain a full spectrum. The emitted light is collected by a 2-inch diameter, $32 \mathrm{~mm}$ focal length plan-convex lens, L5. This light is then transmitted through a cage cube that can have a mirror in it if transmitted illumination is desired, or left empty if epi-illumination is desired. The trans-illumination light path has the LED light reflected off the mirror in the cube forward (out-of-page) to a mirror below the stage which reflects the light upwards and through a condenser lens, L6, which focuses it onto the sample.

In the epi-illumination path, the LED light continues past the cube and is then directed forward (out-of-page) and upward by two turning mirrors. It then passes through a 1-inch diameter $200 \mathrm{~mm}$ focal length spherical lens, L4, which focuses an image of the LED diode onto the top pupil of the microscope objective. The reason for this is explained in section 6.2.3. Before reaching the objective, it passes through a beamsplitter, which reflects $20 \%$ of the light rightward, after which a mirror reflects it onto DM2, which reflects it onto DM1, which reflects it onto into the objective. The objective then acts as the condenser, focusing the LED light onto the sample.

For both epi-illuminiation, where the LED light is reflected off of the sample, and trans-illumination, where it is transmitted through the sample, the light forming brightfield image is collected by the microscope objective, reflected off of DM2 followed by DM1 and then the turning mirror below it, through the beamsplitter, with $80 \%$ efficiency, then off of a final turning mirror after which it is focused by 1-inch, $100 \mathrm{~mm}$ focal length spherical lens, L3, onto the brightfield camera. 


\subsubsection{High power laser}

Perhaps the most substantial change to the new system has been the addition of a new, higher power laser. This laser, an Ondax BT-785-PLR500 (Ondax, Monrovia, CA, USA), has a factory tested and lab verified maximum out-of-fiber power of $470 \mathrm{~mW}$ compared to $100 \mathrm{~mW} \max$ power for the old laser. Its line width was found to be $<0.06 \mathrm{~nm}$ in factory testing. It is fiber coupled and the coupling fiber is a $2 \mathrm{~m}$ long multimode fiber with a $105 \mathrm{~nm}$ diameter core and $0.22 \mathrm{NA}$.

\subsubsection{Fiber coupling}

Both the laser and the spectrometer were fiber coupled in the new setup. This increases the modularity of our setup and simplifies it by reducing the number of mirrors and lenses required. Thus, realignment can be performed much more quickly than before.

The previous setup had 9 turning mirrors between the laser and spectrometer, one dichroic mirror, four lenses in translation cage plates - allowing for $\mathrm{x}-\mathrm{y}$ translation relative to laser path along $\mathrm{z}$ - and a pinhole in a cage plate as well. All of these elements, apart from the dichroic, had two knobs that had to be fine tuned during alignment. The dichroic mirror sat in a cage cube and could be rotated to achieve a $45^{\circ}$ angle relative to the laser and signal optical paths.

The fiber coupling reduces the number of turning mirrors in the new setup to five, four of which are for the brightfield imaging only, and two of those are simply for guiding light from the illumination source, and are much less sensitive to minor drifts in alignment. Thus, only one turning mirror is currently in the laser/signal path of the apparatus. The number of dichroics has increased to two, with one being used for brightfield imaging in order to replace the mirror that had to be manually 


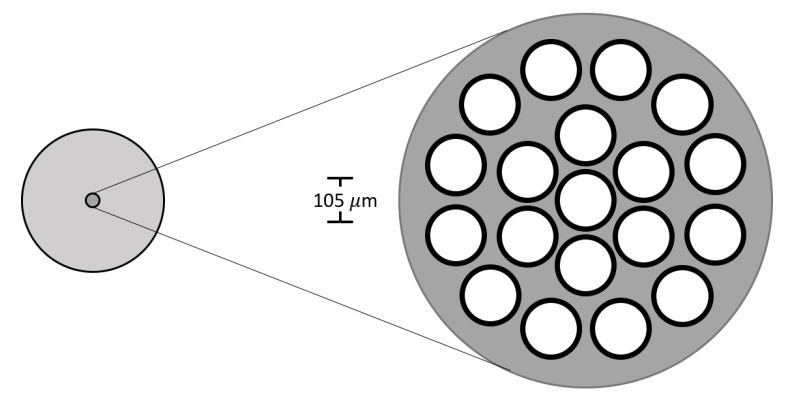

Figure 6.2: fiber core layout for input end of bundled optical fiber.

inserted before. The number of lenses between the laser and the spectrometer, ignoring the microscope objective, has been reduced to two: one for collimating the laser light leaving the laser coupled fiber, and one for focusing the Raman signal into the spectrometer coupled fiber bundle. The total number of optical components that require manual tuning during alignment has thus been substantially reduced.

The fiber that couples to the spectrometer is a custom-built bundled fiber (FiberTech Optica, Kitchener, ON, Canada) consisting of 19 fibers of $105 \mu \mathrm{m}$ each. On one end of the fiber, these are arranged to cover a circular area with a core of 7 fibers in a hexogonal lattice with a surrounding ring of 12 outer fibers. This layout is shown in Figure 6.2.

Since the incoming Raman light will generally have a Gaussian intensity profile, a majority of the light enters through the inner 7 fibers, assuming the light path is properly centred. The other end of the fiber has the 19 fibers spread out linearly in a line. This is the end that couples to the spectrometer. Having the light come out of the fiber in a vertical line is desirable since the entrance to the spectrometer is a vertical slit. Furthermore, since the CCD is currently being used in a column scanning mode, it is beneficial to spread the light out vertically over as many pixels in a column as possible, in order to reduce the chance of saturating any individual pixel. Thus, having a line output entering the spectrometer allows for a large vertical distribution of light on the CCD without losing light due to the slit. An image of line 


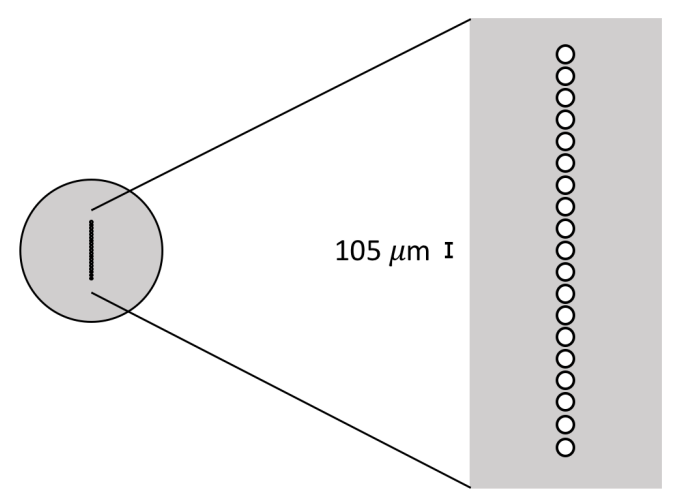

Figure 6.3: fiber core layout for output end of bundled optical fiber.

output fiber from the CCD itself is given in Figure 6.3

\subsubsection{Improved brightfield imaging}

Apart from replacing most of the laser path and signal path with optical fibers, the largest structural changes done to the apparatus are related to the brightfield imaging system. In order to eliminated the necessity of manually inserting and removing a mirror when switching between brightfield imaging and data acquisition modes, the new setup uses a second long pass dichroic, DM1, which has been placed in front of the main long pass dichroic. Rather than reflecting the laser down to the microscope objective, this dichroic lets it pass, only reflecting light at $750 \mathrm{~nm}$ or shorter wavelengths. The new LED used for illumination is a white light source covering a roughly 450-700 $\mathrm{nm}$ spectrum.

Since the sample is now illuminated with light that is reflected by both dichroics, the brightfield image no longer passes through the DM2 along with the signal as it did before, but now is reflected by the the first dichroic above the microscope objective, and then by the second one, downward. Two turning mirrors and lens guide the light to the camera. Thus, brightfield imaging does not require any mechanical changes be made to the apparatus between measurements, requiring only the LED power switch 
be flipped.

The new setup also allows for either epi-illumination or transmitted illumination of the sample, by placement of a single mirror. When in place, this mirror reflects the LED light below the stage, where a turning mirror then reflects it upwards and a lens focuses it onto the sample, providing transmissive illumination. For opaque samples, illumination must be reflective, and so the mirror is removed and the light travels a somewhat longer path until reaching the microscope objective which focuses the light onto the sample. This epi-illumination path requires a beam splitter to reflect the LED light but allow the reflected light to reach the camera. This path was created such that it satisfies the requirements of Kohler illumination, meaning the LED plane (object plane) is completely defocused on the sample (image plane), and thus the light intensity is uniform over the sample, improving image quality. This is done by bringing the image of the LED into focus at the back focal plan of the microscope objective. ${ }^{55}$

\subsection{Performance}

The new system is capable of reaching $(145 \pm 2) \mathrm{mW}$ power at the sample. It has an approximately $8 \mu \mathrm{m}$ spot size, thus the maximum intensity at the sample is approximately $3 \times 10^{5} \mathrm{~W} / \mathrm{cm}^{2}$. Spot diameter was determined by getting an image scale reference from a slide with a $10 \mu \mathrm{m}$ scale engraved on it. This was used to get a $\mu \mathrm{m} /$ pixel value in Image J, and then using a brightfield image of the laser spot to measure its diameter. A more rigorous examination of laser spot size and its intensity distribution will require a laser profiler.

The depth of the focal volume was determined to be approximately $30 \mu \mathrm{m}$. The method used was by taking spectra in $1 \mu \mathrm{m}$ increments in the axial direction of a 


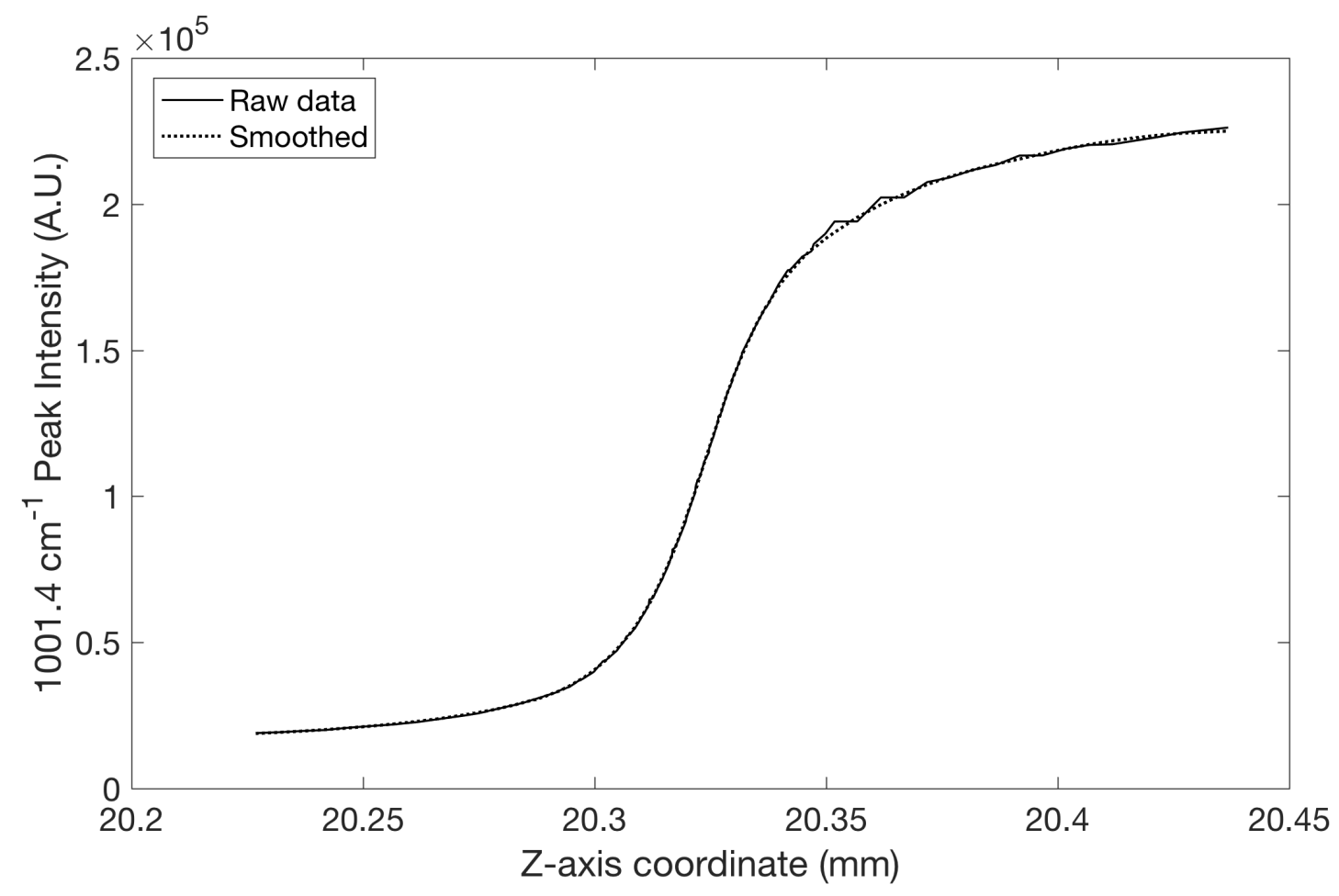

Figure 6.4: Peak intensity at $1001.4 \mathrm{~cm}^{-1}$ for polystyrene vs depth along z-axis.

transparent polystyrene dish, beginning above the sample surface where there is no signal, and then slowly entering into it. By recording the peak intensity of the main polystyrene peak at $1001.4 \mathrm{~cm}^{-1}$, a set of values are found that are proportional to the laser power in volume overlapping the sample and the objective's focal volume. The resulting data provides a rough estimate of the cumulative distribution of the actual axial power distribution of the beam in the focal volume. ${ }^{73,74}$ This cumulative laser power distribution is shown in Figure 6.4.

Taking the derivative of this, by taking the slope between each pair of adjacent data points, produces a new set of derived data points. Note that it was necessary to apply a Savitzky-Golay filter to the original data before taking the derivative, in order to reduce the amount of jaggedness in the result. Finding a smooth fit through these derived points (e.g. fitting a Lorentzian or Voigt profile to them), or using a smoothing filter, then provides a peaked distribution from which FWHM can be 


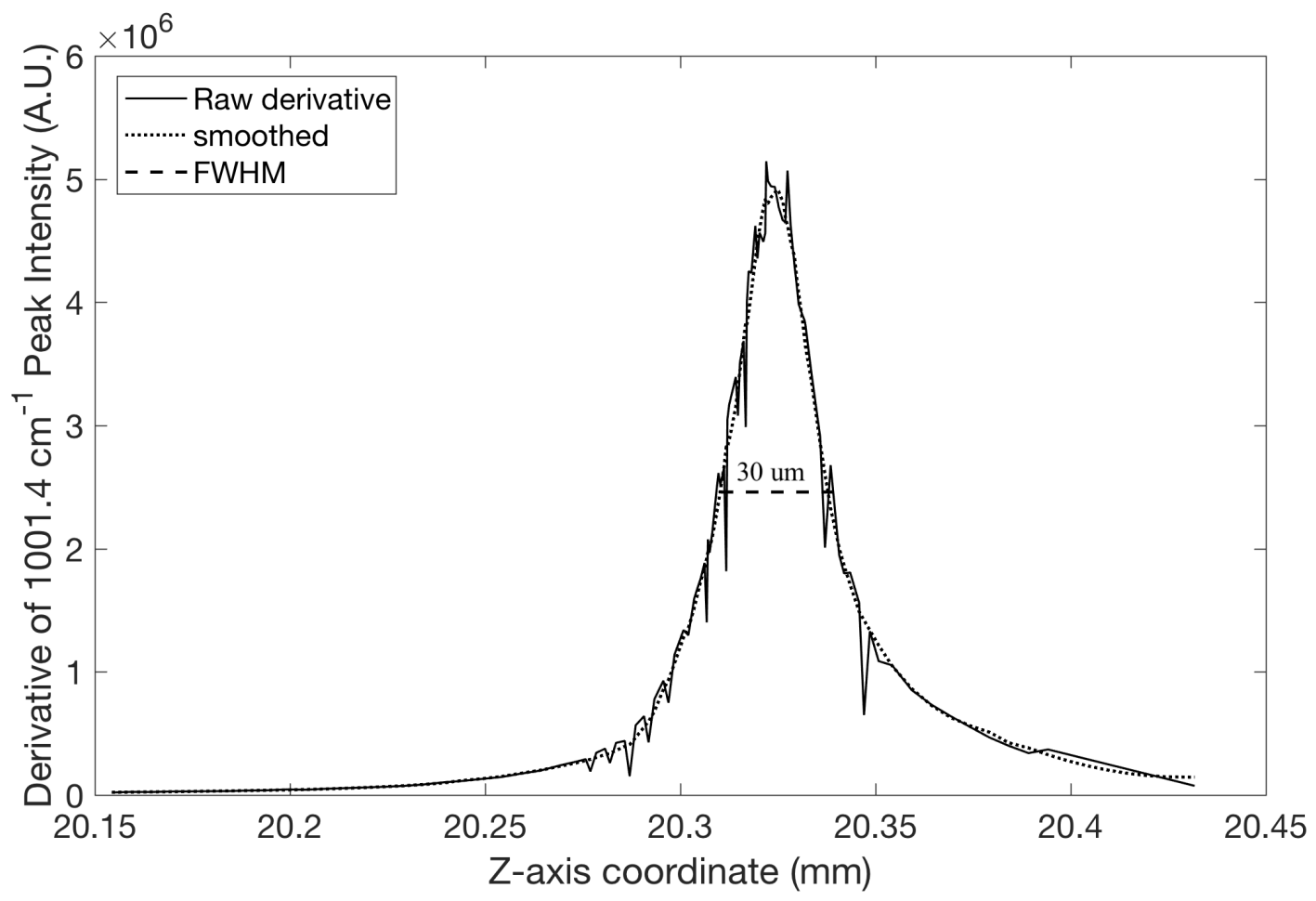

Figure 6.5: Plot of axial distribution of laser power in focal volume. FWHM, at approximately $30 \mu \mathrm{m}$, can be considered the axial resolution of the new instrument.

determined, and thus an estimate of the axial resolution of the instrument. In this case, a Savitzky-Golay filter was used, hence, this filter was used before and after taking the derivative of the z-depth peak intensity data.

\subsubsection{Silicon and polystyrene}

The main Raman peaks for both silicon and polystyrene showed a tremendous increase in peak intensity when normalizing for laser power. Whereas the previous CRM was capable of reaching around $32 \mathrm{k}$ counts/s for the Si peak at $520 \mathrm{~cm}^{-1}$, new system is capable of reaching over $68 \mathrm{k}$ counts/s at $30 \%$ laser power $(45 \mathrm{~mW}$ power at sample). A plot of the silicon spectra for both CRM1 and CRM2 is given in Figure 6.6. Note that there are two CRM2 spectra, one dashed and one dotted. The dashed spectrum is the raw silicon spectrum with the laser at 30\% power, while the dotted spectrum 


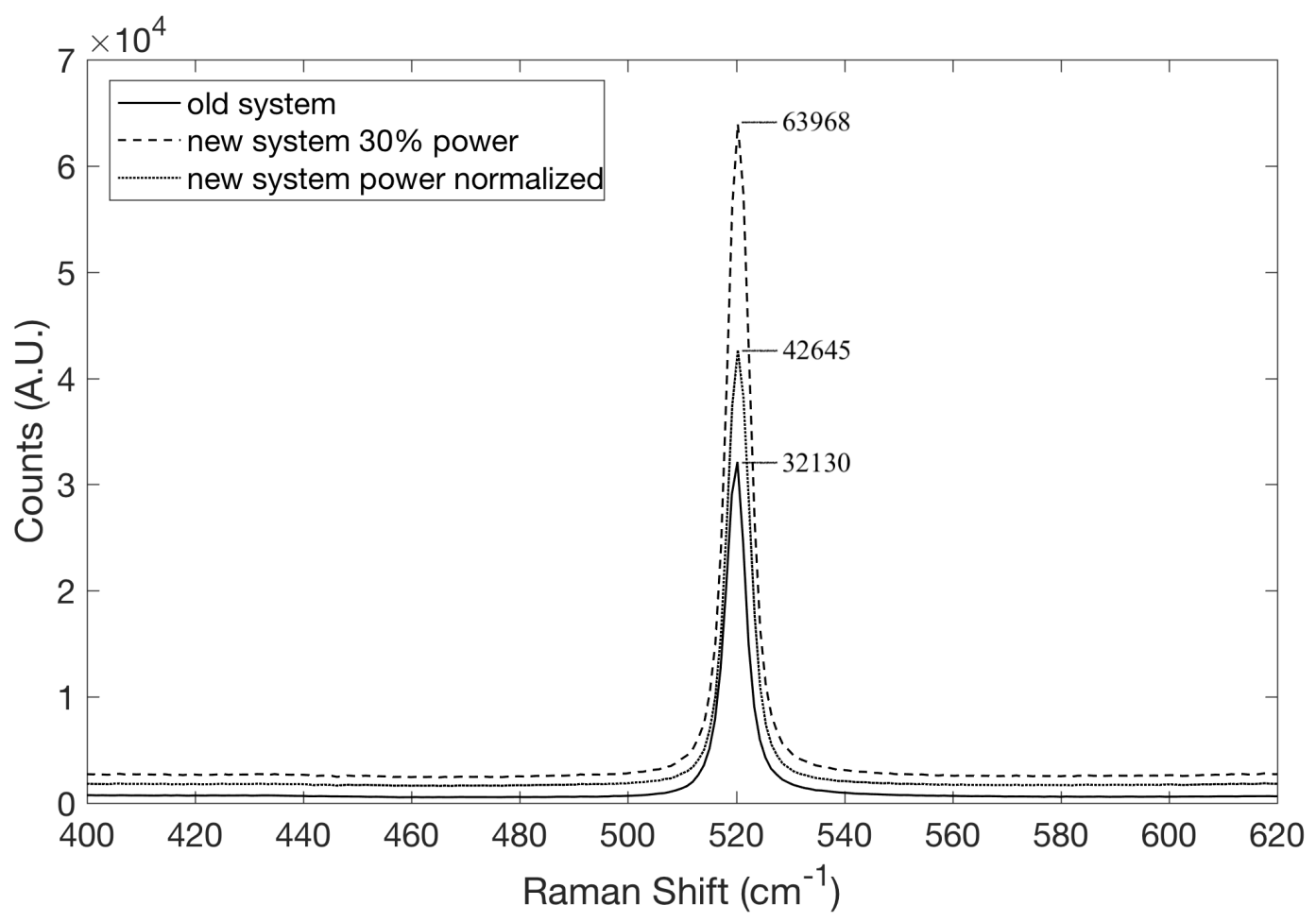

Figure 6.6: Silicon spectra for CRM1 and CRM2. Peak heights for the peak at $520 \mathrm{~cm}^{-1}$ are given.

is the same spectrum after normalizing for power at sample, that is, multiplying by a factor of 30/45. This was done to demonstrate signal efficiency for same power at sample.

An even larger improvement was seen with polystyrene. The largest peak in polystyrene, at $1001.4 \mathrm{~cm}^{-1}$ showed a peak intensity of $26 \mathrm{k}$ counts/s, compared to just $2 \mathrm{k}$ counts $/ \mathrm{s}$ in the previous setup. This is primarily due to the polystyrene being transparent and the non-confocal nature of the new setup means it has a much deeper focal volume and can sample Raman scattered light from a much larger volume. The spectra showing this are given in Figure 6.7. As before, there are two spectra corresponding to the new setup, one representing the raw output at $40 \%$ power, and the other normalized for power at sample to demonstrate signal efficiency. 


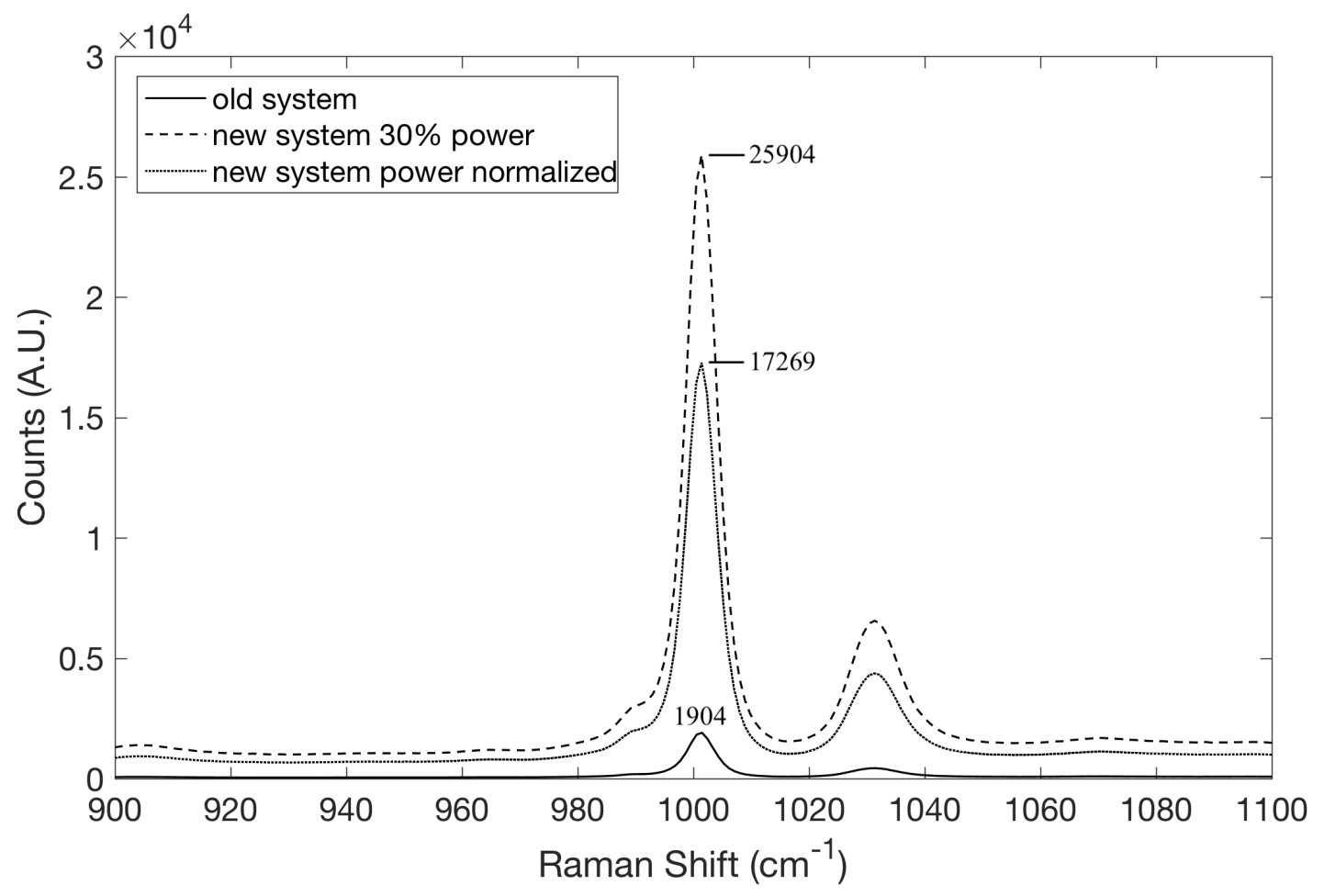

Figure 6.7: Polystyrene spectra for CRM1 and CRM2. Peak heights for the peak at $1001.4 \mathrm{~cm}^{-1}$ are given. 


\section{Chapter 7}

\section{Conclusions and future work}

\subsection{Conclusions}

This work has demonstrated the use of Raman spectroscopy as a viable tool for investigating the differences in HLE cells exposed to a varying, low-dose range of ionizing radiation in vitro. It has shown that it is possible to produce Raman spectra from single cells using a $1 \mu \mathrm{m}$ spot size, confocal Raman microscope that are viable for this purpose by averaging over multiple point spectra within the cell. Statistically significant, visible differences between the mean spectra of each dose and control have been shown, and multivariate statistical classification techniques (PCA-LDA) have been used to discriminate between spectra of cells exposed to a certain dose and a spectra from a control set to $a \geq 74 \%$ accuracy. This demonstrates the possible viability of using RS as a diagnostic tool in cases of low-dose radiation exposure to the lens of the eye. Use of the discriminant vectors to determine which changes in Raman peak intensity correspond to dose provided a list of biomolecules that show variability between dose and control sets. This has given insight into some of the possible biological mechanisms induced by low-dose exposures and may prove useful 
to future research into cataract formation. Fitting an induced-repair model survival curve to some of the Raman peaks as a function of dose, as well as to clonogenic assay data from Health Canada, has demonstrated that such repair processes may be at play in HLE cells as well.

The limiting factors of using the original version of the Raman microscope (CMR1) have been discussed, namely the limits on throughput and spectral generality, which are inevitable with a diffraction limited laser spot and a laser power limit of $30 \mathrm{~mW}$ at sample. A new version, CRM2, has been built and shown to allow for up to $145 \mathrm{~mW}$ laser power at the sample and an 8 times larger spot diameter. The laser spot volumetric coverage has been increased by a factor of 6 , further increasing the sampling volume, but at the cost of removing confocality. Results from silicon and polystyrene samples show an increase in signal per second of 1.32 and 9 times respectively, and thus suggest quicker acquisition times will be possible. Combined with a brightfield system that no longer requires manual placement and rotation of a mirror, this new setup should allow for more spectra to be acquired per batch, and thus increase the robustness of our statistical analyses. The fact that the larger spot size will provide a spectrum more representative of the entire cell will also help reduce intercell variability in spectra resulting from variability in intracellular spectra. This too will improve results and allow for higher discriminatory accuracies as it is removed as a confounding factor.

\subsection{Future work}

As discussed in the introduction, the long term goal of this work is to determine whether Raman spectroscopy is suitable for purposes of in vivo radiation dosimetry of the lens of the eye and/or detecting the precursors to radiation induced cataract formation. This requires more extensive determination of the sensitivity of RS at low 
doses for blind sample sets. Inter-batch variability means that Raman spectra from cells from a large number of batches will be required, and this will require a high throughput device. Now that this device has been built, necessary next steps are tuning it to maximize performance with biological samples and developing methods and protocols for acquiring Raman spectra from HLE cells in particular. Some of this work has begun with measurements being made on live and fixed HLE cells in pelleted form. This work is too recent to be covered in this thesis, but so far quartz still seems to be an ideal substrate, even if it is out of the focal volume being analyzed, due to its transparency and small amount of fluorescence. Reflective samples such as gold and aluminum were found to have much higher background; the reasons why are yet to be determined.

On top of being able to discriminate between dose and control from a blind sample set, it would be nice to be able to distinguish between doses as well. This also will require a large increase in data collection throughput as classification accuracy decreases as the number of classes increases for the sample sample size. The sample size of this work was insufficient for successful multi-class discrimination. Using more advanced classification methods than PCA-LDA will likely be necessary as well, since the induced repair model suggests a non-monotonic response among many peaks. This may make multi-class datasets not linearly separable. Classification using artificial neural networks (ANNs), decision trees, and support vector machines (SVMs) with non-linear kernels should thus be used on future data sets as well, but care must be taken as these more complex models can more easily fit a training set and lead to poor test results. ${ }^{75}$ Deriving which features are most important to a given discrimination from these techniques is also more complex since they are not simple projections as in the case of PCA-LDA.

Another future improvement to current methods that can be made is a method for determining which biomolecule, or combination of biomolecules, are responsible 
for a given Raman peak to some probability, and to what proportion. This will allow for determining the certainty of our molecular assignment results quantitatively, and will reduce the chance of making incorrect assumptions that lead to unfruitful future investigations. Methods such as spectral decomposition into known molecular spectra have been demonstrated in various works, ${ }^{76}$ and may prove useful. Matching principal components to such spectra could be another method, but the literature suggests that independent component analysis (ICA) provides components that are more likely to match constituent spectra than PCA. ${ }^{77}$ Thus, using ICA on future datasets and testing its usefulness will also prove useful.

Finally, developing the ability to acquire useful spectra in vivo will necessarily involve acquiring Raman spectra from live cells. Our lab has an incubator built into the stage that can be uses for live samples in vitro, and using as such will be a necessary step towards the final goal. Experiments on live cells will require much more careful experimental design, as the cells will perform their biological functions, such as going through the cell cycle, while measurements are being taken. Being able to acquire spectra quickly will thus be a necessity to allow for snapshots of a cell's biomolecular state over time. Being able to to distinguish radiation induced changes from all the other factors that a live cell brings will be a challenge but it is a necessary step if Raman spectroscopy is to prove itself useful for radiobiological diagnostic purposes. 


\section{Appendix A}

\section{Derivations}

\section{A.1 Normal Coordinates}

This derivation is primarily based on material in Molecular Vibrations by Wilson,

Decius, and Cross. ${ }^{49}$ Consider a molecule with $N$ atoms. For the $i^{\text {th }}$ atom, with equilibrium position, in Cartesian coordinates, of

$$
\mathbf{x}_{i 0}=x_{i 0} \hat{i}+y_{i 0} \hat{j}+z_{i 0} \hat{k}
$$

The displacement of this atom from its equilibrium position, in Cartesian coordinates, at time $t$, is then

$$
\begin{aligned}
\mathbf{x}_{i}^{\prime}(t) & =x_{i}^{\prime}(t) \hat{i}+y_{i}^{\prime}(t) \hat{j}+z_{i}^{\prime}(t) \hat{k} \\
& =\left(x_{i}(t)-x_{i 0}\right) \hat{i}+\left(y_{i}(t)-y_{i 0}\right) \hat{j}+\left(z_{i}(t)-z_{i 0}\right) \hat{k} .
\end{aligned}
$$


The kinetic energy of this system in classical mechanics is given by

$$
\begin{aligned}
T & =\frac{1}{2} \sum_{i=1}^{N} m_{i}\left(\frac{d \mathbf{x}_{i}^{\prime}}{d t}\right)^{2} \\
& =\frac{1}{2} \sum_{i=1}^{N} m_{i}\left[\left(\frac{d x_{i}^{\prime}}{d t}\right)^{2}+\left(\frac{d y_{i}^{\prime}}{d t}\right)^{2}+\left(\frac{d z_{i}^{\prime}}{d t}\right)^{2}\right]
\end{aligned}
$$

We can choose mass-weight displacement coordinates to eliminate the mass factor, by choosing

$$
\begin{aligned}
& q_{1}=\sqrt{m_{1}} x_{1}^{\prime} \\
& q_{2}=\sqrt{m_{1}} y_{1}^{\prime} \\
& q_{3}=\sqrt{m_{1}} z_{1}^{\prime} \\
& q_{4}=\sqrt{m_{2}} x_{2}^{\prime} \\
& \vdots \\
& q_{3 N}=\sqrt{m_{N}} z_{N}^{\prime},
\end{aligned}
$$

and so Equation A.3 can be written as

$$
T=\frac{1}{2} \sum_{i=1}^{3 N}\left(\frac{d q_{i}}{d t}\right)^{2}
$$

We can then represent the potential energy, $\mathrm{V}$, of a system with small displacements using a Taylor expansion about the equilibrium position of each $q_{i}$,

$$
V=V_{0}+\sum_{i=1}^{3 N}\left(\frac{\partial V}{\partial q_{i}}\right)_{0} q_{i}+\frac{1}{2} \sum_{i, j}^{3 N}\left(\frac{\partial^{2} V}{\partial q_{i} \partial q_{j}}\right)_{0} q_{i} q_{j}+\ldots
$$

To simplify things, we can define minimum potential energy, which is the equilibrium potential energy $V_{0}$, to be zero. Then, applying the harmonic approximation by ignoring terms of order greater than 2, which is valid for small displacements, and 
noting that the first derivative of $\mathrm{V}$ for each $q_{i}$ will be 0 at equilibrium, because the equilibrium position corresponds to minimum potential energy, meaning the second term is 0 , gives an approximate form of $V$,

$$
\begin{aligned}
V & =\frac{1}{2} \sum_{i, j}^{3 N}\left(\frac{\partial^{2} V}{\partial q_{i} \partial q_{j}}\right)_{0} q_{i} q_{j} \\
& =\frac{1}{2} \sum_{i, j}^{3 N} F_{i j} q_{i} q_{j}
\end{aligned}
$$

where the $3 N \times 3 N$ matrix $\mathbf{F}$, given by elements

$$
(\mathbf{F})_{i j}=F_{i j}=\left(\frac{\partial^{2} V}{\partial q_{i} \partial q_{j}}\right)_{0}
$$

is called the mass-weighted Hessian matrix. We can then transform our coordinate system into one which diagonalizes $\mathbf{F}$, and thus allows us to write $\hat{V}$ in terms of a sum of linearly independent harmonic oscillators. These new coordinates are called the normal coordinates of the system, and are given by

$$
Q_{i}=\sum_{j=1}^{3 N} L_{j i} q_{i}
$$

where $L_{j i}$ is an element of the matrix $\mathbf{L}$ which diagonalizes $\mathbf{F}$, that is

$$
\mathbf{L F}=\mathbf{L D}
$$

where $\mathbf{D}$ is a diagonal matrix . The columns of $\mathbf{L}$ are therefore eigenvectors of $\mathbf{F}$, and the diagonal elements of $\mathbf{D}$ the eigenvalues. 


\section{A.2 Mean and variance of $L D$ scores}

For a data matrix $\mathbf{X}$, with mean row vector $\overline{\mathbf{X}}$, the projection of the mean onto the line passing through unit vector $\mathbf{a}$ is equal to the mean of projected data set:

$$
\begin{aligned}
\overline{\mathbf{X}} \mathbf{a} & =\frac{1}{N}\left(\mathbf{x}_{1}+\mathbf{x}_{2}+\ldots+\mathbf{x}_{N}\right) \mathbf{a} \\
& =\frac{1}{N}\left(\mathbf{x}_{1} \mathbf{a}+\mathbf{x}_{2} \mathbf{a}+\ldots+\mathbf{x}_{N} \mathbf{a}\right) \\
& =\overline{\mathbf{X}} \mathbf{}
\end{aligned}
$$

For a covariance matrix $\mathbf{C}$,

$$
\begin{aligned}
\mathbf{a}^{\dagger} \mathbf{C a} & =\mathbf{a}^{\dagger}\left[\frac{1}{N-1} \sum_{i=1}^{N}\left(\mathbf{x}_{\mathbf{i}}-\overline{\mathbf{X}}\right)^{\dagger}\left(\mathbf{x}_{\mathbf{i}}-\overline{\mathbf{X}}\right)\right] \mathbf{a} \\
& =\frac{1}{N-1} \sum_{i=1}^{N} \mathbf{a}^{\dagger}\left(\mathbf{x}_{\mathbf{i}}^{\dagger}-\overline{\mathbf{X}}^{\dagger}\right)\left(\mathbf{x}_{\mathbf{i}}-\overline{\mathbf{X}}\right) \mathbf{a} \\
& =\frac{1}{N-1} \sum_{i=1}^{N}\left(\mathbf{a}^{\dagger} \mathbf{x}_{\mathbf{i}}^{\dagger}-\mathbf{a}^{\dagger} \overline{\mathbf{X}}^{\dagger}\right)\left(\mathbf{x}_{\mathbf{i}} \mathbf{a}-\overline{\mathbf{X}} \mathbf{a}\right) \\
& =\frac{1}{N-1} \sum_{i=1}^{N}\left(\mathbf{x}_{\mathbf{i}} \mathbf{a}-\overline{\mathbf{X}} \mathbf{a}\right)^{\dagger}\left(\mathbf{x}_{\mathbf{i}} \mathbf{a}-\overline{\mathbf{X}} \mathbf{a}\right) \\
& =\frac{1}{N-1} \sum_{i=1}^{N}\left(s_{i}-\overline{\mathbf{s}}\right)^{2}
\end{aligned}
$$

which is simply the variance the scores, that is the values of the data that made $\mathbf{C}$ projected onto a. There can be no covariance for scores since they are one dimensional.

\section{A.3 Variance of LOOCV accuracy}

The accuracy of LOOCV is the mean value of each classified data point. Each point is either classified correctly or not, and so has accuracy 1 or 0 . This is a random variable due to the data points being random variables, and so we can find the sample mean, 
$A$, which we report as the LOOCV accuracy. Because each point is either 1 or 0 , we can find an expression for the error of $A$, its sample standard deviation of the mean, in terms of itself and $N$, the number of samples in the data set. First, we find the sample variance of the population of accuracies:

$$
\begin{aligned}
\sigma^{2} & =\frac{1}{N-1}\left(\sum_{k=1}^{N A}(1-A)^{2}+\sum_{k=1}^{N(1-A)}(-A)^{2}\right) \\
& =\frac{1}{N-1}\left(N A(1-A)^{2}+N(1-A) A^{2}\right) \\
& =\frac{1}{N-1}\left(N A-2 N A^{2}+N A^{3}+N A^{2}-N A^{3}\right) \\
& =\frac{N A-N A^{2}}{N-1} \\
& =\frac{N A(1-A)}{N-1} \\
& =\frac{A(1-A)}{1-1 / N} .
\end{aligned}
$$

Note that in the above, the usual sum found in the expression for sample variance is split into two sums, one covering the case where a sample is classified correctly, and the other the cases when it is not. Now, the sample standard deviation is simply $\sigma$, and the sample standard deviation of the mean is $\sigma_{m}=\sigma / \sqrt{N}$. Hence, the error on $A$ for LOOCV need not be manually calculated, and is given simply by equation 3.34 (given again here),

$$
\sigma_{m}=\sqrt{\frac{A-A^{2}}{N-1}}
$$

\section{A.4 PCA using SVD}

The preferred algorithm for calculating principal components in the pricomp algorithm in $\mathrm{R}$ is via singular-value decomposition (SVD) of the mean centred data matrix. If the data matrix has been mean-centred, then the covariance matrix of the 
mean-centred data matrix, $\tilde{\mathbf{X}}$, is given by equation $3.24\left(C=\tilde{\mathbf{X}}^{\dagger} \tilde{\mathbf{X}} /(N-1)\right)$.

Now, performing a SVD on an $N$ by $m$ matrix (in this case the mean-centred data matrix) $\tilde{\mathbf{X}}$, means finding matrices $\mathbf{U}$ and $\mathbf{V}$ such that

$$
\tilde{\mathbf{X}}=\mathbf{U S V}^{\dagger}
$$

where $\mathbf{U}$ and $\mathbf{V}$ are unitary matrices and $\mathbf{S}$ is a diagonal matrix of singular values. Recalling equation 3.24, we have

$$
\begin{aligned}
\mathbf{V}^{\dagger} \mathbf{C V} & =\mathbf{V}^{\dagger} \frac{\tilde{\mathbf{X}}{ }^{\dagger} \tilde{\mathbf{X}}}{N-1} \mathbf{V} \\
& =\mathbf{V}^{\dagger} \mathbf{V} \frac{\mathbf{S}^{\dagger} \mathbf{U}^{\dagger} \mathbf{U S}}{N-1} \mathbf{V}^{\dagger} \mathbf{V} \\
& =\frac{\mathbf{S}^{2}}{N-1}
\end{aligned}
$$

From this equation, we see then that it is equivalent to Equation 3.27, with $\mathbf{D}=$ $\mathbf{S}^{2} /(N-1)$. It follows that $\mathbf{V}$ is the same in both equations, and its columns must be the principal components of $\tilde{\mathbf{X}}$. SVD is often used to find $\mathbf{V}$ to avoid the step of computing $\tilde{\mathbf{X}}^{\dagger} \tilde{\mathbf{X}}$. 


\section{Appendix B}

\section{Protocol for acquiring Raman spectra from HLE samples}

While collecting the spectra of the cells the following protocol has to be followed:

1) Before beginning the experiment, measure the spectra of a polystyrene dish to confirm if the alignment is correct. This involves turning on the laser and letting it run for 30 minutes, turning on the stage, and the spectrometer, which has to cool to -85 celsius. This cooling only begins once Andor SOLIS acquisition software has been started.

2) Take the sample of cells to be used for that day, place the quartz cover slip they are adhered to into the magnetic holder, add PBS, and place the sample with the holder onto the stage below the microscope objective.

3) Raise the stage until the objective lens just immerses in the solution. After that raise the stage using the fine-adjustment and continue raising it until the laser is focussed on the top surface of the quartz substrate.

4) Open the script in Andor SOLIS for recording nine 1-minute spectra, 3 frames 
averaged per point, which communicates with the stage automation script. Open a PowerShell (terminal) window in Windows and start the stage automation Python script. It will wait for you to press enter.

5) Find an area on the substrate with no cells adhered, focus the laser spot at the surface, and record a 5 minute background spectrum. This involves removing the neutral density filter from in front of the laser (there to avoid saturating brightfield CCD), removing the mirror for reflecting light to the brightfield CCD, turning off the illumination LED, and closing the curtain to darken the apparatus. The acquisition is not done with an Andor Basic script, it is just a 5 minute acquisition of five 1-minute frames. The save file requires '_bgd_' in the filename for data preprocessing.

6) Once the background is finished, open the curtain, place the ND filter back in front of the laser, and place the mirror back into the light path so that it goes to the CCD camera instead of the spectrometer. Move the stage along the $\mathrm{x}-\mathrm{y}$ axis to search for a cell to use, and write down the coordinates of the chosen cell.

7) Choose a spot in the nucleus that will be the top left corner of the $3 \times 3$ grid, and move the stage such that the laser spot is over that point. Record a brightfield image of the laser spot at this point.

8) Lower the stage by $5 \mu \mathrm{m}$ so that the laser focal volume is in the cell, remove the mirror, turn-off the back-illumination LED, remove the neutral density filter from in front of the laser, and pull the curtains.

9) In Andor SOLIS, hit the Run Script button. It will wait for the stage automation script to indicate it is ready, so press enter in the PowerShell window running the stage automation script. The script will ask for a file name with which to name the collected spectra. It is best to have a general one saved in the clipboard which can be pasted and a number added. Data from cells (i.e. not background) must have '_raw_' in the title for later post-processing. 
10) Once the spectra have been collected, turn back on the back-illumination LED, place the ND filter back in front of the laser, bring the cell into focus with the stage z-axis control, and record another brightfield image of the laser spot in the position which would be the bottom right corner of the $3 \times 3$ grid. Check to image from the top left corner to make sure a square between the two wouldn't fall outside the nucleus.

11) Repeat steps 6-10 four more times, making sure to check that the cell coordinates are different each time.

12) Repeat step 5, and then open the stage automation script in the PowerShill window that is used for cytoplasm. The only difference in the script is the points will be $1 \mu \mathrm{m}$ apart instead of $3 \mu \mathrm{m}$.

13) Repeat steps 6-11 for cytoplasm.

14) Repeat steps 5-13 three more times. You should now have 20 nucleus and 20 cytoplasm spectra.

15) Once the experiment is over, place the spectra, including background spectra, in the "SMIRF input" folder and run SMIRF in MATLAB. This SMIRF will background subtract the quartz fluorescence from the spectra and smooth them using a Savitzky-Golay filter.

16) Clean the microscope objective by rinsing in water and then ethanol. Put the sample back in the biosafety cabinet. Take a final spectrum of the polystyrene dish, then put it away, and shut down the laser, spectrometer, stage, back illumination LED, and any open programs. Turn off the "LASER ON" light. Turn out room lights and make sure doors are locked before leaving. 


\section{Appendix $\mathrm{C}$}

\section{Additional Figures}

\section{C.1 Cell cycle analysis results}

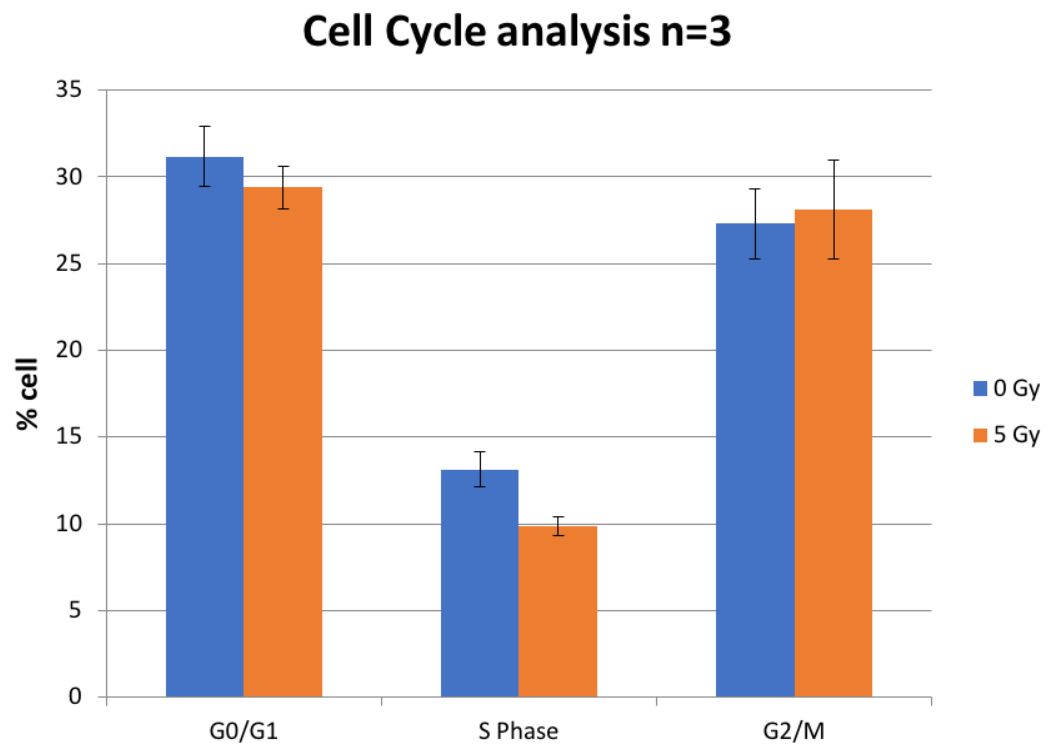

Figure C.1: Cell cycle percentages for 0 and 5 Gy for 24 hours post irradiation using Guava flow cytometry. 


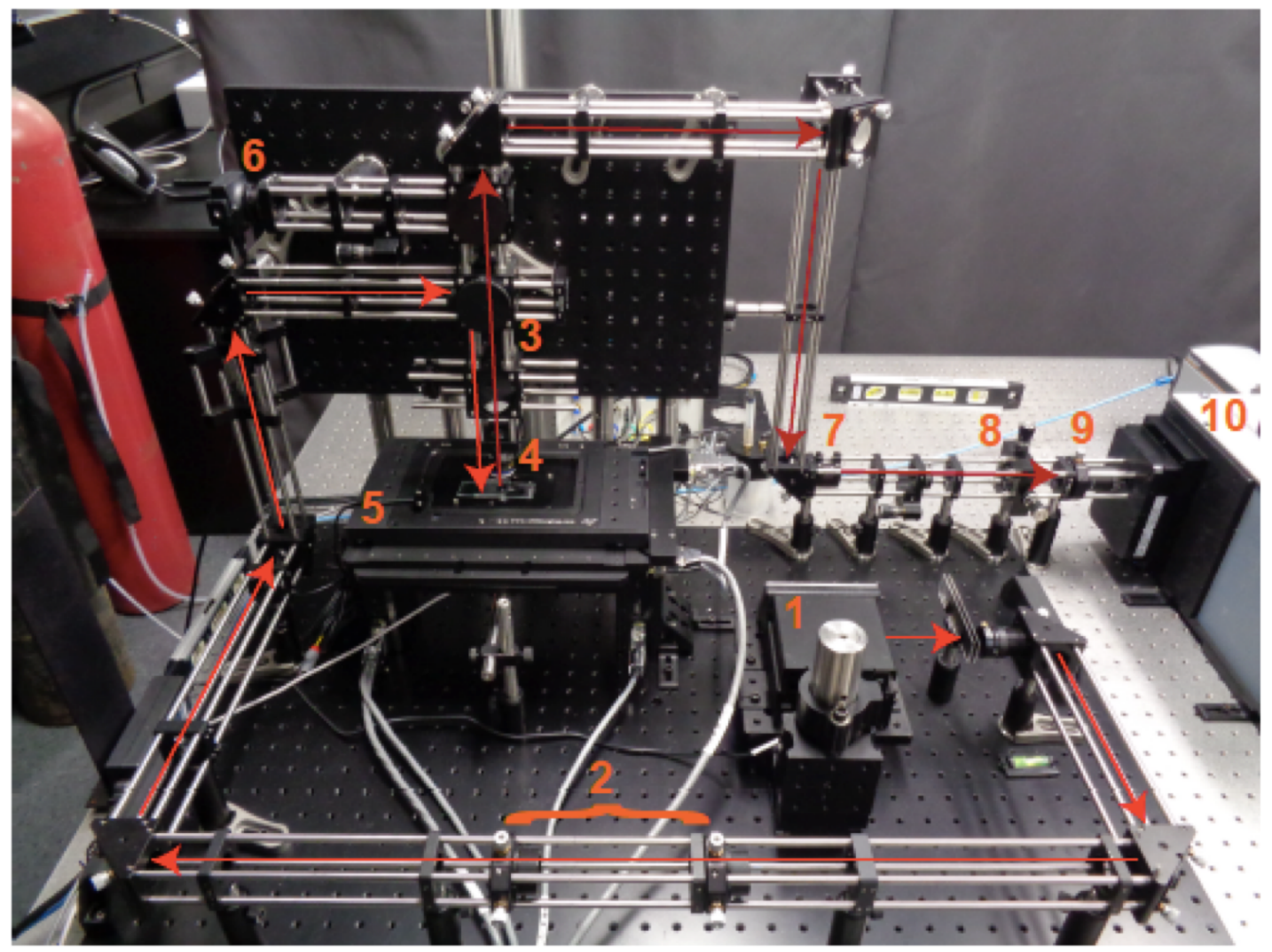

Figure C.2: Photograph of the the Carleton Raman Microscope used for this research. Components are as follows: 1 - laser, 2 - Beam expansion between two lenses, 3dichroic mirror, microscope objective above sample, 6 - brightfield camera, 7 - edge filters, 8 - pinhole, 9 - lens, 10 - spectrometer. 


\section{C.2 Photograph of Carleton Raman Microscope}

\section{versions 1 and 2}

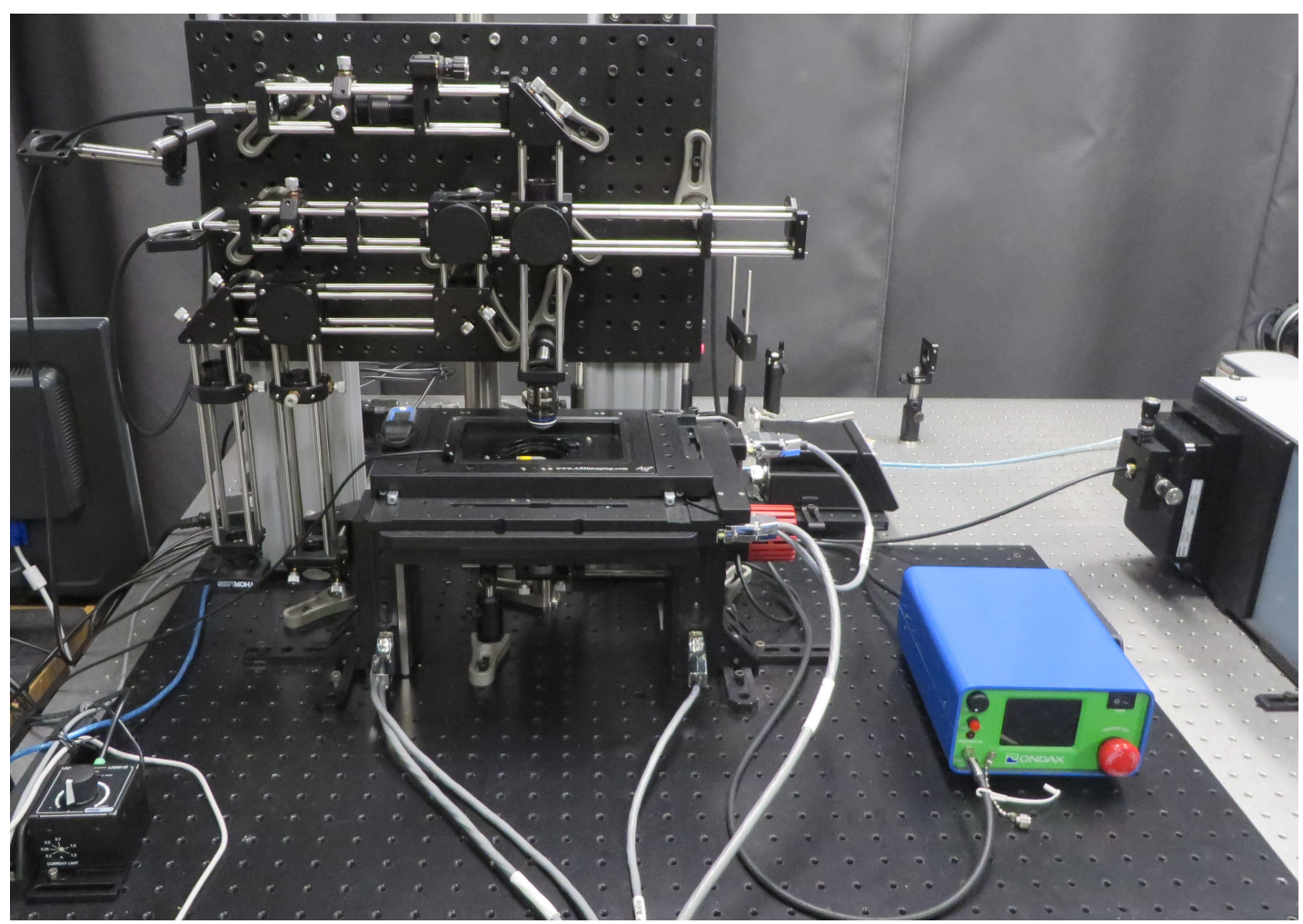

Figure C.3: Photograph of Carleton Raman Microscope version 2 (CRM2)

\section{C.3 Plots of accuracy vs number of PCs}

The plots of accuracy vs number of PCs which were used to determine the optimal number of PCs for each PCA-LDA performed are given here. 


\section{C.3.1 Nucleus}

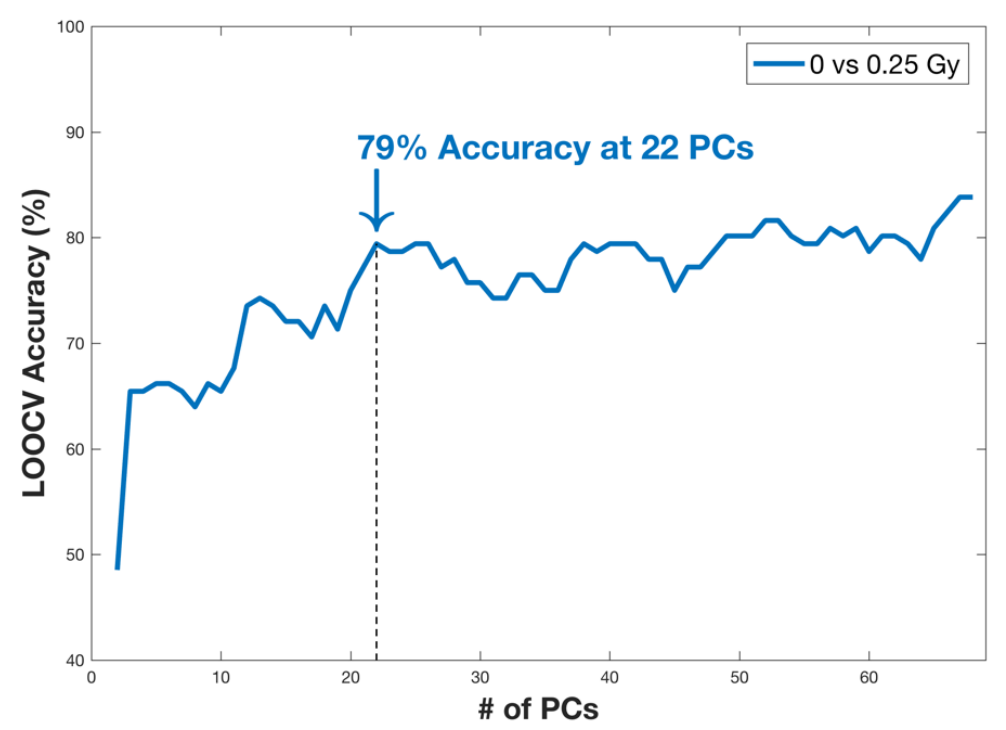

Figure C.4: Plot of LOOCV accuracy vs number of PCs used in the LDA for 0 vs 0.25 Gy, nucleus.

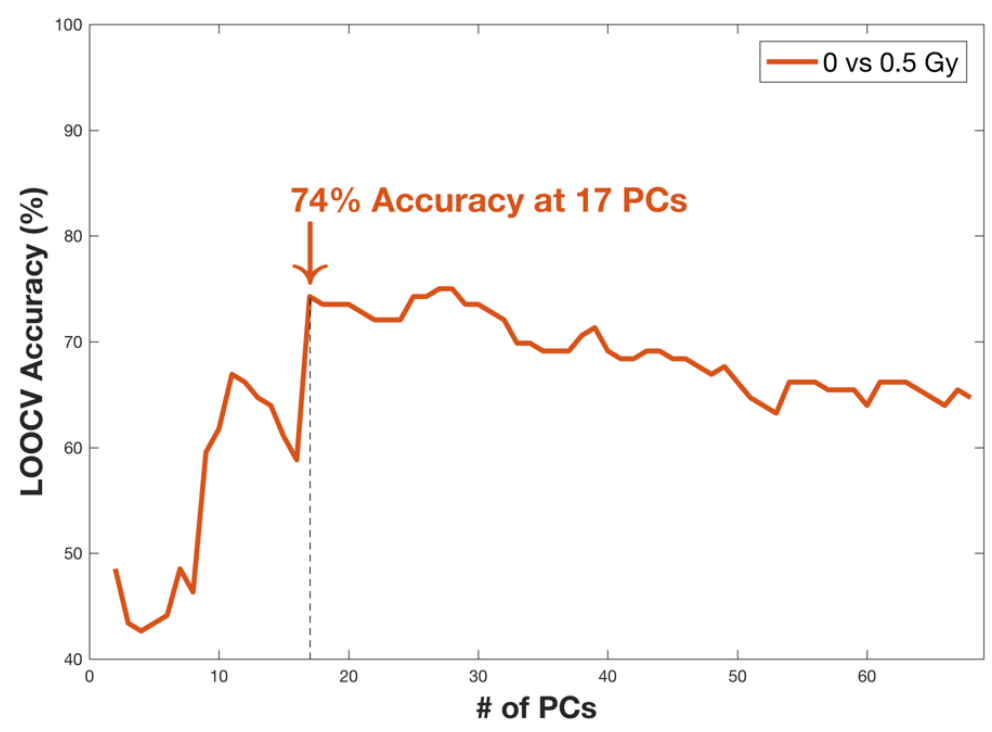

Figure C.5: Plot of LOOCV accuracy vs number of PCs used in the LDA for 0 vs 0.5 Gy, nucleus. 


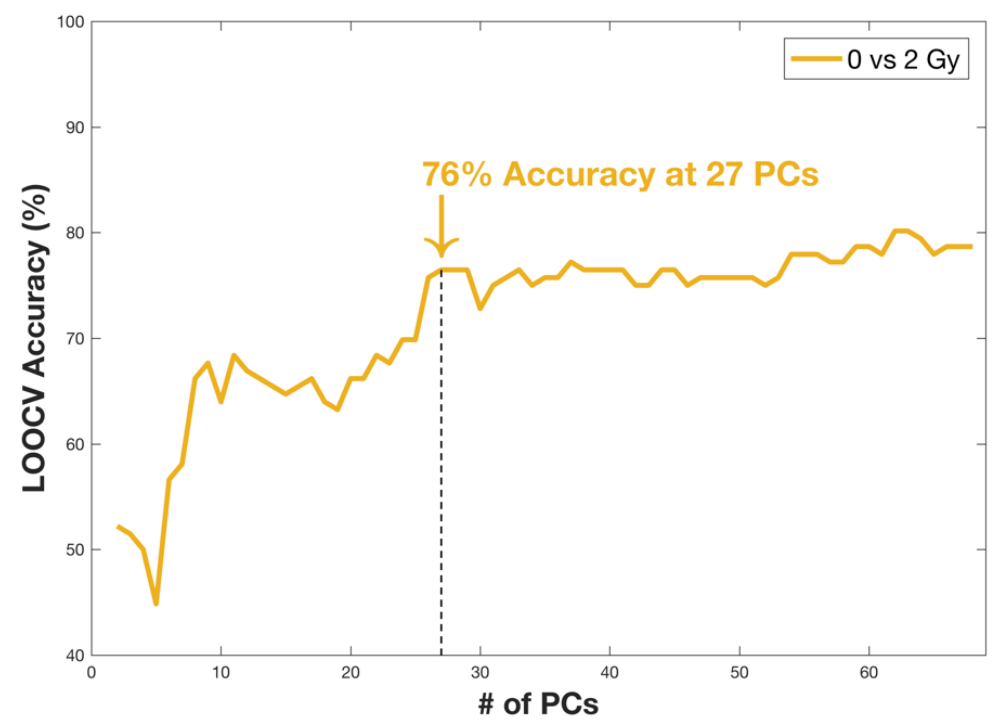

Figure C.6: Plot of LOOCV accuracy vs number of PCs used in the LDA for 0 vs 2 Gy, nucleus.

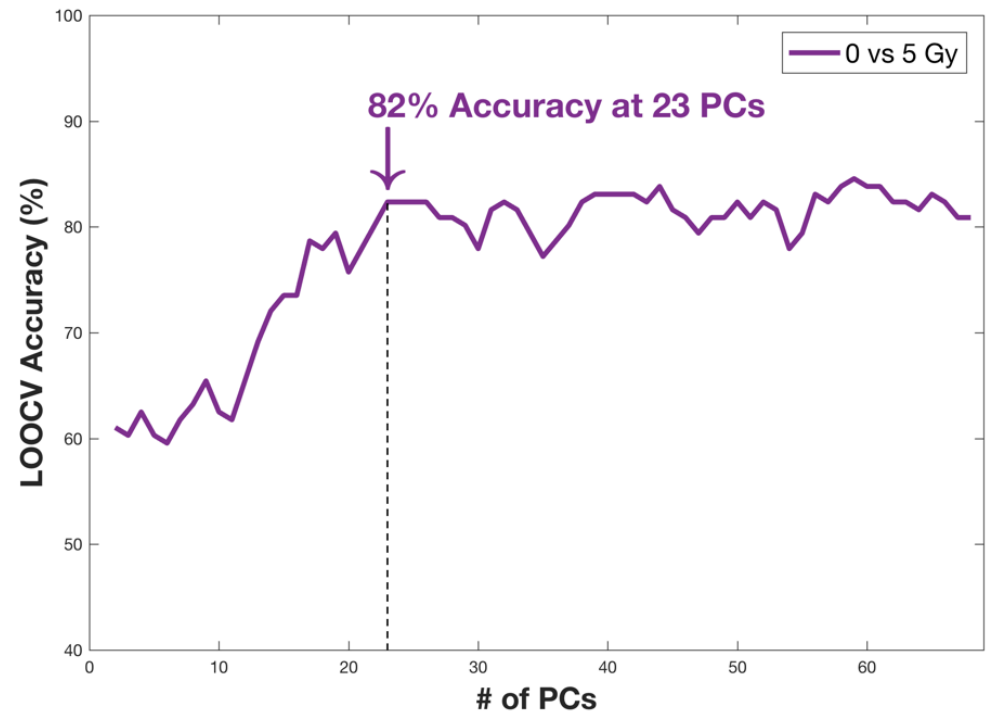

Figure C.7: Plot of LOOCV accuracy vs number of PCs used in the LDA for 0 vs 5 Gy, nucleus. 


\section{C.3.2 Cytoplasm}

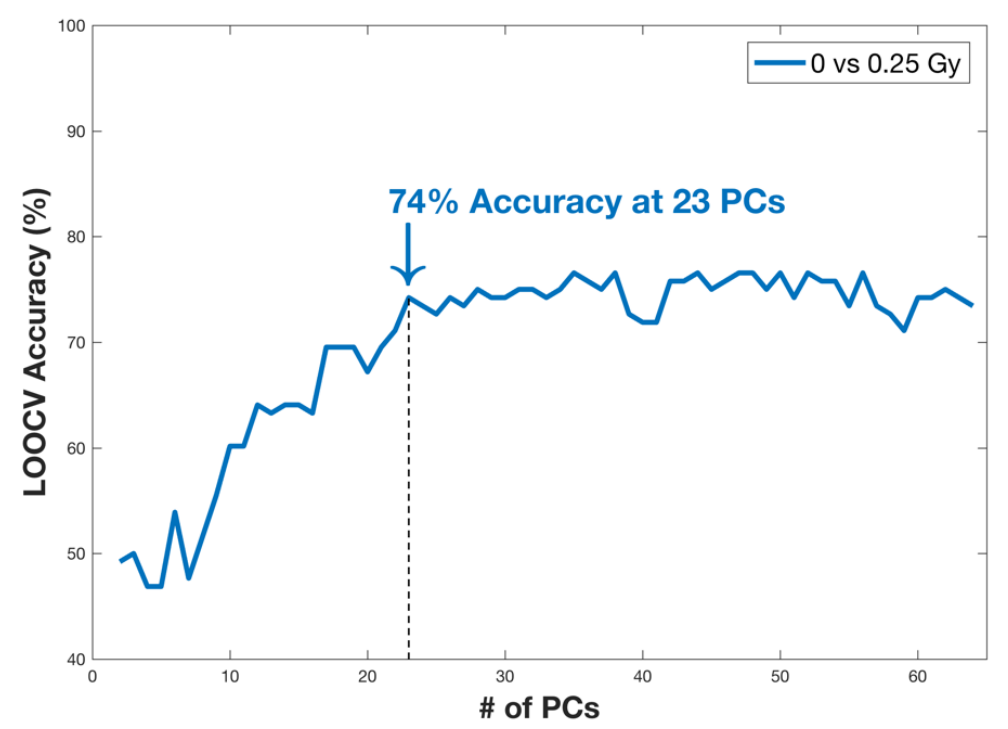

Figure C.8: Plot of LOOCV accuracy vs number of PCs used in the LDA for 0 vs 0.25 Gy, cytoplasm.

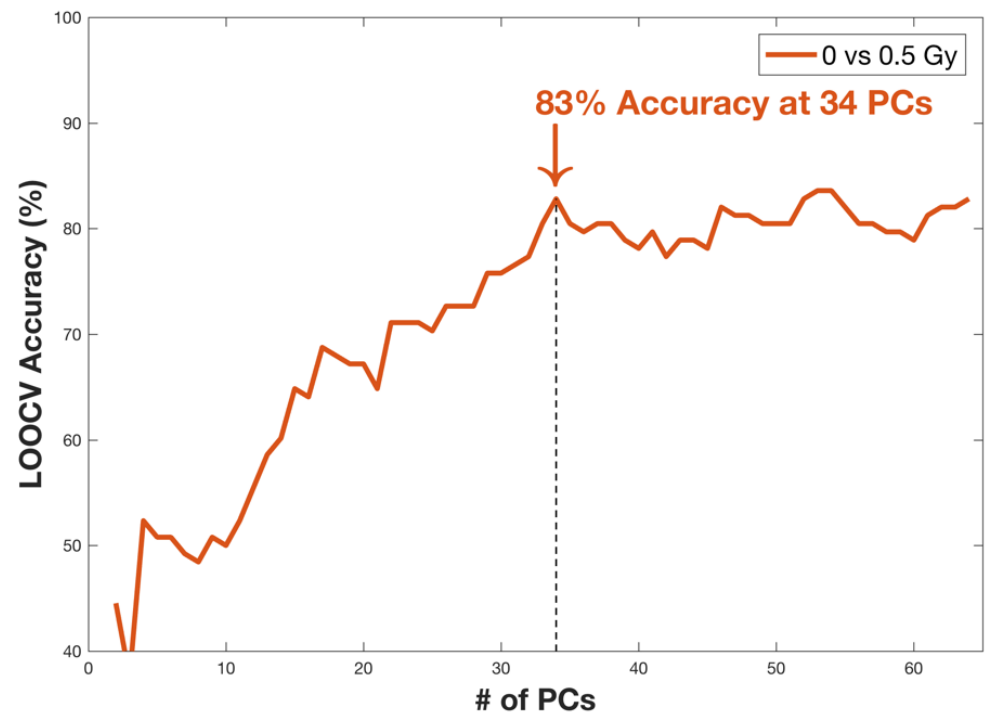

Figure C.9: Plot of LOOCV accuracy vs number of PCs used in the LDA for 0 vs 0.5 Gy, cytoplasm. 


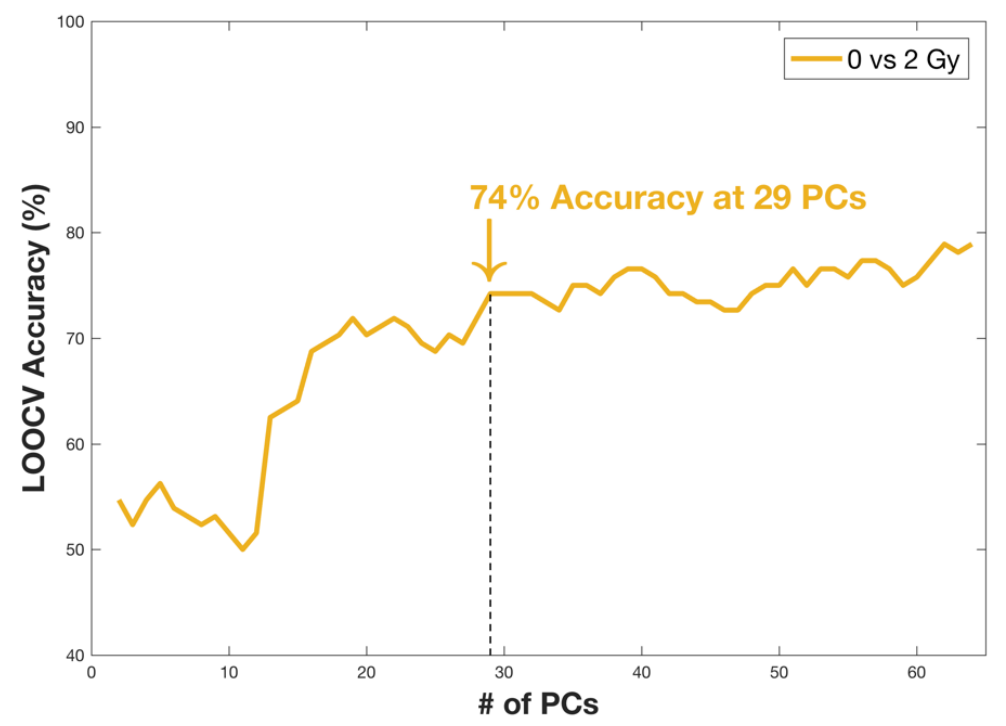

Figure C.10: Plot of LOOCV accuracy vs number of PCs used in the LDA for 0 vs 2 Gy, cytoplasm.

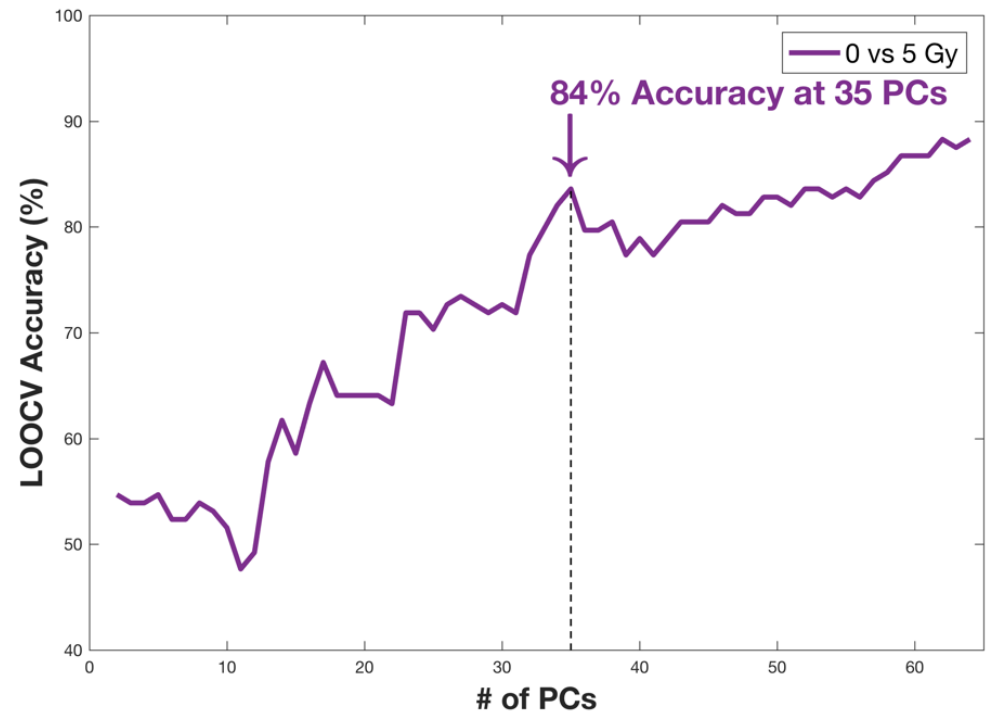

Figure C.11: Plot of LOOCV accuracy vs number of PCs used in the LDA for 0 vs 5 Gy, cytoplasm. 


\section{References}

[1] E. J. Hall and A. J. Giaccia, Radiobiology for the Radiologist, volume 6, Lippincott Williams \& Wilkins Philadelphia, 2006.

[2] E. Vano, L. Gonzalez, J. M. Fernández, and Z. J. Haskal, Eye lens exposure to radiation in interventional suites: caution is warranted, Radiology 248, 945-953 (2008).

[3] S. G. Barnard, E. A. Ainsbury, R. A. Quinlan, and S. D. Bouffler, Radiation protection of the eye lens in medical workers - basis and impact of the ICRP recommendations, The British Journal of Radiology 89, 20151034 (2016).

[4] K. F. Seals, E. W. Lee, C. H. Cagnon, R. A. Al-Hakim, and S. T. Kee, Radiationinduced cataractogenesis: a critical literature review for the interventional radiologist, Cardiovascular and Interventional Radiology 39, 151-160 (2016).

[5] E. A. Ainsbury, S. Barnard, S. Bright, C. Dalke, M. Jarrin, S. Kunze, R. Tanner, J. R. Dynlacht, R. A. Quinlan, and J. Graw, Ionizing radiation induced cataracts: recent biological and mechanistic developments and perspectives for future research, Mutation Research/Reviews in Mutation Research 770, 238-261 (2016).

[6] G. Chodick, N. Bekiroglu, M. Hauptmann, B. H. Alexander, D. M. Freedman, M. M. Doody, L. C. Cheung, S. L. Simon, R. M. Weinstock, and A. Bouville, 
Risk of cataract after exposure to low doses of ionizing radiation: a 20-year prospective cohort study among US radiologic technologists, American journal of epidemiology 168, 620-631 (2008).

[7] E. Vano, R. Sanchez, and J. Fernandez, Estimation of staff lens doses during interventional procedures. Comparing cardiology, neuroradiology and interventional radiology, Radiation protection dosimetry 165, 279-283 (2015).

[8] A. B. Rajabi, F. Noohi, H. Hashemi, M. Haghjoo, M. Miraftab, N. Yaghoobi, F. Rastgou, H. Malek, H. Faghihi, and H. Firouzabadi, Ionizing radiationinduced cataract in interventional cardiology staff, Research in cardiovascular medicine 4 (2015).

[9] K. Kong, C. Kendall, N. Stone, and I. Notingher, Raman spectroscopy for medical diagnostics - From in-vitro biofluid assays to in-vivo cancer detection, Advanced Drug Delivery Reviews 89, 121-134 (2015).

[10] H. J. Butler, L. Ashton, B. Bird, G. Cinque, K. Curtis, J. Dorney, K. EsmondeWhite, N. J. Fullwood, B. Gardner, and P. L. Martin-Hirsch, Using Raman spectroscopy to characterize biological materials, Nature Protocols 11, 664-687 (2016).

[11] M. Jermyn, J. Desroches, K. Aubertin, K. St-Arnaud, W.-J. Madore, E. De Montigny, M.-C. Guiot, D. Trudel, B. C. Wilson, and K. Petrecca, A review of Raman spectroscopy advances with an emphasis on clinical translation challenges in oncology, Physics in Medicine \& Biology 61, R370 (2016).

[12] H. Moradi, A. Ahmad, D. Shepherdson, N. H. Vuong, G. Niedbala, L. Eapen, B. Vanderhyden, B. Nyiri, and S. Murugkar, Raman micro-spectroscopy applied to treatment resistant and sensitive human ovarian cancer cells, Journal of Biophotonics 10, 1327-1334 (2017). 
[13] M. Jermyn, K. Mok, J. Mercier, J. Desroches, J. Pichette, K. Saint-Arnaud, L. Bernstein, M.-C. Guiot, K. Petrecca, and F. Leblond, Intraoperative brain cancer detection with Raman spectroscopy in humans, Science translational medicine 7, 274ra19-274ra19 (2015).

[14] H. Lui, J. Zhao, D. I. McLean, and H. Zeng, Real-time Raman spectroscopy for in vivo skin cancer diagnosis, Cancer Research, canres-4061 (2012).

[15] R. J. Lakshmi, V. Kartha, C. Murali Krishna, J. R. Solomon, G. Ullas, and P. Uma Devi, Tissue Raman spectroscopy for the study of radiation damage: brain irradiation of mice, Radiation Research 157, 175-182 (2002).

[16] A. Synytsya, P. Alexa, J. Besserer, J. De Boer, S. Froschauer, R. Gerlach, M. Loewe, M. Moosburger, I. Obstova, and P. Quicken, Raman spectroscopy of tissue samples irradiated by protons, International Journal of Radiation Biology 80, 581-591 (2004).

[17] Q. Matthews, A. Brolo, J. Lum, X. Duan, and A. Jirasek, Raman spectroscopy of single human tumour cells exposed to ionizing radiation in vitro, Physics in Medicine \& Biology 56, 19 (2010).

[18] Q. Matthews, A. Jirasek, J. Lum, and A. Brolo, Biochemical signatures of in vitro radiation response in human lung, breast and prostate tumour cells observed with Raman spectroscopy, Physics in Medicine \& Biology 56, 6839 (2011).

[19] S. Devpura, K. N. Barton, S. L. Brown, O. Palyvoda, S. Kalkanis, V. M. Naik, F. Siddiqui, R. Naik, and I. J. Chetty, Vision 20/20: the role of Raman spectroscopy in early stage cancer detection and feasibility for application in radiation therapy response assessment, Medical Physics 41 (2014).

[20] M. Yasser, R. Shaikh, M. K. Chilakapati, and T. Teni, Raman spectroscopic 
study of radioresistant oral cancer sublines established by fractionated ionizing radiation, PloS one 9, e97777 (2014).

[21] S. J. Harder, Q. Matthews, M. Isabelle, A. G. Brolo, J. J. Lum, and A. Jirasek, A Raman spectroscopic study of cell response to clinical doses of ionizing radiation, Applied Spectroscopy 69, 193-204 (2015).

[22] Q. Matthews, M. Isabelle, S. J. Harder, J. Smazynski, W. Beckham, A. G. Brolo, A. Jirasek, and J. J. Lum, Radiation-induced glycogen accumulation detected by single cell Raman spectroscopy is associated with radioresistance that can be reversed by metformin, PloS One 10, e0135356 (2015).

[23] A. Maguire, I. Vegacarrascal, L. White, B. McClean, O. Howe, F. Lyng, and A. Meade, Analyses of ionizing radiation effects in vitro in peripheral blood lymphocytes with Raman spectroscopy, Radiation Research 183, 407-416 (2015).

[24] S. J. Harder, M. Isabelle, L. DeVorkin, J. Smazynski, W. Beckham, A. G. Brolo, J. J. Lum, and A. Jirasek, Raman spectroscopy identifies radiation response in human non-small cell lung cancer xenografts, Scientific Reports 6, 21006 (2016).

[25] A. D. Meade, O. Howe, V. Unterreiner, G. D. Sockalingum, H. J. Byrne, and F. M. Lyng, Vibrational spectroscopy in sensing radiobiological effects: analyses of targeted and non-targeted effects in human keratinocytes, Faraday Discussions 187, 213-234 (2016).

[26] K. Sailer, Radiation-induced structural modifications in dsDNA analysed by FT-Raman spectroscopy, International Journal of Radiation Biology 69, 601$613(1996)$.

[27] C. Shaw and A. Jirasek, The use of ultraviolet resonance Raman spectroscopy in the analysis of ionizing-radiation-induced damage in DNA, Applied Spectroscopy 63, 412-422 (2009). 
[28] S. P. Verma, Low levels of irradiation modify lipid domains in model membranes: a laser Raman study, Radiation research 107, 183-193 (1986).

[29] F. H. Attix, Introduction to radiological physics and radiation dosimetry, WileyVCH, Weinheim, Baden-Württemberg, Germany, 2004.

[30] E. B. Podgorsak, Radiation physics for medical physicists, Spring-Verlag, Berlin/Heidelberg, Germany, 2006.

[31] H. E. Johns and J. R. Cunningham, Physics of radiology, Charles C Thomas, Springfield, Illinois, USA, fourth edition, 1983.

[32] M. Berger, J. Hubbell, S. Seltzer, J. Chang, J. Coursey, R. Sukumar, D. Zucker, and K. Olsen, XCOM: Photon Cross Section Database (version 1.5), [Online] Available: https://physics.nist.gov/PhysRefData/Xcom/html/xcom1.html [2018, September 5], 2010, National Institute of Standards and Technology.

[33] G. M. Cooper and R. E. Hausman, The cell: a molecular approach, volume 2, Sinauer associates Sunderland, MA, 2000.

[34] F. Hutchinson, The distance that a radical formed by ionizing radiation can diffuse in a yeast cell, Radiation Research 7, 473-483 (1957).

[35] M. C. Joiner and A. Van der Kogel, Basic clinical radiobiology, Hodder Arnold, London, England, 4 edition, 2009.

[36] R. B. Richardson and M.-E. Harper, Mitochondrial stress controls the radiosensitivity of the oxygen effect: Implications for radiotherapy, Oncotarget 7, 21469 (2016).

[37] Y. E. Dubrova, A. J. Jeffreys, and A. M. Malashenko, Mouse minisatellite mutations induced by ionizing radiation, Nature Genetics 5, 92 (1993). 
[38] Y. E. Dubrova, M. Plumb, B. Gutierrez, E. Boulton, and A. J. Jeffreys, Genome stability: transgenerational mutation by radiation, Nature 405, 37 (2000).

[39] W. P. Roos, A. D. Thomas, and B. Kaina, DNA damage and the balance between survival and death in cancer biology, Nature Reviews Cancer 16, 20 (2016).

[40] M. R. Lieber, The mechanism of double-strand DNA break repair by the nonhomologous DNA end-joining pathway, Annual Review of Biochemistry 79, $181-211(2010)$.

[41] T. T. Paull, E. P. Rogakou, V. Yamazaki, C. U. Kirchgessner, M. Gellert, and W. M. Bonner, A critical role for histone H2AX in recruitment of repair factors to nuclear foci after DNA damage, Current Biology 10, 886-895 (2000).

[42] C. Mothersill and C. Seymour, Cell-cell contact during gamma irradiation is not required to induce a bystander effect in normal human keratinocytes: evidence for release during irradiation of a signal controlling survival into the medium, Radiation Research 149, 256-262 (1998).

[43] K. Chadwick and H. Leenhouts, A molecular theory of cell survival, Physics in Medicine \& Biology 18, 78 (1973).

[44] M. C. Joiner, B. Marples, P. Lambin, S. C. Short, and I. Turesson, Low-dose hypersensitivity: current status and possible mechanisms, International Journal of Radiation Oncology Biology Physics 49, 379-389 (2001).

[45] M. Joiner and H. Johns, Renal damage in the mouse: the response to very small doses per fraction, Radiation Research 114, 385-398 (1988).

[46] N. P. Brown, The lens is more sensitive to radiation than we had believed, 1997.

[47] N. Hamada, Ionizing radiation sensitivity of the ocular lens and its dose rate dependence, International Journal of Radiation Biology 93, 1024-1034 (2017). 
[48] D. A. Long, The Raman effect: a unified treatment of the theory of Raman scattering by molecules, John Wiley and Sons, Chichester, West Sussex, England, 2002.

[49] E. B. Wilson, J. C. Decius, and P. C. Cross, Molecular vibrations: the theory of infrared and Raman vibrational spectra, McGraw-Hill Book Company, New York, USA, 1955.

[50] E. Smith and G. Dent, Modern Raman spectroscopy: a practical approach, John Wiley \& Sons, Chichester, West Sussex, England, 2013.

[51] J. R. Ferraro, Introductory Raman spectroscopy, Academic Press, San Diego, CA, USA, 1994.

[52] J. M. Lerner, Imaging spectrometer fundamentals for researchers in the biosciences - a tutorial, Cytometry Part A: the journal of the International Society for Analytical Cytology 69, 712-734 (2006).

[53] I. R. Lewis and H. Edwards, Handbook of Raman spectroscopy: from the research laboratory to the process line, CRC Press, Boca Raton, 2001.

[54] R. L. McCreery, Raman spectroscopy for chemical analysis, John Wiley \& Sons, New York, NY, USA, 2000.

[55] J. Pawley, Handbook of biological confocal microscopy, Springer Science \& Business Media, Berlin/Heidelberg, Germany, 2010.

[56] R. Gautam, S. Vanga, F. Ariese, and S. Umapathy, Review of multidimensional data processing approaches for Raman and infrared spectroscopy, EPJ Techniques and Instrumentation 2, 8 (2015).

[57] G. Cowan, Statistical data analysis, Oxford University Press, New York, NY, USA, 1998. 
[58] H. Moradi, Development of Raman micro-spectroscopy to characterize human ovarian cancer cells, Master's thesis, Carleton University, 1125 Colonel By Dr, Ottawa, ON, 2016.

[59] I. Notingher, S. Verrier, H. Romanska, A. Bishop, J. Polak, and L. Hench, In situ characterisation of living cells by Raman spectroscopy, Journal of Spectroscopy 16, 43-51 (2002).

[60] B. D. Beier and A. J. Berger, Method for automated background subtraction from Raman spectra containing known contaminants, Analyst 134, 1198-1202 (2009).

[61] S. Orfanidis, Introduction to signal processing, Pearson Education, London, England, 2010.

[62] A. Savitzky and M. J. Golay, Smoothing and differentiation of data by simplified least squares procedures., Analytical chemistry 36, 1627-1639 (1964).

[63] B. L. Welch, The generalization ofstudent's' problem when several different population variances are involved, Biometrika 34, 28-35 (1947).

[64] I. Jolliffe, Principal Component Analysis, Springer, second edition, 2002.

[65] Q. Matthews, A. Jirasek, J. Lum, X. Duan, and A. G. Brolo, Variability in Raman spectra of single human tumor cells cultured in vitro: correlation with cell cycle and culture confluency, Applied Spectroscopy 64, 871-887 (2010).

[66] Z. Movasaghi, S. Rehman, and I. U. Rehman, Raman spectroscopy of biological tissues, Applied Spectroscopy Reviews 42, 493-541 (2007).

[67] J. De Gelder, K. De Gussem, P. Vandenabeele, and L. Moens, Reference database of Raman spectra of biological molecules, Journal of Raman Spectroscopy: An International Journal for Original Work in all Aspects of Raman Spectroscopy, 
Including Higher Order Processes, and also Brillouin and Rayleigh Scattering 38, 1133-1147 (2007).

[68] H. Tang, H. Yao, G. Wang, Y. Wang, Y.-q. Li, and M. Feng, NIR Raman spectroscopic investigation of single mitochondria trapped by optical tweezers, Optics Express 15, 12708-12716 (2007).

[69] S. Borel, E. Prikryl, N. Vuong, J. Jonkman, B. Vanderhyden, B. Wilson, and S. Murugkar, Discrimination of normal and malignant mouse ovarian surface epithelial cells in vitro using Raman microspectroscopy, Analytical Methods 7, 9520-9528 (2015).

[70] V. Sriramoju, A. Alimova, R. Chakraverty, A. Katz, S. Gayen, L. Larsson, H. Savage, and R. Alfano, Raman spectroscopic study of acute oxidative stress induced changes in mice skeletal muscles, in Biomedical Optical Spectroscopy, volume 6853, page 685315, 2008.

[71] N. Uzunbajakava, A. Lenferink, Y. Kraan, B. Willekens, G. Vrensen, J. Greve, and C. Otto, Nonresonant Raman imaging of protein distribution in single human cells, Biopolymers 72, 1-9 (2003).

[72] P. A. Oliver and R. M. Thomson, Investigating energy deposition within cell populations using Monte Carlo simulations, Physics in Medicine \& Biology (2018).

[73] M. Gu, T. Tannous, and C. Sheppard, Improved axial resolution in confocal fluorescence microscopy using annular pupils, Optics Communications 110, 533539 (1994).

[74] P. Caspers, G. Lucassen, H. Bruining, and G. Puppels, Automated depthscanning confocal Raman microspectrometer for rapid in vivo determination of water concentration profiles in human skin, Journal of Raman Spectroscopy 31, $813-818(2000)$. 
[75] S. P. Curram and J. Mingers, Neural networks, decision tree induction and discriminant analysis: An empirical comparison, Journal of the Operational Research Society 45, 440-450 (1994).

[76] Y. Chen and L. Dai, Automated decomposition algorithm for Raman spectra based on a Voigt line profile model, Applied optics 55, 4085-4094 (2016).

[77] V. Vrabie, C. Gobinet, O. Piot, A. Tfayli, P. Bernard, R. Huez, and M. Manfait, Independent component analysis of Raman spectra: application on paraffinembedded skin biopsies, Biomedical Signal Processing and Control 2, 40-50 (2007). 